\title{
A SPECTRAL EM ALGORITHM FOR DYNAMIC FACTOR MODELS
}

Gabriele Fiorentini. Alessandro Galesi and Enrigue Sentana.

Documentos de Trabajo N. 1619

\section{BANCO DE ESPANAA}

Eurosistema

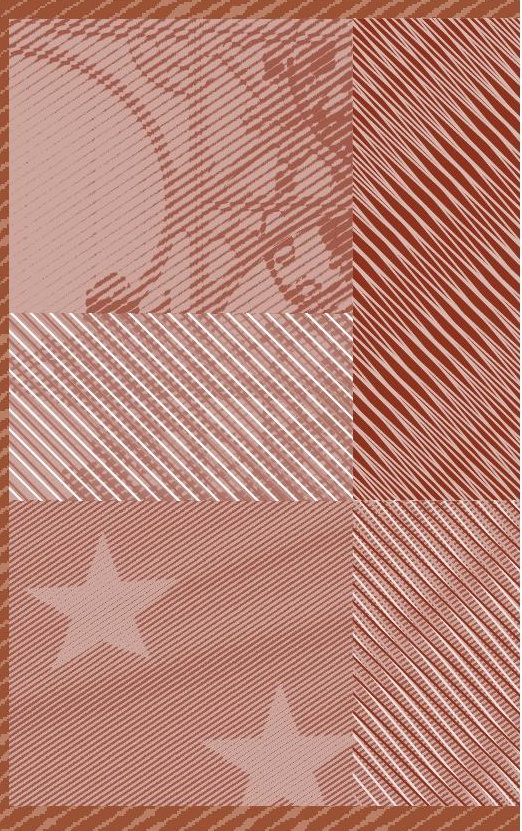


A SPECTRAL EM ALGORITHM FOR DYNAMIC FACTOR MODELS ${ }^{(*)}$

\section{Gabriele Fiorentini}

UNIVERSITÀ DI FIRENZE and RCEA

Alessandro Galesi

BANCO DE ESPAÑA

Enrique Sentana

CEMFI

(*) We are grateful to Dante Amengual and Domenico Giannone, as well as to audiences at the Conference on Indirect Estimation Methods in Finance and Economics (Konstanz, 2014), the Econometric Methods for Banking and Finance Conference (Bank of Portugal, 2014), the NBER-NSF Time Series Conference (Federal Reserve Bank of St. Louis, 2014), the Advances in Econometrics Conference on Dynamic Factor Models (Aarhus, 2014), the EC2 Advances in Forecasting Conference (UPF, 2014), the Italian Congress of Econometrics and Empirical Economics (Salerno, 2015) and the V Workshop in Time Series Econometrics (Zaragoza, 2015) for helpful comments, discussions and suggestions. The requests of the editors and two anonymous referees have also substantially improved the paper. Of course, the usual caveat applies. The views expressed herein are those of the authors and do not necessarily reflect the opinion of the Banco de España or the Eurosystem. Financial support from MIUR through the project "Multivariate statistical models for risk assessment» (Fiorentini) and the Spanish Ministries of Science \& Innovation and Economy \& Competitiveness through grants ECO 2008-00280 and ECO 2014-59262 (Sentana) is also gratefully acknowledged. 
The Working Paper Series seeks to disseminate original research in economics and finance. All papers have been anonymously refereed. By publishing these papers, the Banco de España aims to contribute to economic analysis and, in particular, to knowledge of the Spanish economy and its international environment.

The opinions and analyses in the Working Paper Series are the responsibility of the authors and, therefore, do not necessarily coincide with those of the Banco de España or the Eurosystem.

The Banco de España disseminates its main reports and most of its publications via the Internet at the following website: http://www.bde.es.

Reproduction for educational and non-commercial purposes is permitted provided that the source is acknowledged.

C BANCO DE ESPAÑA, Madrid, 2016

ISSN: 1579-8666 (on line) 


\section{Abstract}

We make two complementary contributions to efficiently estimate dynamic factor models: a frequency domain EM algorithm and a swift iterated indirect inference procedure for ARMA models with no asymptotic efficiency loss for any finite number of iterations. Although our procedures can estimate such models with many series without good initial values, near the optimum we recommend switching to a gradient method that analytically computes spectral scores using the EM principle. We successfully employ our methods to construct an index that captures the common movements of US sectoral employment growth rates, which we compare to the indices obtained by semiparametric methods.

Keywords: indirect inference, Kalman filter, sectoral employment, spectral maximum likelihood, Wiener-Kolmogorov filter.

JEL classification: C32, C38, C51. 


\section{Resumen}

Realizamos dos contribuciones complementarias para estimar eficientemente modelos factoriales dinámicos: un algoritmo EM espectral y un procedimiento de inferencia indirecta iterada rapidísimo para modelos ARMA sin pérdida de eficiencia asintótica para cualquier número finito de iteraciones. Aunque nuestros métodos pueden estimar dichos modelos con muchas series sin buenas condiciones iniciales, cerca del óptimo recomendamos cambiar a un algoritmo del gradiente calculando analíticamente los gradientes espectrales usando el principio EM. Empleamos con éxito nuestros procedimientos para construir un índice que captura los movimientos comunes de las tasas de crecimiento del empleo a escala sectorial en Estados Unidos y lo comparamos con índices obtenidos con métodos semiparamétricos.

Palabras clave: inferencia indirecta, filtro de Kalman, empleo sectorial, máxima verosimilitud espectral, filtro de Wiener-Kolmogorov.

Códigos JEL: C32, C38, C51. 


\section{Introduction}

Dynamic factor models have been extensively used in macroeconomics and finance since their introduction by Geweke (1977) and Sargent and Sims (1977) as a way of capturing the crosssectional and dynamic correlations between multiple series in a parsimonious way. A far from comprehensive list of early and more recent applications include not only business cycle analysis (see Litterman and Sargent (1979), Stock and Watson (1989, 1993), Diebold and Rudebusch (1996) or Gregory, Head and Raynauld (1997)) and bond yields (Singleton (1981), Jegadeesh and Pennacchi (1996), Dungey, Martin and Pagan (2000) or Diebold, Rudebusch and Aruoba (2006)), but also wages (Engle and Watson (1981)), employment (Quah and Sargent (1993)), commodity prices (Peña and Box (1987)) and financial contagion (Mody and Taylor (2007)).

An expanding, influential body of literature has shown that one may accurately recover the unobserved series by using the frequency domain version of principal components put forward by Brillinger (1981, ch. 9) and further extended by Forni, Hallin, Lippi and Reichlin (2000) (FHLR), which is based on a non-parametric estimate of the spectral density matrix of the observed series (see Forni, Hallin, Lippi and Zaffaroni (2015) for more recent developments). In fact, it might even be possible to use static principal components if certain additional assumptions hold (see Bai and $\mathrm{Ng}(2008)$ ). Aside from avoiding the numerical optimisation of a criterion function, the main advantage of such methods is that they remain valid in the presence of some mild contemporaneous and dynamic correlation between idiosyncratic terms when the cross-sectional dimension, $N$, is commensurate with the time series dimension, $T$.

There are two closely related issues, though. First, the cross-sectional asymptotic boundedness conditions on the eigenvalues of the autocovariance matrices of the idiosyncratic terms underlying the approximate factor structures originally suggested by Chamberlain and Rothschild (1983) are largely meaningless in empirical situations in which $N$ is small relative to $T$. And second, although the factors could be regarded as a set of parameters in any given realisation, efficiency considerations indicate that a signal extraction approach which treats the underlying latent variables as stochastic processes would be more appropriate for such data sets. In addition, Doz, Giannone and Reichlin (2012) have recently closed the gap between the two strands of the literature by proving the NT-consistency of the Gaussian pseudo ML estimators (MLE) of exact versions of dynamic factor models even when not all the maintained assumptions hold.

In principle, Gaussian (P)MLEs of the parameters can be obtained from the usual time domain version of the log-likelihood function computed as a by-product of the Kalman filter prediction equations or from Whittle's (1962) frequency domain asymptotic approximation. Further, 
once the parameters have been estimated the Kalman smoother or its Wiener-Kolmogorov counterpart provide optimally filtered estimates of the latent factors. These estimation and filtering issues are well understood (see e.g. Harvey (1989)), and the same can be said of their numerical implementation (see Jungbacker and Koopman (2015)). In practice, though, researchers avoid ML except in relatively small models because of the heavy computational burden involved, which is disproportionately larger as the number of series considered increases.

To ameliorate this problem, Shumway and Stoffer (1982), Watson and Engle (1983) and Quah and Sargent (1993) applied the Expectation-Maximisation (EM) algorithm in Dempster, Laird and Rubin (1977) to the time domain versions of these models, thereby avoiding the computation of the likelihood function and its score. This iterative algorithm has been popular in various areas of statistics and econometrics when the data set is incomplete or contains missing values, or the model can be posed in a similar form, such as in the finite mixture models studied by Arcidiacono and Jones (2003) or the dynamic Markov switching models considered by Hamilton (1990) and Fruhwirth-Schnatter (2007). Its popularity can be attributed mainly to the efficiency of the procedure, as measured by its speed, and also to the generality of the approach and its convergence properties (see Ruud (1991) for an elegant review of this method and McLachlan and Krishnan (1996) for a more thorough analysis).

However, the time domain version of the EM algorithm has only been derived for dynamic factor models in which the latent variables follow pure AR processes (see again Doz, Giannone and Reichlin (2012)), and works best when the effects of the common factors on the observed variables are contemporaneous, which substantially limits the class of models to which they can be successfully applied. In particular, it excludes models in which either common or idiosyncratic factors follow ARMA processes. As is well known, such processes combine autoregressive and moving average components in a rather parsimonious way, and for that reason they are by far the most common approximations used to (Wold) represent univariate stationary series. However, while AR process often arise as difference equation-type representations of natural phenomena, the presence of MA components is sometimes justified as a result of contemporaneous aggregation of several underlying components. On this basis, one might argue that there is no need to introduce MA terms in dynamic factor models.

Nevertheless, there are at least three compelling reasons for considering ARMA processes for common or idiosyncratic factors. First, the temporal aggregation results in Bergstrom (1984) imply that discrete time observations will often contain MA components even if the underlying continuous time processes follow first-order stochastic difference equations. Obviously, the same applies to the increasingly popular continuous time versions of ARMA models (see Chambers and Thorton (2012)). Second, the usual deseasonalisation procedures employed by the national 
statistical offices imply a transfer function that substantially dampens the spectral density at high frequencies, which pure AR models struggle to capture (see Maravall (1993)). Given that those filters tend to be very similar for closely related homogeneous series, they are likely to introduce MA terms not only in the specific component of each series but also in the common one. Finally, recent macroeconomic applications of dynamic factor models have often considered specifications in which the lagged latent variables appear as additional factors (see again Bai and $\mathrm{Ng}$ (2008) and the references therein). In those circumstances, plausible cross-sectional restrictions on the dynamic factor loadings made for parsimony reasons also introduce MA terms in the common factors.

In the context of general dynamic factor models with latent ARMA processes, we make two independent but complementary contributions. First, we introduce a frequency domain version of the EM algorithm, which exploits the heteroskedastic factor structure of the spectral density matrix of the model to carry out the expectation stage very quickly. Nevertheless, a standard implementation of our algorithm would still require $O(N)$ numerical optimisations at each maximisation stage in models with idiosyncratic MA components. For that reason, our second contribution is a very fast iterated indirect inference procedure for estimating the parameters of univariate ARMA models, which is based on a sequence of simple auxiliary OLS regressions of certain filtered series. Importantly, unlike existing indirect inference procedures for those models, our proposed estimator entails no asymptotic efficiency loss for any finite number of iterations. Further, it will generally coincide with the ML estimator in the limit.

The complementary between our proposals is twofold: (i) our iterated indirect inference method can be implemented far more quickly in the frequency domain than in the time domain; and (ii) it can be very easily adapted to deal with latent variables with only minor modifications.

Our combined proposal, though, differs from more standard applications of indirect estimation methods to factor models, which typically rely on a simpler approximating model as auxiliary model. For example, in the case of a single factor with static loadings we could fit univariate ARMA models to the first principal component of the observed series, as well as to the difference between each of the observed series and its common component. Such procedures will result in an efficiency loss relative to maximum likelihood without yielding any gains in terms of speed because the calibration of the parameters of the true model by minimising a GMM-type criterion function whose moments have to be evaluated by simulation would remain prohibitive (but see Sentana, Calzolari and Fiorentini (2008) for a sequential proposal that could speed up the indirect estimation of large models).

Finally, we illustrate our proposed procedures with an empirical application to US employment data. Specifically, we follow Quah and Sargent (1993) and construct an index that captures 
the common movements of sectoral employment growth rates. Nevertheless, we also compare our results to the ones obtained by the aforementioned semiparametric approaches.

The rest of the paper is organised as follows. In section 2, we review the properties of dynamic factor models and their filters, as well as maximum likelihood estimation in the frequency domain. Then, we derive our iterated indirect procedure in section 3 and our spectral EM algorithm in section 4 . This is followed by the empirical application in section 5 . Finally, we discuss several interesting extensions for further research in section 6. Auxiliary results are gathered in appendices.

\section{Theoretical background}

\subsection{Dynamic factor models}

To keep the notation to a minimum, we focus on single factor models, which suffice to illustrate our procedures. A dynamic, exact, single factor model for a finite dimensional vector of $N$ observed series, $\mathbf{y}_{t}$, can be defined in the time domain by the system of stochastic difference equations

$$
\left.\begin{array}{c}
\mathbf{y}_{t}=\boldsymbol{\mu}+\mathbf{c}(L) x_{t}+\mathbf{u}_{t}, \\
\alpha_{x}(L) x_{t}=\beta_{x}(L) f_{t}, \\
\left(f_{t}, v_{1, t}, \ldots, v_{N, t}\right) \mid I_{t-1} ; \boldsymbol{\mu}, \boldsymbol{\theta} \sim N\left[0, \operatorname{diag}\left(\psi_{f}, \psi_{1}, \ldots, \psi_{N}\right)\right],
\end{array}\right\}
$$

where $x_{t}$ is the only common factor, $\mathbf{u}_{t}$ the $N$ specific factors, $\mathbf{c}(L)=\sum_{k=-m}^{n} \mathbf{c}_{k} L^{k}$ a vector of $N$ possibly two-sided polynomials in the lag operator $c_{i}(L), \alpha_{x}(L)$ and $\alpha_{u_{i}}(L)$ are one-sided polynomials of orders $p_{x}$ and $p_{u_{i}}$, respectively, while $\beta_{x}(L)$ and $\beta_{u_{i}}(L)$ are one-sided polynomials of orders $q_{x}$ and $q_{u_{i}}$ coprime with $\alpha_{x}(L)$ and $\alpha_{u_{i}}(L)$, respectively, $I_{t-1}$ is an information set that contains the values of $\mathbf{v}_{t}$ and $f_{t}$ up to, and including time $t-1, \boldsymbol{\mu}$ is the mean vector and $\boldsymbol{\theta}$ refers to all the remaining model parameters, which we assume variation-free.

Note that the dynamic nature of the model is the result of three different characteristics:

1. The serial correlation of the common factor $x_{t}$,

2. The serial correlation of the idiosyncratic factors $\mathbf{u}_{t}$ and

3. The heterogeneous dynamic impact of the common factor on each of the observed variables through the series-specific distributed lag polynomials $c_{i}(L)$.

To some extent, characteristics 1 and 3 overlap, as one could always write any dynamic factor model in terms of white noise common factors. In this regard, the assumption of $\operatorname{Arma}\left(p_{x}, q_{x}\right)$ dynamics for the common factor can be regarded as a parsimonious way of modelling an infinite distributed lag (see sections 2.3 and 4.5 for further details). In any case, we would need to shut 
down all three sources to go back to a traditional static factor model (see Lawley and Maxwell (1971)). Cancelling only one or two of those channels still results in a dynamic factor model. For example, Engle and Watson (1981) considered models with static factor loadings, while Peña and Box (1987) further assumed that the specific factors were white noise.

The main difference between the exact model in (1) and the generalised dynamic factor models considered by Forni, Hallin, Lippi and Reichlin (2000), Forni and Lippi (2001, 2011) and Forni, Hallin, Lippi and Zaffaroni (2015) is that it rules out any contemporaneous or dynamic cross-correlation between the idiosyncratic terms. We revisit this issue in section 6 .

\subsection{Spectral density matrix and filters}

Under the assumption that $\mathbf{y}_{t}$ is a covariance stationary process, possibly after suitable transformations as in section 5 , its spectral decomposition will be

$$
\begin{aligned}
\mathbf{y}_{t}-\boldsymbol{\mu} & =\int_{-\pi}^{\pi} e^{i \lambda t} d \mathbf{Z}^{\mathbf{y}}(\lambda), \\
V\left[d \mathbf{Z}^{\mathbf{y}}(\lambda)\right] & =\mathbf{G}_{\mathbf{y y}}(\lambda) d \lambda,
\end{aligned}
$$

with a spectral density matrix given by

$$
\begin{gathered}
\mathbf{G}_{\mathbf{y y}}(\lambda)=\mathbf{c}\left(e^{-i \lambda}\right) G_{x x}(\lambda) \mathbf{c}^{\prime}\left(e^{i \lambda}\right)+\mathbf{G}_{\mathbf{u u}}(\lambda), \\
G_{x x}(\lambda)=\frac{\beta_{x}\left(e^{-i \lambda}\right) \beta_{x}\left(e^{i \lambda}\right)}{\alpha_{x}\left(e^{-i \lambda}\right) \alpha_{x}\left(e^{i \lambda}\right)} \psi_{f} \\
\mathbf{G}_{\mathbf{u u}}(\lambda)=\operatorname{diag}\left[G_{u_{1} u_{1}}(\lambda), \ldots, G_{u_{N} u_{N}}(\lambda)\right] \\
G_{u_{i} u_{i}}(\lambda)=\frac{\beta_{u_{i}}\left(e^{-i \lambda}\right) \beta_{u_{i}}\left(e^{i \lambda}\right)}{\alpha_{u_{i}}\left(e^{-i \lambda}\right) \alpha_{u_{i}}\left(e^{i \lambda}\right)} \psi_{i} .
\end{gathered}
$$

Thus, (1) implies that $\mathbf{G}_{\mathbf{y y}}(\lambda)$ is the sum of the rank 1 matrix $\mathbf{c}\left(e^{-i \lambda}\right) G_{x x}(\lambda) \mathbf{c}^{\prime}\left(e^{i \lambda}\right)$ and the diagonal matrix $\mathbf{G}_{\mathbf{u u}}(\lambda)$, thereby inheriting the exact single factor structure of the unconditional covariance matrix of a static factor model.

The fact that the idiosyncratic impact of the common factor on each of the observed variables is in principle dynamic implies that the spectral density matrix of $\mathbf{y}_{t}$ will generally be complex but Hermitian, even though the spectral densities of $x_{t}$ and $u_{i t}$ are all real because they correspond to univariate processes.

Assuming that $\mathbf{G}_{\mathbf{y y}}(\lambda)$ is not singular at any frequency, the Wiener-Kolmogorov two-sided filter for the common factor $x_{t}$ at each frequency is given by

$$
d Z^{x^{K}}(\lambda)=G_{x x}(\lambda) \mathbf{c}^{\prime}\left(e^{i \lambda}\right) \mathbf{G}_{\mathbf{y y}}^{-1}(\lambda) d \mathbf{Z}^{\mathbf{y}}(\lambda)
$$


where

$$
G_{x x}(\lambda) \mathbf{c}^{\prime}\left(e^{i \lambda}\right) \mathbf{G}_{\mathbf{y y}}^{-1}(\lambda)
$$

is known as the transfer function of the common factor smoother. As a result, the spectral density of the smoothed values of the common factors, $x_{t \mid \infty}^{K},{ }^{1}$ will be

$$
G_{x^{K} x^{K}}(\lambda)=G_{x x}^{2}(\lambda) \mathbf{c}^{\prime}\left(e^{i \lambda}\right) \mathbf{G}_{\mathbf{y y}}^{-1}(\lambda) \mathbf{c}\left(e^{-i \lambda}\right)
$$

thanks to the Hermitian nature of $\mathbf{G}_{\mathbf{y y}}(\lambda)$, while the spectral density of the final estimation error $x_{t}-x_{t \mid \infty}^{K}$ will be given by

$$
G_{x x}(\lambda)-G_{x x}^{2}(\lambda) \mathbf{c}^{\prime}\left(e^{i \lambda}\right) \mathbf{G}_{\mathbf{y y}}^{-1}(\lambda) \mathbf{c}\left(e^{-i \lambda}\right)=\omega(\lambda) .
$$

Similarly, the Wiener-Kolmogorov smoother for the $N$ specific factors will be

$$
\begin{gathered}
d \mathbf{Z}^{\mathbf{u}^{K}}(\lambda)=\mathbf{G}_{\mathbf{u u}}(\lambda) \mathbf{G}_{\mathbf{y y}}^{-1}(\lambda) d \mathbf{Z}^{\mathbf{y}}(\lambda) \\
=\left[\mathbf{I}_{N}-\mathbf{c}\left(e^{-i \lambda}\right) G_{x x}(\lambda) \mathbf{c}^{\prime}\left(e^{i \lambda}\right) \mathbf{G}_{\mathbf{y y}}^{-1}(\lambda)\right] d \mathbf{Z}^{\mathbf{y}}(\lambda)=d \mathbf{Z}^{\mathbf{y}}(\lambda)-\mathbf{c}\left(e^{-i \lambda}\right) d Z^{x^{K}}(\lambda) .
\end{gathered}
$$

Hence, the spectral density matrix of the smoothed values of the specific factors will be given by

$$
\mathbf{G}_{\mathbf{u}^{K} \mathbf{u}^{K}}(\lambda)=\mathbf{G}_{\mathbf{u u}}(\lambda) \mathbf{G}_{\mathbf{y y}}^{-1}(\lambda) \mathbf{G}_{\mathbf{u u}}(\lambda),
$$

while the spectral density of their final estimation errors $\mathbf{u}_{t}-\mathbf{u}_{t \mid \infty}^{K}$ is

$$
\mathbf{G}_{\mathbf{u u}}(\lambda)-\mathbf{G}_{\mathbf{u}^{K} \mathbf{u}^{K}}(\lambda)=\mathbf{G}_{\mathbf{u u}}(\lambda)-\mathbf{G}_{\mathbf{u u}}(\lambda) \mathbf{G}_{\mathbf{y y}}^{-1}(\lambda) \mathbf{G}_{\mathbf{u u}}(\lambda)=\omega(\lambda) \mathbf{c}\left(e^{-i \lambda}\right) \mathbf{c}^{\prime}\left(e^{i \lambda}\right)=\boldsymbol{\Xi}(\lambda) .
$$

Finally, the co-spectrum between $x_{t \mid \infty}^{K}$ and $\mathbf{u}_{t \mid \infty}^{K}$ will be

$$
\mathbf{G}_{x^{K} \mathbf{u}^{K}}(\lambda)=G_{x x}(\lambda) \mathbf{c}^{\prime}\left(e^{i \lambda}\right) \mathbf{G}_{\mathbf{y y}}^{-1}(\lambda) \mathbf{G}_{\mathbf{u u}}(\lambda)
$$

\section{$2.3 \quad$ Identification}

The identification by means of homogeneous restrictions of linear dynamic models with latent variables such as (1) was discussed by Geweke (1977) and Geweke and Singleton (1981), and more recently by Scherrer and Deistler (1998) and Heaton and Solo (2004) (see also Forni and Lippi (2001, 2011) for related results). These authors extend well known results from static factor models and simultaneous equation systems to the spectral density matrix (2) on a frequency by frequency basis. Thus, two models will be observationally equivalent if and only if they generate exactly the same spectral density matrix for the observed variables at all frequencies. As in the traditional case, there are two different identification issues:

\footnotetext{
${ }^{1}$ The main difference between the Wiener-Kolmogorov filtered values, $x_{t \mid \infty}^{K}$, and the Kalman filter smoothed values, $x_{t \mid T}^{K}$, results from the dependence of the former on a double infinite sequence of observations.
} 
1. the nonparametric identification of common and specific components,

2. the parametric identification of dynamic loadings and factor dynamics within the common components.

The answer to the first question is easy when $\mathbf{G}_{\mathbf{u u}}(\lambda)$ is a diagonal, full rank matrix. ${ }^{2}$ Specifically, we can show that for the dynamic single factor model (1), nonparametric identification of common and idiosyncratic terms is guaranteed when $N \geq 3$ provided that at least three series load on the common factor. The intuition is as follows. We know that the condition above coincides with the so-called Ledermann bound for single factor models (see e.g. Scherrer and Deistler (1998)). Since it is not possible to transfer variance from the common to the idiosyncratic components (or vice versa) in those circumstances, and any model with more than one factor will lead to some singular idiosyncratic variance, we can uniquely decompose $\mathbf{G}_{\mathbf{y y}}(\lambda)$ into the rank one matrix $\mathbf{c}\left(e^{-i \lambda}\right) G_{x x}(\lambda) \mathbf{c}^{\prime}\left(e^{i \lambda}\right)$ and the full rank matrix $\mathbf{G}_{\mathbf{u u}}(\lambda)$ in this way.

The separate identification of $\mathbf{c}\left(e^{-i \lambda}\right)$ and $G_{x x}(\lambda)$ is trickier, as we could always write any dynamic factor model (up to time shifts) in terms of white noise common factors. But it can be guaranteed (up to scaling and sign changes) if in addition the dynamic loading polynomials $c_{i}($.$) are one-sided of finite order and coprime, so they do not share a common root across all N$ series (see theorem 3 in Heaton and Solo (2004) for a more formal argument along these lines). ${ }^{3}$

To avoid dealing with nonsensical situations, henceforth we maintain the assumption that the model that has to be estimated is identified, which will have to be verified on a case by case basis. Nevertheless, the conditions above suffice to guarantee identification in the empirical application in section 5. The only remaining issue is the unconditional scaling of the common factor, which we can achieve by normalising the variance of $f_{t}$ to $1{ }^{4}$

\subsection{Maximum likelihood estimation in the frequency domain}

Let

$$
\mathbf{I}_{\mathbf{y y}}\left(\lambda_{j}\right)=\frac{1}{2 \pi T} \sum_{t=1}^{T} \sum_{s=1}^{T}\left(\mathbf{y}_{t}-\boldsymbol{\mu}\right)\left(\mathbf{y}_{s}-\boldsymbol{\mu}\right)^{\prime} e^{-i(t-s) \lambda_{j}}
$$

denote the Hermitian positive semidefinite periodogram matrix of $\mathbf{y}_{t}$ and $\lambda_{j}=2 \pi j / T(j=$ $0, \ldots T-1)$ the usual Fourier frequencies. If we assume that $\mathbf{G}_{\mathbf{y y}}(\lambda)$ is not singular at any of

\footnotetext{
${ }^{2}$ Scherrer and Deistler (1998) refer to this situation as the Frisch case.

${ }^{3}$ The one-sided restriction is without loss of generality in finite order models because any shift in the dating of the common factor can be exactly matched by an opposite shift in the timing of the dynamic loadings.

${ }^{4}$ Other symmetric scaling assumptions would normalise the unconditional variance of $x_{t}$, or some norm of the vector of loadings $\mathbf{c}_{0}$ or their long run counterparts $\mathbf{c}(1)$. Alternatively, we could asymmetrically fix one element of $\mathbf{c}_{0}$ or $\mathbf{c}(1)$ to 1 .
} 
those frequencies, the so-called Whittle (discrete) spectral approximation to the log-likelihood function is ${ }^{5}$

$$
-\frac{N T}{2} \ln (2 \pi)-\frac{1}{2} \sum_{j=0}^{T-1} \ln \left|\mathbf{G}_{\mathbf{y y}}\left(\lambda_{j}\right)\right|-\frac{1}{2} \sum_{j=0}^{T-1} \operatorname{tr}\left\{\mathbf{G}_{\mathbf{y y}}^{-1}\left(\lambda_{j}\right)\left[2 \pi \mathbf{I}_{\mathbf{y y}}\left(\lambda_{j}\right)\right]\right\} .
$$

Expression (4), though, is far from ideal from a computational point of view, and for that reason we make use of the Fast Fourier Transform (FFT). Specifically, given the $T \times N$ original real data matrix $\mathbf{Y}=\left(\mathbf{y}_{1}, \ldots, \mathbf{y}_{t}, \ldots, \mathbf{y}_{T}\right)^{\prime}$, the FFT creates the centred and orthogonalised $T \times N$ complex data matrix $\mathbf{Z}^{\mathbf{y}}=\left(\mathbf{z}_{0}^{\mathbf{y}}, \ldots, \mathbf{z}_{j}^{\mathbf{y}}, \ldots, \mathbf{z}_{T-1}^{\mathbf{y}}\right)^{\prime}$ by effectively premultiplying $\mathbf{Y}-\ell_{T} \boldsymbol{\mu}^{\prime}$ by the $T \times T$ Fourier matrix $\mathbf{W}$. On this basis, we can easily compute $\mathbf{I}_{\mathbf{y y}}\left(\lambda_{j}\right)$ as $2 \pi \mathbf{z}_{j}^{\mathbf{y}} \mathbf{z}_{j}^{\mathbf{y} *}$, where $\mathbf{z}_{j}^{\mathbf{y} *}$ is the complex conjugate transpose of $\mathbf{z}_{j}^{\mathbf{y}}$. Hence, the spectral approximation to the log-likelihood function (5) becomes

$$
-\frac{N T}{2} \ln (2 \pi)-\frac{1}{2} \sum_{j=0}^{T-1} \ln \left|\mathbf{G}_{\mathbf{y y}}\left(\lambda_{j}\right)\right|-\frac{2 \pi}{2} \sum_{j=0}^{T-1} \mathbf{z}_{j}^{\mathbf{y} *} \mathbf{G}_{\mathbf{y y}}^{-1}\left(\lambda_{j}\right) \mathbf{z}_{j}^{\mathbf{y}},
$$

which can be regarded as the log-likelihood function of $T$ independent but heteroskedastic complex Gaussian observations.

But since $\mathbf{z}_{j}^{\mathbf{y}}$ does not depend on $\boldsymbol{\mu}$ for $j=1, \ldots, T-1$ because $\ell_{T}$ is proportional to the first column of the orthogonal Fourier matrix and $\mathbf{z}_{0}^{\mathbf{y}}=\left(\overline{\mathbf{y}}_{T}-\boldsymbol{\mu}\right)$, where $\overline{\mathbf{y}}_{T}$ is the sample mean of $\mathbf{y}_{t}$, it immediately follows that the ML of $\boldsymbol{\mu}$ will be $\overline{\mathbf{y}}_{T}$. As for the remaining parameters, the score function will be given by:

$$
\begin{gathered}
\mathbf{d}(\boldsymbol{\theta})=\frac{1}{2} \sum_{j=0}^{T-1} \mathbf{d}\left(\lambda_{j} ; \boldsymbol{\theta}\right), \\
\mathbf{d}\left(\lambda_{j} ; \boldsymbol{\theta}\right)=\frac{1}{2} \frac{\partial v e c^{\prime}\left[\mathbf{G}_{\mathbf{y y}}\left(\lambda_{j}\right)\right]}{\partial \boldsymbol{\theta}}\left[\mathbf{G}_{\mathbf{y y}}^{-1}\left(\lambda_{j}\right) \otimes \mathbf{G}_{\mathbf{y y}}^{\prime-1}\left(\lambda_{j}\right)\right] v e c\left[2 \pi \mathbf{z}_{j}^{\mathbf{y} c} \mathbf{z}_{j}^{\mathbf{y} \prime}-\mathbf{G}_{\mathbf{y y}}^{\prime}\left(\lambda_{j}\right)\right] \\
=\frac{1}{2} \frac{\partial v e c^{\prime}\left[\mathbf{G}_{\mathbf{y y}}\left(\lambda_{j}\right)\right]}{\partial \boldsymbol{\theta}} \mathbf{M}\left(\lambda_{j}\right) \mathbf{m}\left(\lambda_{j}\right),
\end{gathered}
$$

where $\mathbf{z}_{j}^{\mathbf{y} c}=\mathbf{z}_{j}^{\mathbf{y} * \prime}$ is the complex conjugate of $\mathbf{z}_{j}^{\mathbf{y}}$,

$$
\mathbf{m}\left(\lambda_{j}\right)=\operatorname{vec}\left[2 \pi \mathbf{z}_{j}^{\mathbf{y} c} \mathbf{z}_{j}^{\mathbf{y} \prime}-\mathbf{G}_{\mathbf{y y}}^{\prime}\left(\lambda_{j}\right)\right]
$$

and

$$
\mathbf{M}\left(\lambda_{j}\right)=\mathbf{G}_{\mathbf{y y}}^{-1}\left(\lambda_{j}\right) \otimes \mathbf{G}_{\mathbf{y y}}^{\prime-1}\left(\lambda_{j}\right) .
$$

The information matrix is block diagonal between $\boldsymbol{\mu}$ and the elements of $\boldsymbol{\theta}$, with the $(1,1)$ element being $\mathbf{G}_{\mathbf{y y}}(0)$ and the $(2,2)$-block being

$$
\mathbf{Q}=\frac{1}{4 \pi} \int_{-\pi}^{\pi} \frac{\partial v e c^{\prime}\left[\mathbf{G}_{\mathbf{y y}}(\lambda)\right]}{\partial \boldsymbol{\theta}} \mathbf{M}(\lambda)\left\{\frac{\partial v e c^{\prime}\left[\mathbf{G}_{\mathbf{y y}}(\lambda)\right]}{\partial \boldsymbol{\theta}}\right\}^{*} d \lambda,
$$

\footnotetext{
${ }^{5}$ There is also a continuous version which replaces sums by integrals (see Dunsmuir and Hannan (1976)).
} 
a consistent estimator of which will be provided by either by the outer product of the score or by

$$
\boldsymbol{\Phi}(\boldsymbol{\theta})=\frac{1}{2} \sum_{j=0}^{T-1} \frac{\partial v e c^{\prime}\left[\mathbf{G}_{\mathbf{y y}}\left(\lambda_{j}\right)\right]}{\partial \boldsymbol{\theta}} \mathbf{M}\left(\lambda_{j}\right)\left\{\frac{\partial v e c^{\prime}\left[\mathbf{G}_{\mathbf{y y}}\left(\lambda_{j}\right)\right]}{\partial \boldsymbol{\theta}}\right\}^{*} .
$$

Formal results showing the strong consistency and asymptotic normality of the resulting ML estimators of dynamic latent variable models under suitable regularity conditions were provided by Dunsmuir (1979), who generalised earlier results for VARMa models by Dunsmuir and Hannan (1976). These authors also showed the asymptotic equivalence between time and frequency domain ML estimators. ${ }^{6}$ In addition, they explicitly acknowledged the possibility that the normality assumption does not hold, in which case the criterion function (5) must be understood as a pseudo log-likelihood. ${ }^{7}$

Appendix C provides detailed expressions for the Jacobian of vec $\left[\mathbf{G}_{\mathbf{y y}}(\lambda)\right]$ and the spectral score of dynamic factor models, while appendix E includes numerically reliable and efficient formulae for the information matrix. Those expressions make extensive use of the complex version of the Woodbury formula in appendix A.

Nevertheless, when $N$ is large the number of parameters is huge, and the direct maximisation of the log-likelihood function becomes excruciatingly slow, especially without good initial values. For that reason, in section 4 we derive a much faster alternative to obtain MLEs of all the model parameters based on the EM algorithm. But first, we propose a very fast iterated indirect inference procedure asymptotically equivalent to ML for estimating the parameters of univariate ARMA models, which will provide a very useful complement to the EM algorithm.

\section{Indirect inference estimation of ARMA models}

\subsection{Pure MA terms}

Consider the following MA(1) model

$$
x_{t}=f_{t}-\beta f_{t-1}, \quad|\beta|<1, \quad f_{t} \mid x_{t-1}, x_{t-2}, \ldots \sim N(0,1)
$$

The simplest consistent estimator of $\beta$ is an indirect inference (II) one based on the misspecified Ar(1) auxiliary model

$$
x_{t}=\rho x_{t-1}+\varepsilon_{t}, \quad \varepsilon_{t} \mid x_{t-1}, x_{t-2}, \ldots \sim N(0,1)
$$

\footnotetext{
${ }^{6}$ This equivalence is not surprising in view of the contiguity of the Whittle measure in the Gaussian case (see Choudhuri, Ghosal and Roy (2004)).

${ }^{7}$ See also Quah and Sargent (1993) for a least squares projection interpretation of the EM algorithm under non-normality
} 
(see e.g. Gouriéroux, Monfort and Renault (1993), Chumacero (2001) or Ghysels, Khalaf and Vodounou (2003)). This estimator is equivalent to the GMM estimator of $\beta$ based on

$$
E\left[m_{t}(\beta) \mid \beta\right]=0, m_{t}(\beta)=\left(x_{t}+\frac{\beta}{1+\beta^{2}} x_{t-1}\right) x_{t-1},
$$

which coincides with the score of the $\operatorname{AR}(1)$ parameter $\rho$ evaluated at the binding function

$$
\rho(\beta)=-\frac{\beta}{1+\beta^{2}} .
$$

We could increase the efficiency with which we estimate $\beta$ by II if we considered higher order $\operatorname{AR}(\mathrm{k})$ models for $k \geq 2$. Unfortunately, for any finite order $k$ those II estimators of $\beta$ are generally inefficient relative to the ML estimator, which is effectively based on the moment condition

$$
E\left[s_{t}(\beta) \mid \beta\right]=0, \quad s_{t}(\beta)=\left[x_{t}-\nu_{t}(\beta)\right] \frac{\partial \nu_{t}(\beta)}{\partial \beta},
$$

where

$$
\nu_{t}(\beta)=E\left(x_{t} \mid x_{t-1}, x_{t-2}, \ldots ; \beta\right)=-\sum_{j=1}^{\infty} \beta^{j} x_{t-j}=-\frac{\beta L}{1-\beta L} x_{t}
$$

is the conditional mean of $x_{t}$ given its past under the maintained assumption that the MA(1) process is invertible.

At first sight, it would appear that this highly non-linear estimator cannot be obtained by applying OLS to some auxiliary linear autoregressive model, but appearances can sometimes be misleading. Define

$$
f_{t}(\beta)=x_{t}-\nu_{t}(\beta)=\sum_{j=0}^{\infty} \beta^{j} x_{t-j}=\frac{1}{1-\beta L} x_{t}
$$

as the "innovations" in $x_{t}$. Similarly, let us use the shorthand notation

$$
w_{t}(\beta)=-\frac{\partial \nu_{t+1}(\beta)}{\partial \beta}=\sum_{j=0}^{\infty}(j+1) \beta^{j} x_{t-j}=\frac{1}{(1-\beta L)^{2}} x_{t} .
$$

We know that at the true value of $\beta$, say $\beta_{0}, f_{t}\left(\beta_{0}\right)$ will be white noise while $w_{t}\left(\beta_{0}\right)$ will be an $\operatorname{Ar}(1)$. In addition, it is easy to see that

$$
w_{t}(\beta)=\frac{1}{1-\beta L} f_{t}(\beta)
$$

so that

$$
f_{t}(\beta)=w_{t}(\beta)-\beta w_{t-1}(\beta) .
$$

Therefore, we can re-write the score of the MA(1) model as

$$
s_{t}(\beta)=-\left[w_{t}(\beta)-\beta w_{t-1}(\beta)\right] w_{t-1}(\beta),
$$

which coincides with the (minus) score of an $\operatorname{AR}(1)$ model for $w(\beta)$. 
This regression is infeasible, but we can compute $\bar{\delta}_{T}$ as the OLS estimator in the regression of $w_{t}\left(\bar{\beta}_{T}\right)$ on $w_{t-1}\left(\bar{\beta}_{T}\right)$, where $\bar{\beta}_{T}$ is the II estimator of $\beta$ based on the misspecified AR(1) auxiliary model for $x_{t}$.

Unfortunately, $\bar{\delta}_{T}$ is even less efficient than $\bar{\beta}_{T}$. Nevertheless, we can optimally combine those two different consistent but inefficient II estimators. Specifically, we can easily prove that $\tilde{\beta}_{T}=2 \bar{\beta}_{T}-\bar{\delta}_{T}$ is the outcome of a Gauss-Newton iteration, and therefore asymptotically equivalent to the ML estimator. In fact, it is possible to iterate the above procedure and obtain a new estimator $\bar{\delta}_{T}^{1}$ by regressing $w_{t}\left(\tilde{\beta}_{T}\right)$ on $w_{t-1}\left(\tilde{\beta}_{T}\right)$, which preserves asymptotic efficiency. The fixed point of these iterations is the ML estimator.

It turns out that Hannan (1969) proposed a simple iterative frequency domain procedure, which is effectively identical to the iterated indirect inference procedure we have just discussed.

\subsection{Mixed models}

Let us now consider the extension of our iterated II procedure to the ARMA $(1,1)$ model

$$
x_{t}=\alpha x_{t-1}+f_{t}-\beta f_{t-1}, \quad|\alpha|,|\beta|<1, \quad f_{t} \mid x_{t-1}, x_{t-2}, \ldots \sim N(0,1)
$$

The simplest consistent estimator of $\alpha$ and $\beta$ is an indirect inference one based on the misspecified AR(2) auxiliary model

$$
x_{t}=\delta_{1} x_{t-1}+\delta_{2} x_{t-2}+w_{t}, \quad w_{t} \mid x_{t-1}, x_{t-2}, \ldots \sim N(0,1)
$$

(see again Chumacero (2001)). This estimator is asymptotically equivalent to the GMM estimator of $\alpha$ and $\beta$ based on the moment conditions

$$
\begin{aligned}
E\left[\mathbf{m}_{t}(\alpha, \beta) \mid \alpha, \beta\right] & =0 \\
m_{1 t}(\alpha, \beta) & =\left(x_{t}-\frac{(\alpha-\beta)(1-\alpha \beta)}{1-\alpha^{2}} x_{t-1}\right) x_{t-1}, \\
m_{2 t}(\alpha, \beta) & =\left(x_{t}-\frac{\alpha(\alpha-\beta)(1-\alpha \beta)}{1-\alpha^{2}} x_{t-2}\right) x_{t-2} .
\end{aligned}
$$

The exactly identified nature of these moment conditions implies that the indirect inference estimator of $\alpha$ will coincide with the ratio of the second to the first autocorrelation of $x_{t}$, which is always between -1 and 1 . As for the indirect inference estimator of $\beta$, we can obtain it from the first moment condition if we keep $\alpha$ fixed at its indirect inference value. In large samples, this procedure is effectively identical to the indirect inference estimator of $\beta$ described in the previous section obtained by fitting an $\mathrm{AR}(1)$ model to the filtered series $\eta_{t}(\alpha)=x_{t}-\alpha x_{t-1}$.

Once again, we could increase the efficiency with which we estimate $\alpha$ and $\beta$ if we considered higher order $\mathrm{AR}(\mathrm{k})$ models. Unfortunately, for any finite order $k$ those II estimators are generally inefficient relative to the ML estimator, which is effectively based on the moment conditions 


$$
\begin{aligned}
E\left[\mathbf{s}_{t}(\beta) \mid \beta\right] & =0 \\
s_{\alpha t}(\alpha, \beta) & =\left[x_{t}-\nu_{t}(\beta)\right] \frac{\partial \nu_{t}(\alpha, \beta)}{\partial \alpha} \\
s_{\beta t}(\alpha, \beta) & =\left[x_{t}-\nu_{t}(\beta)\right] \frac{\partial \nu_{t}(\alpha, \beta)}{\partial \beta}
\end{aligned}
$$

where

$$
\nu_{t}(\alpha, \beta)=E\left(x_{t} \mid x_{t-1}, x_{t-2}, \ldots ; \alpha, \beta\right)=(\alpha-\beta) \sum_{j=1}^{\infty} \beta^{j-1} x_{t-j}=\frac{(\alpha-\beta) L}{1-\beta L} x_{t}
$$

This highly non-linear estimator can also be related to a couple of auxiliary linear autoregressive models. Specifically, define

$$
f_{t}(\alpha, \beta)=x_{t}-\nu_{t}(\alpha, \beta)=\frac{1-\alpha L}{1-\beta L} x_{t}
$$

as the "innovations" in $x_{t}$. Similarly, let us use the shorthand notation

$$
\begin{aligned}
r_{t}(\beta) & =\frac{\partial \nu_{t+1}(\alpha, \beta)}{\partial \alpha}=\frac{1}{(1-\beta L)} x_{t} \\
w_{t}(\alpha, \beta) & =-\frac{\partial \nu_{t+1}(\alpha, \beta)}{\partial \beta}=\frac{(1-\alpha L)}{(1-\beta L)^{2}} x_{t} .
\end{aligned}
$$

Then it is easy to see that

$$
\begin{aligned}
& s_{\alpha t}(\alpha, \beta)=\left[r_{t}(\beta)-\alpha r_{t-1}(\beta)\right] r_{t-1}(\beta) \\
& s_{\beta t}(\alpha, \beta)=-\left[w_{t}(\alpha, \beta)-\beta w_{t-1}(\alpha, \beta)\right] w_{t-1}(\alpha, \beta)
\end{aligned}
$$

so that we can estimate $\alpha$ for given $\beta$ from the autoregression of $r_{t}(\beta)$ and $\beta$ for given $\alpha$ from the autoregression of $w_{t}(\alpha, \beta)$. Again, these alternative indirect inference estimators will be inefficient when the unknown ARMA parameters are replaced by the indirect inference estimators $\bar{\alpha}_{T}$ and $\bar{\beta}_{T}$ based on the misspecified $\operatorname{Ar}(2)$ auxiliary model for $x_{t}$, but we can combine them by means of a Gauss-Newton iteration of the form

$$
\begin{aligned}
\left(\begin{array}{c}
\tilde{\alpha}_{T} \\
\tilde{\beta}_{T}
\end{array}\right)= & \left(\begin{array}{c}
\bar{\alpha}_{T} \\
\bar{\beta}_{T}
\end{array}\right)+\left\{\frac{1}{T} \sum_{t=1}^{T}\left[\begin{array}{cc}
r_{t-1}^{2}\left(\bar{\beta}_{T}\right) & -r_{t-1}\left(\bar{\beta}_{T}\right) w_{t-1}\left(\bar{\alpha}_{T}, \bar{\beta}_{T}\right) \\
-r_{t-1}\left(\bar{\beta}_{T}\right) w_{t-1}\left(\bar{\alpha}_{T}, \bar{\beta}_{T}\right) & w_{t-1}^{2}\left(\bar{\alpha}_{T}, \bar{\beta}_{T}\right)
\end{array}\right]\right\}^{-1} \\
& \times \frac{1}{T} \sum_{t=1}^{T}\left[\begin{array}{c}
r_{t-1}\left(\bar{\beta}_{T}\right) f_{t}\left(\bar{\alpha}_{T}, \bar{\beta}_{T}\right) \\
-w_{t-1}\left(\bar{\alpha}_{T}, \bar{\beta}_{T}\right) f_{t}\left(\bar{\alpha}_{T}, \bar{\beta}_{T}\right)
\end{array}\right] .
\end{aligned}
$$

Once again, it is possible to iterate the above procedure while preserving asymptotic efficiency, the fixed point of these iterations being the ML estimator.

Analogous procedures apply to general $\operatorname{Arma}(\mathrm{p}, \mathrm{q}) \operatorname{models}^{8}$ if we define

$$
f_{t}(\boldsymbol{\alpha}, \boldsymbol{\beta})=\frac{\alpha_{x}(L)}{\beta_{x}(L)} x_{t}, \quad r_{t}(\boldsymbol{\beta})=\frac{1}{\beta_{x}(L)} x_{t}, \quad w_{t}(\boldsymbol{\alpha}, \boldsymbol{\beta})=\frac{\alpha_{x}(L)}{\beta_{x}^{2}(L)} x_{t} .
$$

\footnotetext{
${ }^{8}$ The stationarity and strict invertibility of the estimated AR and MA polynomials in high order models could be achieved by reparametrising them in terms of partial autocorrelations, as in Barndorff-Nielsen and Schou (1973).
} 
Importantly, the variances, autocovariances and cross-covariances of the different filtered series can be computed much faster in the frequency domain than in the time domain, which makes these iterated indirect inference estimators an ideal match to our spectral estimation techniques (see again Hannan (1969)).

\subsection{Computational gains of the iterated indirect inference procedure}

To assess the performance of our iterated II procedure, we have generated 2,000 samples of 300 observations each (plus 50 initial ones) of univariate Gaussian ARMA $(1,1)$ models with autoregressive parameter $\alpha=.95$ and three possible values for the moving average parameter $\beta$ : $-.5, .5$ and .7 . The first design roughly corresponds to the parameter estimates for the common factor in the empirical application in section 5, while the second one is typical of many observed time series. The last design, in turn, captures those situations in which the autocorrelations are small, but they decay rather slowly to 0. For scaling purposes but without loss of generality, we choose the true value of $\sigma_{f}^{2}$ to be 1 , and estimate this parameter in closed- form from the spectral version of the zero order autocovariance of $f_{t}(\alpha, \beta)$ evaluated at the II estimates.

We use a frequency domain version of the GMM procedure described in the previous section to generate starting values. As a benchmark, we consider the direct maximisation of the spectral log-likelihood function with respect to $\alpha, \beta$ and $\sigma_{f}^{2}$ using the Quasi Newton (QN) procedure in MATLAB's fmincon routine with analytical first derivatives and the spectral estimator of the information matrix in lieu of the Hessian. The convergence criterion for our iterated II procedure is that the $L_{1}$ norm of the parameter changes be less than $10^{-5}$, with a maximum of 100 iterations. Finally, we also look at several procedures that only carry out a fixed number of II iterations. ${ }^{9}$

The three panels of Table 1 present medians and interquartile ranges (IQR) across replications for those different estimators. As can be seen, the efficiency gains increase at a decreasing rate as the number of II iterations increases, despite the fact that all estimators other than the GMM procedure are asymptotically equivalent up to first order. In fact, only a handful of iterations seem to be necessary to achieve full efficiency, except in the case in which the MA root is close to the AR one.

The ranking is completely reversed in terms of computational speed. In particular, our proposed iterated II procedure is an order of magnitude faster than the QN algorithm. Although this is unlikely to make much of a difference for observed series, it becomes crucial when $O(N+1)$ such maximisations are required at each stage of the EM algorithm we describe next.

\footnotetext{
${ }^{9}$ We also considered mixed procedures that switch to QN after a fixed number of II iterations, but given that they typically converge to the same estimates as the direct ML procedure after giving up most of the computational gains, we do not discuss them separately.
} 


\section{Spectral EM algorithm}

As we mentioned in the introduction, the EM algorithm in Dempster, Laird and Rubin (1977) adapted to static factor models by Rubin and Thayer (1982) was successfully employed to handle a very large dataset of stock returns by Lehmann and Modest (1988). Shumway and Stoffer (1982), Watson and Engle (1983) and Quah and Sargent (1993) also applied the algorithm in the time domain to dynamic factor models and some generalisations, but they restricted common and specific factors to follow low order AR processes.

We saw before that the spectral density matrix of a dynamic single factor model has the structure of the unconditional covariance matrix of a static factor model, but with different common and idiosyncratic variances for each frequency. Demos and Sentana (1998) applied a time domain version of the EM algorithm to conditionally heteroskedastic factor models in which the common factors followed GARCH-type processes. We could easily adapt their algorithm to models with white noise idiosyncratic factors and contemporaneous effects of the common factors on the observed variables if we replaced the subscript $t$ for time with the subscript $j$ for frequency. However, since we want to consider more complex models, we need to do some additional algebra.

\subsection{Complete log-likelihood function}

Consider a situation in which the common factor $x_{t}$ was also observed. The joint spectral density of $\mathbf{y}_{t}$ and $x_{t}$, which is given by

$$
\left[\begin{array}{ll}
\mathbf{G}_{\mathbf{y y}}(\lambda) & \mathbf{G}_{\mathbf{y} x}(\lambda) \\
\mathbf{G}_{\mathbf{y} x}^{*}(\lambda) & G_{x x}(\lambda)
\end{array}\right]=\left[\begin{array}{cc}
\mathbf{c}\left(e^{-i \lambda}\right) G_{x x}(\lambda) \mathbf{c}^{\prime}\left(e^{i \lambda}\right)+\mathbf{G}_{\mathbf{u u}}(\lambda) & \mathbf{c}\left(e^{-i \lambda}\right) G_{x x}(\lambda) \\
G_{x x}(\lambda) \mathbf{c}^{\prime}\left(e^{i \lambda}\right) & G_{x x}(\lambda)
\end{array}\right],
$$

could be diagonalised as

$$
\left[\begin{array}{cc}
\mathbf{I}_{N} & \mathbf{c}\left(e^{-i \lambda}\right) \\
\mathbf{0} & 1
\end{array}\right]\left[\begin{array}{cc}
\mathbf{G}_{\mathbf{u u}}(\lambda) & \mathbf{0} \\
\mathbf{0} & G_{x x}(\lambda)
\end{array}\right]\left[\begin{array}{cc}
\mathbf{I}_{N} & \mathbf{0} \\
\mathbf{c}^{\prime}\left(e^{i \lambda}\right) & 1
\end{array}\right]
$$

with

$$
\left|\left[\begin{array}{cc}
\mathbf{I}_{N} & \mathbf{0} \\
\mathbf{c}^{\prime}\left(e^{i \lambda}\right) & 1
\end{array}\right]\right|=1
$$

and

$$
\left[\begin{array}{cc}
\mathbf{I}_{N} & \mathbf{0} \\
\mathbf{c}^{\prime}\left(e^{i \lambda}\right) & 1
\end{array}\right]^{-1}=\left[\begin{array}{cc}
\mathbf{I}_{N} & \mathbf{0} \\
-\mathbf{c}^{\prime}\left(e^{i \lambda}\right) & 1
\end{array}\right]
$$

Let us define as $\left[\mathbf{Z}^{\mathbf{y}} \mid \mathbf{z}^{x}\right]$ as the Fourier transform of the $T \times(N+1)$ matrix $\left[\mathbf{y}_{1}, \ldots, \mathbf{y}_{N}, \mathbf{x}\right]=$ $[\mathbf{Y} \mid \mathbf{x}]$ so that the joint periodogram of $\mathbf{y}_{t}$ and $x_{t}$ at frequency $\lambda_{j}$ could be quickly computed as

$$
2 \pi\left(\begin{array}{c}
\mathbf{z}_{j}^{\mathbf{y}} \\
z_{j}^{x}
\end{array}\right)\left(\begin{array}{ll}
\mathbf{z}_{j}^{\mathbf{y} *} & z_{j}^{x *}
\end{array}\right),
$$


where we have implicitly assumed that either the elements of $\mathbf{y}$ have zero mean, or else that they have been previously demeaned by subtracting their sample averages.

In this notation, the spectral approximation to the joint log-likelihood function would become

$$
\begin{aligned}
& l(\mathbf{y}, x)=-\frac{(N+1) T}{2} \ln (2 \pi)-\frac{1}{2} \sum_{j=0}^{T-1} \ln \left|\left[\begin{array}{cc}
\mathbf{G}_{\mathbf{y y}}(\lambda) & \mathbf{G}_{\mathbf{y} x}\left(\lambda_{j}\right) \\
\mathbf{G}_{\mathbf{y} x}^{*}\left(\lambda_{j}\right) & G_{x x}\left(\lambda_{j}\right)
\end{array}\right]\right| \\
& -\frac{2 \pi}{2} \sum_{j=0}^{T-1}\left(\begin{array}{ll}
\mathbf{z}_{j}^{\mathbf{y} *} & z_{j}^{x *}
\end{array}\right)\left[\begin{array}{cc}
\mathbf{I}_{N} & \mathbf{0} \\
-\mathbf{c}^{\prime}\left(e^{i \lambda_{j}}\right) & 1
\end{array}\right]\left[\begin{array}{cc}
\mathbf{G}_{\mathbf{u u}}^{-1}\left(\lambda_{j}\right) & \mathbf{0} \\
\mathbf{0} & G_{x x}^{-1}\left(\lambda_{j}\right)
\end{array}\right]\left[\begin{array}{cc}
\mathbf{I}_{N} & \mathbf{c}\left(e^{-i \lambda_{j}}\right) \\
\mathbf{0} & 1
\end{array}\right]\left(\begin{array}{c}
\mathbf{z}_{j}^{\mathbf{y}} \\
z_{j}^{x}
\end{array}\right) \\
& =-\frac{N T}{2} \ln (2 \pi)-\frac{1}{2} \sum_{j=0}^{T-1} \ln \left|\mathbf{G}_{\mathbf{u u}}\left(\lambda_{j}\right)\right|-\frac{2 \pi}{2} \sum_{j=0}^{T-1} \mathbf{z}_{j}^{\mathbf{u} *} \mathbf{G}_{\mathbf{u u}}^{-1}\left(\lambda_{j}\right) \mathbf{z}_{j}^{\mathbf{u}} \\
& -\frac{T}{2} \ln (2 \pi)-\frac{1}{2} \sum_{j=0}^{T-1} \ln \left|G_{x x}\left(\lambda_{j}\right)\right|-\frac{2 \pi}{2} \sum_{j=0}^{T-1} G_{x x}^{-1}\left(\lambda_{j}\right) z_{j}^{x} z_{j}^{x *} \\
& =\sum_{i=1}^{N}\left[-\frac{T}{2} \ln (2 \pi)-\frac{1}{2} \sum_{j=0}^{T-1} \ln \left|G_{u_{i} u_{i}}\left(\lambda_{j}\right)\right|-\frac{2 \pi}{2} \sum_{j=0}^{T-1} G_{u_{i} u_{i}}^{-1}\left(\lambda_{j}\right) z_{j}^{u_{i}} z_{j}^{u_{i} *}\right] \\
& -\frac{T}{2} \ln (2 \pi)-\frac{1}{2} \sum_{j=0}^{T-1} \ln \left|G_{x x}\left(\lambda_{j}\right)\right|-\frac{2 \pi}{2} \sum_{j=0}^{T-1} G_{x x}^{-1}\left(\lambda_{j}\right) z_{j}^{x} z_{j}^{x *} \\
& =\sum_{i=1}^{N} l\left(\mathbf{y}_{i} \mid \mathbf{x}\right)+l(\mathbf{x})=l(\mathbf{Y} \mid \mathbf{x})+l(\mathbf{x}),
\end{aligned}
$$

where $^{10}$

$$
z_{j}^{u_{i}}=z_{j}^{y_{i}}-c_{i}\left(e^{-i \lambda_{j}}\right) z_{j}^{x}=z_{j}^{y_{i}}-\sum_{k=-m}^{n} c_{i, k} e^{-i k \lambda} z_{j}^{x},
$$

so that

$$
\begin{aligned}
z_{j}^{u_{i}} z_{j}^{u_{i} *} & =z_{j}^{y_{i}} z_{j}^{y_{i} *}-c_{i}\left(e^{-i \lambda_{j}}\right) z_{j}^{x} z_{j}^{y_{i} *}-c_{i}\left(e^{i \lambda_{j}}\right) z_{j}^{y_{i}} z_{j}^{x *}+c_{i}\left(e^{-i \lambda_{j}}\right) c_{i}\left(e^{i \lambda_{j}}\right) z_{j}^{x} z_{j}^{x *} \\
& =I_{y_{i} y_{i}}\left(\lambda_{j}\right)-c_{i}\left(e^{-i \lambda_{j}}\right) I_{x y_{i}}\left(\lambda_{j}\right)-c_{i}\left(e^{i \lambda_{j}}\right) I_{y_{i} x}\left(\lambda_{j}\right)+c_{i}\left(e^{-i \lambda_{j}}\right) c_{i}\left(e^{i \lambda_{j}}\right) I_{x x}\left(\lambda_{j}\right)=I_{u_{i} u_{i}}\left(\lambda_{j}\right) .
\end{aligned}
$$

In this way, we have decomposed the joint log-likelihood function of $\mathbf{y}_{1}, \ldots, \mathbf{y}_{N}$ and $\mathbf{x}$ as the sum of the marginal log-likelihood of $\mathbf{x}$ in (9) and the log-likelihood function of $\mathbf{y}_{1}, \ldots, \mathbf{y}_{N}$ given $\mathbf{x}, l(\mathbf{Y} \mid \mathbf{x})$, which in turn can be decomposed as the sum of $N$ univariate components in (8) by exploiting the diagonality of $\mathbf{G}_{\mathbf{u u}}\left(\lambda_{j}\right)$.

Importantly, these expressions can be computed using real arithmetic only since

$$
c_{i}\left(e^{-i \lambda_{j}}\right) I_{x y_{i}}\left(\lambda_{j}\right)+c_{i}\left(e^{i \lambda_{j}}\right) I_{y_{i} x}\left(\lambda_{j}\right)=2 \Re\left[c_{i}\left(e^{-i \lambda_{j}}\right) I_{x y_{i}}\left(\lambda_{j}\right)\right]
$$

and

$$
c_{i}\left(e^{-i \lambda_{j}}\right) c_{i}\left(e^{i \lambda_{j}}\right) I_{x x}\left(\lambda_{j}\right)=\left\|c_{i}\left(e^{-i \lambda_{j}}\right)\right\|^{2} I_{x x}\left(\lambda_{j}\right)
$$

\footnotetext{
${ }^{10}$ We could have expressed those log-likelihood in terms of $I_{x x}\left(\lambda_{j}\right)=z_{j}^{x} z_{j}^{x *}, \mathbf{I}_{\mathbf{u u}}(\lambda)=\mathbf{z}_{j}^{\mathbf{u}} \mathbf{z}_{j}^{\mathbf{u} *}$ and $\mathbf{I}_{\mathbf{u} x}(\lambda)=\mathbf{z}_{j}^{\mathbf{u}} z_{j}^{x *}$, but for the EM algorithm it is more convenient to work with the underlying complex random variables.
} 
Let us classify the parameters into three blocks:

1. the parameters that characterise the spectral density of $x_{t}: \boldsymbol{\theta}_{x}$,

2. the parameters that characterise the spectral density of $\mathbf{u}_{t}: \boldsymbol{\psi}=\left(\psi_{1}, \ldots, \psi_{N}\right)^{\prime}$ and $\boldsymbol{\theta}_{\mathbf{u}}=\left(\boldsymbol{\theta}_{u_{i}}^{\prime}, \ldots, \boldsymbol{\theta}_{u_{N}}^{\prime}\right)^{\prime}$

3. the parameters that characterise the dynamic idiosyncratic impact of the common factors on each observed variable: $\mathbf{c}=\left(\mathbf{c}_{1 .}^{\prime}, \ldots, \mathbf{c}_{i .}^{\prime}, \ldots, \mathbf{c}_{N}^{\prime} .\right)^{\prime}$, where $\mathbf{c}_{i .}^{\prime}=\left(c_{i,-m}, \ldots, c_{i, 0}, \ldots, c_{i, n}\right)$.

Importantly, $\boldsymbol{\theta}_{x}$ only appear in (9) while $\boldsymbol{\theta}_{\mathbf{u}}$ and $\mathbf{c}$ appear in (8). This sequential cut on the joint spectral density confirms that $z^{x}$ and therefore $x_{t}$ would be weakly exogenous for $\boldsymbol{\psi}, \boldsymbol{\theta}_{\mathbf{u}}$ and c (see Engle, Hendry and Richard (1983)). Moreover, the fact that $f_{t}$ is uncorrelated at all leads and lags with $\mathbf{v}_{t}$ implies that $x_{t}$ would be strongly exogenous too.

We can also exploit the aforementioned log-likelihood decomposition to obtain the score of the complete log-likelihood function. In this way, we can write

$$
\begin{gathered}
\frac{\partial l(\mathbf{Y}, \mathbf{x})}{\partial \boldsymbol{\theta}_{x}}=\frac{\partial l(\mathbf{x})}{\partial \boldsymbol{\theta}_{x}}=\frac{1}{2} \sum_{j=0}^{T-1} \frac{\partial G_{x x}\left(\lambda_{j}\right)}{\partial \boldsymbol{\theta}_{x}} G_{x x}^{-2}\left(\lambda_{j}\right)\left[2 \pi z_{j}^{x} z_{j}^{x *}-G_{x x}\left(\lambda_{j}\right)\right] \\
\frac{\partial l(\mathbf{Y}, \mathbf{x})}{\partial \boldsymbol{\theta}_{u_{i}}}=\frac{\partial l\left(\mathbf{y}_{i} \mid \mathbf{x}\right)}{\partial \boldsymbol{\theta}_{u_{i}}}=\frac{1}{2} \sum_{j=0}^{T-1} \frac{\partial G_{u_{i} u_{i}}\left(\lambda_{j}\right)}{\partial \boldsymbol{\theta}_{u_{i}}} G_{u_{i} u_{i}}^{-2}\left(\lambda_{j}\right)\left[2 \pi z_{j}^{u_{i}} z_{j}^{u_{i} *}-G_{u_{i} u_{i}}\left(\lambda_{j}\right)\right] \\
\frac{\partial l(\mathbf{Y}, \mathbf{x})}{\partial c_{i, k}}=\frac{\partial l\left(\mathbf{y}_{i} \mid \mathbf{x}\right)}{\partial c_{i, k}}=\frac{2 \pi}{2} \sum_{j=0}^{T-1} G_{u_{i} u_{i}}^{-1}\left(\lambda_{j}\right)\left[z_{j}^{u_{i}} e^{i k \lambda_{j}} z_{j}^{x *}+e^{-i k \lambda_{j}} z_{j}^{x} z_{j}^{u_{i} *}\right] \\
=\frac{2 \pi}{2} \sum_{j=0}^{T-1} G_{u_{i} u_{i}}^{-1}\left(\lambda_{j}\right)\left[\left(z_{j}^{y_{i}}-\sum_{l=-m}^{n} c_{i, l} e^{-i l \lambda} z_{j}^{x}\right) e^{i k \lambda_{j}} z_{j}^{x *}+e^{-i k \lambda_{j}} z_{j}^{x}\left(z_{j}^{y_{i}^{*}}-\sum_{l=-m}^{n} c_{i, l} e^{i l \lambda} z_{j}^{x *}\right)\right]
\end{gathered}
$$

where we have used the fact that

$$
\frac{\partial z_{j}^{u_{i}}}{\partial c_{i, k}}=-e^{-i k \lambda} z_{j}^{x}
$$

in view of (10).

Expression (11a) confirms that the MLE of $\boldsymbol{\theta}_{x}$ would be obtained from the marginal loglikelihood of $x_{t}$. In models with pure AR processes, such as the one discussed in appendix $\mathrm{F}$ or in Fiorentini, Galesi and Sentana (2016), one could easily obtain closed form expressions for the estimators. However, there are no closed form solutions for models with MA components because $G_{x x}\left(\lambda_{j}\right)$ also depends on $\boldsymbol{\theta}_{x}$, so in principle we would have to resort to the numerical optimisation of (9). In this context, the swift iterated indirect inference procedures in section 3 would prove very useful because they are asymptotically equivalent to ML.

Analogous comments apply to the dynamic parameters that appear in $\boldsymbol{\theta}_{u_{i}}$ for a given value of $\mathbf{c}_{i}$. in view of (11b). 
Finally, (11c) would allow us to obtain the ML estimators of $\mathbf{c}_{i}$. for given values of $\boldsymbol{\theta}_{u_{i}}$. In particular, if we write together the derivatives for $c_{i, k}$ for $k=-m, \ldots, 0, \ldots, n$ we end up with the "weighted" normal equations:

$$
\begin{aligned}
& \sum_{j=0}^{T-1}\left[G _ { u _ { i } u _ { i } } ^ { - 1 } ( \lambda _ { j } ) \left(\begin{array}{cc}
e^{i m \lambda_{j}} z_{j}^{x} z_{j}^{x *} e^{-i m \lambda_{j}}+e^{i m \lambda_{j}} z_{j}^{x} z_{j}^{x *} e^{-i m \lambda_{j}} & \ldots \\
\vdots & \ddots \\
e^{i m \lambda_{j}} z_{j}^{x} z_{j}^{x *} e^{i n \lambda_{j}}+e^{-i n \lambda_{j}} z_{j}^{x} z_{j}^{x *} e^{-i m \lambda_{j}} & \ldots
\end{array}\right.\right. \\
& \left.\left.\begin{array}{c}
e^{-i n \lambda_{j}} z_{j}^{x} z_{j}^{x *} e^{-i m \lambda_{j}}+e^{i m \lambda_{j}} z_{j}^{x} z_{j}^{x *} e^{i n \lambda_{j}} \\
\vdots \\
e^{-i n \lambda_{j}} z_{j}^{x} z_{j}^{x *} e^{i n \lambda_{j}}+e^{-i n \lambda_{j}} z_{j}^{x} z_{j}^{x *} e^{i n \lambda_{j}}
\end{array}\right)\right]\left(\begin{array}{c}
\tilde{c}_{i,-m} \\
\vdots \\
\tilde{c}_{i, n}
\end{array}\right) \\
& =\sum_{j=0}^{T-1} G_{u_{i} u_{i}}^{-1}\left(\lambda_{j}\right)\left(\begin{array}{c}
z_{j}^{y_{i}} z_{j}^{x *} e^{-i m \lambda_{j}}+z_{j}^{y_{i} *} z_{j}^{x} e^{i m \lambda_{j}} \\
\vdots \\
z_{j}^{y_{i}} z_{j}^{x *} e^{i n \lambda_{j}}+z_{j}^{y_{i} *} z_{j}^{x} e^{-i n \lambda_{j}}
\end{array}\right) .
\end{aligned}
$$

Thus, unrestricted MLE's of $\mathbf{c}$ for given values of $\boldsymbol{\theta}_{u_{i}}$ could be obtained from $N$ univariate distributed lag weighted least squares regressions of each $y_{i t}$ on $x_{t}$ that take into account the residual serial correlation in $u_{i t}$. Interestingly, given that $G_{u_{i} u_{i}}\left(\lambda_{j}\right)$ is real, the above system of equations would not involve complex arithmetic. In addition, the terms in $\psi_{i}$ would cancel, so the WLS procedure would only depend on the dynamic elements in $\boldsymbol{\theta}_{u_{i}}$.

We could then carry out a zig-zag procedure that would estimate $\mathbf{c}_{i}$. and $\psi_{i}$ for given $\boldsymbol{\theta}_{u_{i}}$, and then $\boldsymbol{\theta}_{u_{i}}$ for a given $\mathbf{c}_{i}$. and $\psi_{i}$. This would represent the spectral analogue to the CochraneOrcutt (1949) procedure. Obviously, iterations would be unnecessary when $\mathbf{G}_{\mathbf{u u}}\left(\lambda_{j}\right)$ is in fact constant, so that the idiosyncratic terms are static, in which case the equations could be written in terms of the elements of the covariance and first autocovariance matrices of $\mathbf{y}_{t}$ and $x_{t}$.

Unfortunately, we would have to resort once again to numerical optimisation in models with MA components, which would be far more taxing than in the case of the common factor because there would be $N$ such optimisations at each Cochrane-Orcutt iteration. Once again, the iterated indirect inference procedures in section 3 would also prove very useful in this context.

\subsection{Expected log-likelihood function}

In practice, of course, we do not observe $x_{t}$. Nevertheless, the EM algorithm can be used to obtain values for $\boldsymbol{\theta}$ as close to the optimum as desired. At each iteration, the EM algorithm maximises the expected value of $l\left(\mathbf{y}_{1}, \ldots, \mathbf{y}_{N} \mid \mathbf{x}\right)+l(\mathbf{x})$ conditional on $\mathbf{Y}$ and the current parameter estimates, $\boldsymbol{\theta}^{(n)}$. The rationale stems from the fact that $l\left(\mathbf{y}_{1}, \ldots, \mathbf{y}_{N}, \mathbf{x}\right)$ can also be factorized as $l\left(\mathbf{y}_{1}, \ldots, \mathbf{y}_{N}\right)+l\left(\mathbf{x} \mid \mathbf{y}_{1}, \ldots, \mathbf{y}_{N}\right)$. Since the expected value of the latter, conditional on $\mathbf{Y}$ and $\boldsymbol{\theta}^{(n)}$, reaches a maximum at $\boldsymbol{\theta}=\boldsymbol{\theta}^{(n)}$, any increase in the expected value of $l\left(\mathbf{y}_{1}, \ldots, \mathbf{y}_{N}, \mathbf{x}\right)$ must represent an increase in $l\left(\mathbf{y}_{1}, \ldots, \mathbf{y}_{N}\right)$. This is the generalised EM principle. 
In the $E$ step we must compute

$$
\begin{aligned}
E\left[l(\mathbf{x}) \mid \mathbf{Z}^{\mathbf{y}}, \boldsymbol{\theta}^{(n)}\right] & =-\frac{T}{2} \ln (2 \pi)-\frac{1}{2} \sum_{j=0}^{T-1} \ln \left|G_{x x}\left(\lambda_{j}\right)\right|-\frac{2 \pi}{2} \sum_{j=0}^{T-1} G_{x x}^{-1}\left(\lambda_{j}\right) E\left[z_{j}^{x} z_{j}^{x *} \mid \mathbf{Z}^{\mathbf{y}}, \boldsymbol{\theta}^{(n)}\right], \\
E\left[l\left(\mathbf{y}_{i} \mid \mathbf{x}\right) \mid \mathbf{Z}^{\mathbf{y}}, \boldsymbol{\theta}^{(n)}\right] & =-\frac{T}{2} \ln (2 \pi)-\frac{1}{2} \sum_{j=0}^{T-1} \ln \left|G_{u_{i} u_{i}}\left(\lambda_{j}\right)\right|-\frac{2 \pi}{2} \sum_{j=0}^{T-1} G_{u_{i} u_{i}}^{-1}\left(\lambda_{j}\right) E\left[z_{j}^{u_{i}} z_{j}^{u_{i} *} \mid \mathbf{Z}^{\mathbf{y}}, \boldsymbol{\theta}^{(n)}\right] .
\end{aligned}
$$

But

$$
\begin{aligned}
E\left[z_{j}^{x} z_{j}^{x *} \mid \mathbf{Z}_{y}, \boldsymbol{\theta}^{(n)}\right] & =z_{j}^{x_{K}}\left(\boldsymbol{\theta}^{(n)}\right) z_{j}^{x_{K} *}\left(\boldsymbol{\theta}^{(n)}\right)+E\left\{\left[z_{j}^{x}-z_{j}^{x_{K}}\left(\boldsymbol{\theta}^{(n)}\right)\right]\left[z_{j}^{x *}-z_{j}^{x *}\left(\boldsymbol{\theta}^{(n)}\right)\right] \mid \mathbf{z}_{j}^{\mathbf{y}}, \boldsymbol{\theta}^{(n)}\right\} \\
& =I_{x^{K} x^{K}}^{(n)}\left(\lambda_{j}\right)+\omega^{(n)}\left(\lambda_{j}\right),
\end{aligned}
$$

where

$$
\begin{aligned}
z_{j}^{x_{K}}(\boldsymbol{\theta})=E\left[z_{j}^{x} \mid \mathbf{Z}^{\mathbf{y}}, \boldsymbol{\theta}\right] & =G_{x x}\left(\lambda_{j}\right) \mathbf{c}^{\prime}\left(e^{i \lambda_{j}}\right) \mathbf{G}_{\mathbf{y y}}^{-1}\left(\lambda_{j}\right) \mathbf{z}_{j}^{\mathbf{y}}, \\
E\left\{\left[z_{j}^{x}-z_{j}^{x_{K}}(\boldsymbol{\theta})\right]\left[z_{j}^{x_{K} *}-z_{j}^{x *}(\boldsymbol{\theta})\right] \mid \mathbf{Z}^{\mathbf{y}}, \boldsymbol{\theta}\right\} & =\omega\left(\lambda_{j}\right),
\end{aligned}
$$

and

$$
\begin{aligned}
I_{x^{K} x^{K}}\left(\lambda_{j}\right) & =2 \pi G_{x x}^{2}\left(\lambda_{j}\right) \mathbf{c}^{\prime}\left(e^{i \lambda_{j}}\right) \mathbf{G}_{\mathbf{y y}}^{-1}\left(\lambda_{j}\right) \mathbf{I}_{\mathbf{y y}}\left(\lambda_{j}\right) \mathbf{G}_{\mathbf{y y}}^{-1}\left(\lambda_{j}\right) \mathbf{c}\left(e^{-i \lambda_{j}}\right) \\
& =2 \pi \omega^{2}\left(\lambda_{j}\right) \mathbf{c}^{\prime}\left(e^{i \lambda_{j}}\right) \mathbf{G}_{\mathbf{u u}}^{-1}\left(\lambda_{j}\right) \mathbf{I}_{\mathbf{y y}}\left(\lambda_{j}\right) \mathbf{G}_{\mathbf{u u}}^{-1}\left(\lambda_{j}\right) \mathbf{c}\left(e^{-i \lambda_{j}}\right) .
\end{aligned}
$$

is the periodogram of the smoothed values of the common factor.

In turn, if we define

$$
\mathbf{I}_{\mathbf{y} x^{K}}\left(\lambda_{j}\right)=\mathbf{I}_{\mathbf{y y}}\left(\lambda_{j}\right) \mathbf{G}_{\mathbf{y y}}^{-1}\left(\lambda_{j}\right) \mathbf{c}\left(e^{-i \lambda_{j}}\right) G_{x x}\left(\lambda_{j}\right)=\mathbf{I}_{\mathbf{y y}}\left(\lambda_{j}\right) \mathbf{G}_{\mathbf{u u}}^{-1}\left(\lambda_{j}\right) \mathbf{c}\left(e^{-i \lambda_{j}}\right) \omega\left(\lambda_{j}\right)
$$

as the cross-periodogram between the observed series $\mathbf{y}$ and the smoothed values of the common factor, we will have that

$$
\begin{gathered}
\mathbf{I}_{\mathbf{u u}}^{(n)}\left(\lambda_{j}\right)=E\left[\mathbf{z}_{j}^{\mathbf{u}} \mathbf{z}_{j}^{\mathbf{u} *} \mid \mathbf{Z}^{\mathbf{y}}, \boldsymbol{\theta}^{(n)}\right]=E\left\{\left[\mathbf{z}_{j}^{\mathbf{y}}-\mathbf{c}\left(e^{-i \lambda_{j}}\right) z_{j}^{x}\right]\left[\mathbf{z}_{j}^{\mathbf{y} *}-z_{j}^{x *} \mathbf{c}^{\prime}\left(e^{i \lambda_{j}}\right)\right] \mid \mathbf{Z}^{\mathbf{y}}, \boldsymbol{\theta}^{(n)}\right\} \\
=\left[\mathbf{z}_{j}^{\mathbf{y}}-\mathbf{c}\left(e^{-i \lambda_{j}}\right) z_{j}^{x_{K}}\left(\boldsymbol{\theta}^{(n)}\right)\right]\left[\mathbf{z}_{j}^{\mathbf{y} *}-\mathbf{c}^{\prime}\left(e^{i \lambda_{j}}\right) z_{j}^{x_{K} *}\left(\boldsymbol{\theta}^{(n)}\right)\right]+\mathbf{c}\left(e^{-i \lambda_{j}}\right) \omega^{(n)}\left(\lambda_{j}\right) \mathbf{c}^{\prime}\left(e^{i \lambda_{j}}\right) \\
=\mathbf{I}_{\mathbf{y} \mathbf{y}}\left(\lambda_{j}\right)-\mathbf{I}_{\mathbf{y} x^{K}}^{(n)}\left(\lambda_{j}\right) \mathbf{c}^{\prime}\left(e^{i \lambda_{j}}\right)-\mathbf{c}\left(e^{-i \lambda_{j}}\right) \mathbf{I}_{x^{K} \mathbf{y}}^{(n)}\left(\lambda_{j}\right)+\mathbf{c}\left(e^{-i \lambda_{j}}\right)\left[I_{x^{K} x^{K}}^{(n)}\left(\lambda_{j}\right)+\omega^{(n)}\left(\lambda_{j}\right)\right] \mathbf{c}^{\prime}\left(e^{i \lambda_{j}}\right),
\end{gathered}
$$

which resembles the expected value of $\mathbf{I}_{\mathbf{u u}}\left(\lambda_{j}\right)$ but the values at which the expectations are evaluated are generally different from the values at which the distributed lags are computed.

For the $i^{\text {th }}$ series, this expression reduces to

$$
\begin{gathered}
I_{u_{i} u_{i}}^{(n)}\left(\lambda_{j}\right)=E\left[z_{j}^{u_{i}} z_{j}^{u_{i} *} \mid \mathbf{Z}_{y}, \boldsymbol{\theta}^{(n)}\right]=\left[z_{j}^{y_{i}}-c_{i}\left(e^{-i \lambda_{j}}\right) z_{j}^{x_{K}}\left(\boldsymbol{\theta}^{(n)}\right)\right]\left[z_{j}^{y_{i} *}-c_{i}\left(e^{i \lambda_{j}}\right) z_{j}^{x_{K} *}\left(\boldsymbol{\theta}^{(n)}\right)\right] \\
+\omega^{(n)}\left(\lambda_{j}\right) c_{i}\left(e^{-i \lambda_{j}}\right) c_{i}\left(e^{i \lambda_{j}}\right) \\
=I_{y_{i} y_{i}}\left(\lambda_{j}\right)-c_{i}\left(e^{-i \lambda_{j}}\right) I_{x^{K} y_{i}}^{(n)}\left(\lambda_{j}\right)-I_{y_{i} x^{K}}^{(n)}\left(\lambda_{j}\right) c_{i}\left(e^{i \lambda_{j}}\right)+\left[I_{x^{K} x^{K}}^{(n)}\left(\lambda_{j}\right)+\omega^{(n)}\left(\lambda_{j}\right)\right] c_{i}\left(e^{-i \lambda_{j}}\right) c_{i}\left(e^{i \lambda_{j}}\right) .
\end{gathered}
$$


Therefore, if we put all these expressions together we end up with

$$
\begin{gathered}
E\left[l(\mathbf{x}) \mid \mathbf{Y}, \boldsymbol{\theta}^{(n)}\right]=-\frac{T}{2} \ln (2 \pi)-\frac{1}{2} \sum_{j=0}^{T-1} \ln \left|G_{x x}\left(\lambda_{j}\right)\right|-\frac{2 \pi}{2} \sum_{j=0}^{T-1} G_{x x}^{-1}\left(\lambda_{j}\right)\left[I_{x^{K} x^{K}}^{(n)}\left(\lambda_{j}\right)+\omega^{(n)}\left(\lambda_{j}\right)\right], \\
E\left[l\left(\mathbf{y}_{i} \mid \mathbf{x}\right) \mid \mathbf{Y}, \boldsymbol{\theta}^{(n)}\right]=-\frac{T}{2} \ln (2 \pi)-\frac{1}{2} \sum_{j=0}^{T-1} \ln \left|G_{u_{i} u_{i}}\left(\lambda_{j}\right)\right|-\frac{2 \pi}{2} \sum_{j=0}^{T-1} G_{u_{i} u_{i}}^{-1}\left(\lambda_{j}\right) I_{u_{i} u_{i}}^{(n)}\left(\lambda_{j}\right) .
\end{gathered}
$$

We can then maximise $E\left[l(\mathbf{x}) \mid \mathbf{Y}, \boldsymbol{\theta}^{(n)}\right]$ in (15) with respect to $\boldsymbol{\theta}_{x}$ to update those parameters. Similarly, we can maximise $E\left[l\left(\mathbf{y}_{i} \mid \mathbf{x}\right) \mid \mathbf{Y}, \boldsymbol{\theta}^{(n)}\right]$ in (16) with respect to $\mathbf{c}_{i}$., $\psi_{i}$ and $\boldsymbol{\theta}_{u_{i}}$ to update those parameters.

In order to conduct those maximisations, we need the scores of the expected log-likelihood functions.

Given that (15) is obtained from (9) by simply replacing $z_{j}^{x} z_{j}^{x *}$ with its conditional expected value $I_{x^{K}{ }^{K}{ }^{K}}^{(n)}\left(\lambda_{j}\right)+\omega^{(n)}\left(\lambda_{j}\right)$, it is easy to see that

$$
\frac{\partial E\left[l(\mathbf{x}) \mid \mathbf{Y}, \boldsymbol{\theta}^{(n)}\right]}{\partial \boldsymbol{\theta}_{x}}=\frac{1}{2} \sum_{j=0}^{T-1} \frac{\partial G_{x x}\left(\lambda_{j}\right)}{\partial \boldsymbol{\theta}_{x}} G_{x x}^{-2}\left(\lambda_{j}\right)\left\{2 \pi\left[I_{x^{K} x^{K}}^{(n)}\left(\lambda_{j}\right)+\omega^{(n)}\left(\lambda_{j}\right)\right]-G_{x x}\left(\lambda_{j}\right)\right\},
$$

which, not surprisingly, coincides with the the expected value of (11a) given $\mathbf{Y}$ and the current parameter estimates, $\boldsymbol{\theta}^{(n)}$.

It is also straightforward to modify the indirect inference procedures discussed in section 3 to handle models with ARMA terms if we replace the periodogram of the common factor by its expected value given observables, which coincides with sum of the periodogram of the smoothed values of the factor and its estimation error. Those periodograms can be obtained in no time in the $E$ step of the algorithm from the minimal "sufficient statistics" discussed in appendix B.

Similar expressions would apply to the dynamic parameters that appear in $\boldsymbol{\theta}_{u_{i}}$ and $\psi_{i}$ for a given value of $\mathbf{c}_{i}$.

Finally, the derivatives of (16) with respect to $c_{i, k}$ for $k=-m, \ldots, 0, \ldots, n$ for fixed values of $\boldsymbol{\theta}_{u_{i}}$ will give rise to the modified "weighted" normal equations:

$$
\begin{aligned}
& \sum_{j=0}^{T-1}\left[G _ { u _ { i } u _ { i } } ^ { - 1 } ( \lambda _ { j } ) \left(\begin{array}{cc}
e^{i m \lambda_{j}}\left[I_{x^{K} x^{K}}^{(n)}\left(\lambda_{j}\right)+\omega^{(n)}\left(\lambda_{j}\right)\right] e^{-i m \lambda_{j}}+e^{i m \lambda_{j}}\left[I_{x^{K} x^{K}}^{(n)}\left(\lambda_{j}\right)+\omega^{(n)}\left(\lambda_{j}\right)\right] e^{-i m \lambda_{j}} & \ldots \\
\vdots & \ddots \\
e^{i m \lambda_{j}}\left[I_{x^{K} x^{K}}^{(n)}\left(\lambda_{j}\right)+\omega^{(n)}\left(\lambda_{j}\right)\right] e^{i n \lambda_{j}}+e^{-i n \lambda_{j}}\left[I_{x^{K} x^{K}}^{(n)}\left(\lambda_{j}\right)+\omega^{(n)}\left(\lambda_{j}\right)\right] e^{-i m \lambda_{j}} & \ldots
\end{array}\right.\right. \\
& \left.\left.\begin{array}{c}
e^{-i n \lambda_{j}}\left[I_{x^{K} x^{K}}^{(n)}\left(\lambda_{j}\right)+\omega^{(n)}\left(\lambda_{j}\right)\right] e^{-i m \lambda_{j}}+e^{i m \lambda_{j}}\left[I_{x^{K} x^{K}}^{(n)}\left(\lambda_{j}\right)+\omega^{(n)}\left(\lambda_{j}\right)\right] e^{i n \lambda_{j}} \\
\vdots \\
e^{-i n \lambda_{j}}\left[I_{x^{K} x^{K}}^{(n)}\left(\lambda_{j}\right)+\omega^{(n)}\left(\lambda_{j}\right)\right] e^{i n \lambda_{j}}+e^{-i n \lambda_{j}}\left[I_{x^{K}{ }^{K}{ }^{K}}^{(n)}\left(\lambda_{j}\right)+\omega^{(n)}\left(\lambda_{j}\right)\right] e^{i n \lambda_{j}}
\end{array}\right)\right]\left(\begin{array}{c}
\hat{c}_{i,-m}^{(n+1)} \\
\vdots \\
\hat{c}_{i, n}^{(n+1)}
\end{array}\right) \\
& =\sum_{j=0}^{T-1} G_{u_{i} u_{i}}^{-1}\left(\lambda_{j}\right)\left(\begin{array}{c}
I_{y_{i} x^{K}}^{(n)}\left(\lambda_{j}\right) e^{-i m \lambda_{j}}+I_{x^{K} y_{i}}^{(n)}\left(\lambda_{j}\right) e^{i m \lambda_{j}} \\
\vdots \\
I_{y_{i} x^{K}}^{(n)}\left(\lambda_{j}\right) e^{i n \lambda_{j}}+I_{x^{K} y_{i}}^{(n)}\left(\lambda_{j}\right) e^{-i n \lambda_{j}}
\end{array}\right) .
\end{aligned}
$$


Once again, we suggest a zig-zag procedure that estimates $\mathbf{c}_{i}$. and $\psi_{i}$ for given $\boldsymbol{\theta}_{u_{i}}$ and $\boldsymbol{\theta}_{u_{i}}$ for a given $\mathbf{c}_{i}$. and $\psi_{i}$, although it is not clear that we really need to fully maximise the expected log-likelihood function at each EM iteration since the generalised EM principle simply requires us to increase it. Obviously, such iterations would be unnecessary when the idiosyncratic terms are static.

\subsection{The EM algorithm in practice}

Taking into account the theoretical results obtained in the previous sections, the step by step spectral EM algorithm combined with the iterated indirect inference procedure can be described as follows:

\section{Preliminary steps}

- Choose some arbitrary initial values for the parameters $\boldsymbol{\theta}_{x}^{(0)}, \boldsymbol{\psi}^{(0)}, \boldsymbol{\theta}_{u}^{(0)}, \mathbf{c}^{(0)}$.

- Select the number of EM iterations $M$.

- Select the number of zig-zag iterations $Z$ for the spectral Cochrane-Orcutt estimator.

- Select the maximum number of Indirect Inference iterations Q, as well as a convergence tolerance $\delta$.

- Set $\mathrm{n}=0$.

\section{EM algorithm}

1. Compute $I_{x^{K} x^{K}}^{(n)}$ and $\omega^{(n)}$ using expressions (12) and (A1), respectively (E-step).

2. Treat $I_{x^{K} x^{K}}^{(n)}+\omega^{(n)}$ as if it were the periodogram of the observed data and iterate the indirect inference procedure in section 3 until the number of iterations is equal to $Q$ or the norm of the change in the parameters is less than $\delta$. Store the results into $\boldsymbol{\theta}_{x}^{(n+1)}$ (M-step).

3. Compute first $I_{\mathbf{y} x^{K}}^{(n)}$ and then $I_{\mathbf{u u}}^{(n)}$ using expressions (13) and (14), respectively (E-step).

4. Perform $Z$ iterations of the zig-zag procedure in the following way (M-step).

(a) Treat each element $I_{u_{i} u_{i}}^{(n)}$ of the diagonal of $I_{\mathbf{u u}}^{(n)}$ as if it were the periodogram of an observed univariate series and perform the iterated indirect inference procedure in section 3 until the number of iterations is equal to $Q$ or the norm of the change in the parameters is less than $\delta$. Store the results into $\psi_{i}^{(n+1)}$ and $\theta_{u_{i}}^{(n+1)}$. 
(b) For the given value of $\theta_{u_{i}}^{(n+1)}$ solve the modified linear system of normal equations in Section 4.2 to obtain $\mathbf{c}_{i}^{(n+1)}$.

(c) Repeat (a) and (b) $Z$ times.

5. if $n \geq M$ stop, else $n=n+1$ and go to 1 .

\subsection{Convergence properties of the EM algorithm}

To assess the speed and convergence properties of our proposed spectral EM algorithm, we have generated 100 samples of size $N=10$ and $T=300$ from a generalised version of the dynamic factor model in (F8) in which both common and idiosyncratic factors follow $\operatorname{Arma}(1,1)$ processes with autoregressive parameters $\alpha_{x}=\alpha_{i}=.95$ and moving average parameters $\beta_{x}=-.5$ and $\beta_{i}=.5, i=1, \ldots, N$. These values correspond to the ones considered in section 3.3 that most closely resemble those found in the empirical application described in section 5. Note that the presence of MA components prevents the use of the time domain versions of the EM algorithm mentioned in the introduction. As for the factor loadings, we introduce some heterogeneity by assuming that they are $\mathbf{c}_{0}=(1,2,0.5,1.5,1.5,0.5,2, .5,1.5,1.5)^{\prime}$ and $\mathbf{c}_{1}=$ $(1,0.5,1.5,0.5,1.5,0.5,0.5,1.5,1.5,2)^{\prime}$. Finally, we also allow for different variances for the idiosyncratic innovations. Specifically, we consider $\gamma=(1.5,0.5,1.5,0.5,1.5,0.5,1.5,0.5,1.5,0.5)^{\prime}$, which leads to widely different signal to noise ratios across series.

We carry out two Cochrane-Orcutt iterations only within each EM iteration. Similarly, for each univariate ARMA-type maximisation we use the same convergence $L_{1}$ criterion as in section 3.3 with a maximum of five II iterations. As starting values, we assume unit loadings on the contemporaneous and lagged values of the common factor, unit specific variances, and all autoregressive and moving average coefficients set to 0.5 and 0.1 , respectively. These initial values are far away from the true parameters.

We attempt to reproduce the likely behaviour of empirical researchers by capping the number of EM iterations to 300. As in section 3.3, we also consider as benchmark the direct maximisation of the spectral log-likelihood function using the QN procedure in MATLAB's fmincon routine with the analytical first derivatives in appendix $\mathrm{C}$ and the spectral estimator of the information matrix in appendix E in lieu of the Hessian. Finally, we also look at two combined procedures that switch to QN after either 5 or 10 EM iterations. For completeness, we also look at the values of the log-likelihood at those switching points, as well as the ratio of the $L_{2}$ norm of the differences between the initial values and the values of the parameters at that point to the $L_{2}$ norm of the differences between initial and final values. Such a measure gives us a precise indication of the ground covered by the EM algorithm after a fixed number of iterations. 
The results are described in Table 2 based on 99 Monte Carlo replications. ${ }^{11}$ Given the persistence in the common factor, we re-normalise the results so that $c_{01}=1$, replacing this parameter with the variance of the innovations in the common factor, $f_{t}$. As can be seen, 300 iterations of the EM algorithm are almost as costly in terms of CPU as the direct maximisation of the spectral log-likelihood. They also fail to reach the maximum, although they get very close. However, a significant fraction of the gains in fit are achieved after just a few iterations. For that reason, the mixed methods, which converge to the maximum roughly twice as fast, provide a very good compromise. In that regard, switching after ten EM iterations slightly dominates doing so after five.

Ideally, we would like to repeat this exercise with a larger cross-sectional dimension $N$, but the computational cost of a Monte Carlo exercise involving the QN method becomes prohibitive. Nevertheless, we conjecture that the optimal number of EM iterations conducted before switching to the direct maximisation of the log-likelihood function is an increasing function of $N$.

Figure 1 illustrates a typical example of our Monte Carlo results with ten series, while Figure 2 corresponds to a model with one hundred series, with a design which is a tenfold replica of the one described in the first paragraph of this section. Remarkably, the first iteration of the EM yields a massive increase in the log-likelihood function in both cases. In addition, successive iterations also provide noticeable gains. As expected, though, the algorithm slows down considerably as we approach the optimum. Reassuringly, though, if we conduct a sufficiently large number of iterations, the value of the estimated parameters coincides with the estimates obtained by maximising the marginal log-likelihood function directly using the method of scoring with analytical expressions for the score and information matrix.

\subsection{Efficiency gains of modelling the common factors' ARMA processes}

Dynamic factor models with ARMA $(\mathrm{p}, \mathrm{q})$ processes for the common factors may alternatively be written as models with simpler $\mathrm{AR}(\mathrm{p})$ common factors by replacing the dynamic loadings polynomials $c_{i}(L)$ with $c_{i}^{*}(L)=c_{i}(L) \beta_{x}(L), i=1, \ldots, N$ (see the end of section 2.1 for a more general discussion). Therefore, one might argue that the indirect procedures described in section 3 are unnecessary, at least as far as the common factors are concerned. However, by ignoring the commonality of $\beta_{x}(L)$, the number of parameters that must be estimated increases in proportion to the number of series, which in turn may lead to substantial efficiency losses.

To assess the extent to which this potential efficiency loss is relevant in practice, we have conducted two Monte Carlo experiments with a very simple exchangeable design with 300 observations for 10 and 80 series, in which the only dynamic feature is the serial correlation of the

\footnotetext{
${ }^{11}$ In the remaining replication, the mixed procedures converged to a local maximum with a lower value for the log-likelihood than the one achieved by the EM algorithm after 5,000 iterations, which in turn coincided with the value yielded by the QN method.
} 
common factor. Specifically, we assume that $x_{t}$ follows an $\operatorname{Arma}(1,1)$ process with parameters $\alpha=.95$ and $\beta=.5$, and all the factor loadings and idiosyncratic variances are equal to 1 . We then estimate two observationally equivalent versions of this dynamic factor model. In the first one, $x_{t}$ explicitly contains a first-order MA component, which forces us to employ our proposed iterated indirect inference procedure. In the second version, in contrast, we model the common factor as an AR(1) process, but add an additional lag in the dynamic loadings of each series. Nevertheless, the static nature of the idiosyncratic terms together with the pure autoregressive nature of the common factor imply that our proposed EM algorithm is able to estimate this alternative model very quickly even for large values of $N$ without resorting to indirect inference or Cochrane-Orcutt iterations.

We assess the differences between the two procedures by looking at the MSE in estimating (i) the white noise innovations in the common factor and (ii) the common component for each series, which we compute as the difference between the observed series and the estimate of its idiosyncratic terms. Since both these quantities are common to the two representations of the dynamic factor model when evaluated at the true parameter values, their differences only reflects the sampling variability in the ML estimators (see Ansley and Kohn (1986)). Our results are based on 2,000 replications. Importantly, we average the MSE of all the time series observations except the first and last 50, so as to avoid end of sample effects. Further, we average across series whenever possible to improve the precision of our estimates.

As a benchmark, we can use the fact that at the true values the theoretical MSEs for $f_{t}$ are .8525 when $N=10$ and .6589 when $N=80$, while the respective figures for $c_{i} x_{t}=y_{i t}-u_{i t}$ are 2.95 and .8528 . When we explicitly model the common factor as an ARMA $(1,1)$ process, the MSEs for $f_{t}$ at the ML estimates become .8598 when $N=10$ and .6709 when $N=80$, so the efficiency loss resulting from parameter uncertainty is minor. In contrast, when we ignore the common nature of the MA polynomial $\beta_{x}(L)$, those MSEs become $1.0740(\mathrm{~N}=10)$ and 1.0146 $(\mathrm{N}=80)$, which are noticeably worse. This deterioration is far more acute if we look at the common components of the series. In particular, while the MSEs of $y_{i t}-u_{i t}$ are 3.7278 for $N=10$ and 1.5596 for $N=80$ when we explicitly model the common factor as an ARMA $(1,1)$ process, they become $6.0188(\mathrm{~N}=10)$ and $3.0534(\mathrm{~N}=80)$, implying deteriorations of $104.4 \%$ and $252.4 \%$ in the precision with which we estimate those components.

These results are due to a substantial efficiency loss in the estimators of the model parameters, and in particular in the factor loadings. Specifically, the interquartile range in the estimates of a typical contemporaneous loading $c_{i 0}$ goes from .26 to 1.98 when $N=10$ and from .31 to 3.10 when $N=80$, while the corresponding figures for the implied dynamic loadings are .23 versus $1.98(\mathrm{~N}=10)$ and .24 versus $3.10(\mathrm{~N}=80)$. Moreover, the median biases are also noticeably larger. In contrast, the effect on the idiosyncratic variances and especially the AR parameter of 
the common factor is much lower. Therefore, we can safely conclude that ignoring the common MA roots for the purposes of avoiding our iterated indirect inference procedures is not advisable, especially if the cross-sectional dimension is reasonably large.

\section{Common dynamics in sectoral employment}

\subsection{Dynamic factor models in practice}

There is a long tradition of analysing comovements of sectoral activity indicators (see for example Abraham and Katz (1986), Lilien (1982) or Rogerson (1987)). In this context, dynamic factor models have proved useful in assessing the extent to which observed fluctuations in sectoral aggregates are accounted for by common sources of variation. In their seminal paper, Quah and Sargent (1993) studied the behavior of annual employment series across sixty US industries over the period 1948-1989. They found that the bulk of the time variation of the different sectors was explained by a common factor, and that their estimated measure of "business activity" captured aggregate dynamics in sectoral employment very well.

Motivated by their results, we downloaded employment series from the Bureau of Labor Statistics corresponding to the 81 NAICS 3-digit sectors, measured at monthly frequency and seasonally adjusted, for the period 1990M1-2014M4, which was the longest available (see Table 3 for the list of sectors). We decided to work with (annualised) growth rates for $T=291$ months in view of the overwhelming evidence that the $(\log )$ levels of those employment series are nonstationary. ${ }^{12}$

Since our latent factor is meant to capture the common source of variation across sectoral employment growth rates, we followed Quah and Sargent (1993) and considered a dynamic single factor model. In order to determine the dynamic specification of common and specific factors, as well as the dynamic impact of the former on each sectoral series, we carried out some preliminary empirical analysis. Given that one can expect total nonfarm employment to provide an imperfect proxy for the latent variable, we fitted univariate ARMA models of various orders to the (geometric) rate of growth of this observed series. We found that an ARMA $(1,1)$ yields the best fit according to both the Schwartz and Akaike criteria. Next we regressed the demeaned changes of employment on the demeaned contemporaneous and one-month lagged changes of total nonfarm employment. We found that the coefficients associated to the lagged changes were significantly different from zero for a sizeable fraction of the series, which strongly suggests that the sector-specific employment growth rates may be driven not only by the contemporaneous

\footnotetext{
${ }^{12}$ A preliminary check on data quality indicated that a handful of series display abnormal values. We treated them as additive outliers in the (log) levels and replaced them by the average of the adjacent observations, which is a simple filter that is nevertheless optimal under the random walk hypothesis.
} 
value of the latent factor but also by its one-month lagged values. In addition, we conducted LM tests for first- and second-order residual autocorrelation to assess whether the idiosyncratic disturbances are likely to be serially correlated. We found that roughly $2 / 3$ of the series require serially correlated idiosyncratic terms.

In view of those findings, we began by estimating a special case of model (F8) in which both $x_{t}$ and $x_{t-1}$ heterogeneously affect each of the sectoral growth rates, $x_{t}$ follows an ARMA $(1,1)$ process while the idiosyncratic terms $u_{i t}$ follow simple AR(1)'s. Individual tests for $H_{0}: \alpha_{i}=0$ indicated that there are 35 series for which the white noise hypothesis is not rejected, ${ }^{13}$ which we decided to impose thenceforth. For the remaining 46 series we jointly tested the null of $\operatorname{Ar}(1)$ against ARMA $(1,1)$ specific factors, the likelihood ratio statistic taking the extremely significant value of $1369.9 .^{14}$

Estimation of the final model with $46 \operatorname{Arma}(1,1)$ and 35 white noise idiosyncratic processes was conducted by means of the EM algorithm using the iterated indirect procedure discussed in previous sections. As starting values, we assumed again unit loadings on the contemporaneous and lagged values of the common factor, unit specific variances, and autoregressive and moving average coefficients set 0.5 and 0.1 , respectively, for both common and idiosyncratic factors. As expected, the direct maximisation of the log-likelihood function in (5) starting from the same starting values failed to converge.

In order to speed up the EM iterations, we conducted five Cochrane-Orcutt iterations only instead of continuing until convergence. Despite the hundreds of parameters involved, this procedure worked very well to begin with. Eventually, though, the norm of the gradients corresponding to the idiosyncratic parameters of three series reached a positive lower bound. A careful inspection suggested that the corresponding AR and MA coefficients were probably too close to each other, and the resulting quasi cancellations slowed down our iterated indirect inference procedure, as predicted by the Monte Carlo results in section 3.3. For that reason, we switched at that point to an alternative, slower version of the EM algorithm that relied instead in the direct maximisation of the expected log-likelihood function in (16) using a scoring algorithm with analytical derivatives and information matrix. Although the estimated parameters did not change much, the log-likelihood function improved slightly and the norm of the gradients went down all the way to 0. Finally, we computed standard errors of the parameter estimators using the analytical expressions for the information matrix in appendix E. The estimation results are reported in Tables 4 and 5 .

\footnotetext{
${ }^{13}$ The series are: 581819222326283234353637383940414345474851545862636566707173 757779 81, which by and large coincide with the LM tests computed for the total nonfarm regressions.

${ }^{14}$ See Fiorentini and Sentana (2013) for computationally simple and intuitive individual and joint LM tests for neglected serial correlation in common and specific factors.
} 
As is well known, the usual Wiener-Kolmogorov filter can lead to filtering distortions at both ends of the sample. For that reason, we wrote the model in state-space form and applied the Kalman filter in the time domain with exact initial conditions derived from the stationary distribution of the 165 state variables ( 3 for the common factor and 2 for each of the idiosyncratic ones; see appendix $\mathrm{G}$ for details). ${ }^{15}$ Given that the standard fixed interval smoother was numerically unstable with such a big state vector, we used the modified Bryson-Frazier smoother instead (see Bierman (1977)). In Figure 3 we benchmark our procedure by plotting the yearly growth rate of our estimated employment factor (solid blue line) and the total nonfarm employment (red dashed line). Importantly, our smoothed factor is remarkably close to the actual growth rate of aggregate employment, especially during recession phases, such as in 1991, 2001, and 2009, although it is unsurprisingly smoother than the observed series.

\subsection{Comparison with semiparametric approaches}

One potential drawback of our parametric procedure is that it might yield misleading results if the dynamic factor model specification is incorrect. Although the testing procedures in Fiorentini and Sentana (2016b) are designed to detect those situations, the filtered estimates of the state variables are likely to be heavily influenced by the dynamic specification of the model when $N$ is relatively small.

As we mentioned in the introduction, an alternative possibility is to resort to non-parametric procedures, which impose much less structure on the spectral density matrix.

Conceptually, the simplest such procedure is the spectral version of principal components put forward by Brillinger (1981, ch. 9). The idea is to create a linear combination of the Fourier components of the observed series at a finite number of frequencies, with weights given by $\mathbf{p}_{1}(\lambda)$, which is the eigenvector associated to the largest eigenvalue of a standard non-parametric estimate of the spectral density matrix of $\mathbf{y}_{t}$ at the same frequency. The resulting spectral series is then transformed to the time domain by means of the inverse Fourier transform. We use the BUSY software developed by Fiorentini and Planas (2003), which in turn follows Forni, Hallin, Lippi and Reichlin (2000) (FHLR) in the choice of frequencies and non-parametric spectral estimator.

Unfortunately, the fact that we can multiply complex eigenvectors by a complex number on the unit circle without altering its scale implies that we can arbitrarily phase shift the spectral principal component differently for different frequencies. For that reason, we choose the scaling at

\footnotetext{
${ }^{15}$ The main difference between the Wiener-Kolmogorov filtered values, $x_{t \mid \infty}^{K}$, and the Kalman filter smoothed values, $x_{t \mid T}^{K}$, results from the implicit dependence of the former on a doubly infinite sequence of past and future observations. As shown by Fiorentini (1995) and Gómez (1999), though, they can be made numerically identical by replacing both pre- and post- sample observations by their least squares projections onto the linear span of the sample observations.
} 
each frequency in such a way that the principal component is in phase with the actual growth rate of aggregate employment. We do so by dividing $\mathbf{p}_{1}(\lambda)$ by $\mathbf{p}_{1}^{*}(\lambda) \mathbf{f}_{\mathbf{y} e}(\lambda) /\left|\mathbf{p}_{1}^{*}(\lambda) \mathbf{f}_{\mathbf{y} e}(\lambda)\right|$, where $\mathbf{f}_{\mathbf{y} e}(\lambda)$ is the cross-spectral density between the rates of growth of the 80 sectoral employment series and non-farm employment, so that the co-spectrum between this re-scaled principal component and the rate of growth of aggregate employment is frequency-invariant.

FHLR considered an alternative way of constructing an index of comovements. Specifically, they cross-sectionally averaged the component attributable to the common factor for each of the series by the spectral principal component, which is univocally defined regardless of the normalisation chosen for $\mathbf{p}_{1}(\lambda)$. Given the widely different employment levels across sectors, we consider a weighted average that reflects relative sector sizes at each point in time.

The two additional indices are depicted in Figure 4, together with our estimated factor. The dynamic principal component is the noisiest of the three measures, which is probably due to the phase normalisation that we have chosen. The FHLR index is also more erratic than the estimate of the common factor produced by the Kalman filter smoother, but sometimes it leads it, providing a more timely indication of turning. This suggests that adding "forward" loadings to our dynamic factor model constitutes an interesting avenue for research. Overall, though, our smoothed factor provides a clearer signal, with a slightly higher correlation with total nonfarm employment.

\section{Directions for further work}

The spectral EM algorithm developed in the previous sections can be extended in several interesting directions. One obvious possibility would be models with multiple common factors. Although this would be intensive in notation, such an extension would be otherwise straightforward after dealing with identification issues before estimating the model. However, sometimes it would be necessary to add many common factors to adequately capture the off-diagonal elements of the autocovariance matrices even though the number of pervasive sources of variation is small. To some extent, this fact explains the success of approximate factor structures. In those situations in which it is natural to group the $N$ series into $R$ homogeneous blocks, an attractive alternative solution are bifactor models with two types of factors:

1. Pervasive common factors that affect all $N$ series

2. Block factors that only affect a subset of the series, such as the ones belonging to the same country or region. 
In Fiorentini, Galesi and Sentana (2016) we explain how to efficiently exploit the sparsity of the factor loading matrix of bifactor models so that the spectral EM algorithm we propose in this paper can successfully estimate them by maximum likelihood with a large number of series from multiple regions. In this regard, it would be convenient to extend our algorithm to dynamic trifactor models, in which each block has a bifactor structure of its own. Such models would be particularly well suited to the analysis of international business cycles using a large set of country specific macro variables.

More generally, we could in principle extend our numerical procedures to dynamic factor structures with non-diagonal idiosyncratic spectral density matrices because in those models the factorisation of the complete log-likelihood function of observed series and common factors will still be true. Nevertheless, except in models with contemporaneously correlated, white noise idiosyncratic terms, we would have to resort to frequency domain versions of multivariate regressions, whose numerically efficient estimation when $N$ is relatively large deserves further consideration. For that reason, an extension of the Doz, Giannone and Reichlin (2012) analysis that looks at the properties of our algorithm and the resulting ML estimators in approximate factor models in which the cross-sectional dimension is non-negligible relative to the time series dimension would constitute a very valuable addition. In fact, a very large number of series might constitute a computational blessing in this framework, the rationale being that for large $N$ the unobservable factors will be consistently estimated by the Kalman-Wiener-Kolmogorov filter, so that the model effectively becomes a multivariate regression model.

Another interesting extension would deal with models in which the heterogeneous dynamic impact of the common factor on each observed variable, which is characterised by the $c_{i}(L)$ polynomials, can be represented by the ratio of two low order polynomials (see Hannan (1965) and Hannan and Nichols (1972) for frequency domain estimators of some rational distributed lag models when $x_{t}$ is observable).

Finally, it is worth mentioning that although we have exploited some specificities of dynamic factor models, our procedures can be easily extended to most unobserved components time series processes, and in particular to UCARIMA models and the state-space models underlying the recent nowcasting literature (see Fiorentini and Sentana (2016a) and Banbura, Giannone and Reichlin (2011), respectively, and the references therein). We are currently pursuing some of these research avenues. 


\section{References}

Abraham, K. G. and Katz, L. F. (1986): "Cyclical unemployment: sectoral shifts or aggregate disturbances", Journal of Political Economy 94, 507-522.

Arcidiacono, P. and Jones, J.B. (2003): "Finite mixture distributions, sequential likelihood and the EM algorithm", Econometrica 71, 933-946.

Ansley, C.F. and Kohn, R. (1986): "Prediction mean squared error for state space models with estimated parameters", Biometrika 73, 467-473.

Bai, J. and Ng, S. (2008): "Large dimensional factor analysis", Foundations and trends in econometrics 3, 89-163.

Banbura, M., Giannone, D., and Reichlin, L. (2011): "Nowcasting", in M.P. Clemens and D.F. Hendry (eds.), Oxford Handbook on Economic Forecasting, 193-224, Oxford University Press.

Barndorff-Nielsen, O. and Schou, G. (1973): "On the parametrization of autoregressive models by partial autocorrelations", Journal of Multivariate Analysis 3, 408-419.

Bergstrom, A.R. (1984): "Continuous time stochastic models and issues of aggregation over time ", chapter 20 in Z. Griliches \& M. D. Intriligator (eds.), Handbook of Econometrics II, 1145-1212, North-Holland.

Bierman, G.J. (1977) Factorization methods for discrete sequential estimation, Academic Press.

Brillinger, D. R. (1981): Time series: data analysis and theory, Holt, Rinehart and Winston.

Chamberlain, G. and Rothschild, M. (1983): “Arbitrage, factor structure, and mean-variance analysis on large asset markets", Econometrica 51, 1281-1304.

Chambers, M.J. and Thorton, M.A. (2012): "Discrete time representation of continuous time Arma processes", Econometric Theory 28, 219-238.

Choudhuri , N., Ghosal S. and Roy, A. (2004): "Contiguity of the Whittle measure for a Gaussian time series", Biometrika 91, 211-218.

Chumacero, R. (2001): "Estimating ARma models efficiently", Studies in Nonlinear Dynamics and Econometrics 5, 1-14.

Cochrane, D. and Orcutt, G.H. (1949): “Application of least squares regression to relationships containing auto-correlated error terms", Journal of the American Statistical Association $44,32-61$.

Demos, A., and Sentana, E. (1998): "An EM algorithm for conditionally heteroskedastic factor models", Journal of Business and Economic Statistics 16, 357-361. 
Dempster, A., Laird, N., and Rubin, D. (1977): "Maximum likelihood from incomplete data via the EM algorithm", Journal of the Royal Statistical Society B 39, 1-38.

Diebold, F.X. and Rudebusch, G.D. (1996), "Measuring business cycles: a modern perspective", Review of Economics and Statistics, 78, 67-77.

Diebold, F.X., Rudebusch, G.D. and Aruoba, B. (2006), "The macroeconomy and the yield curve: a dynamic latent factor approach", Journal of Econometrics 131, 309-338.

Doz, C., Giannone, D. and Reichlin, L. (2012): "A quasi maximum likelihood approach for large approximate dynamic factor models", Review of Economics and Statistics 94, 1014-1024.

Dungey, M., Martin, V.L. and Pagan, A.R. (2000): "A multivariate latent factor decomposition of international bond yield spreads", Journal of Applied Econometrics 15, 697-715.

Dunsmuir, W. (1979): "A central limit theorem for parameter estimation in stationary vector time series and its application to models for a signal observed with noise", Annals of Statistics 7, 490-506.

Dunsmuir, W. and Hannan, E.J. (1976): "Vector linear time series models", Advances in Applied Probability 8, 339-364.

Engle, R.F., Hendry, D.F. and Richard, J.-F. (1983): "Exogeneity", Econometrica, 277-304.

Engle, R.F. and Watson, M.W. (1981): "A one-factor multivariate time series model of metropolitan wage rates", Journal of the American Statistical Association 76, 774-781.

Fiorentini, G. (1995): Conditional heteroskedasticity: some results on estimation, inference and signal extraction, with an application to seasonal adjustment, unpublished Doctoral Dissertation, European University Institute.

Fiorentini, G., Galesi, A. and Sentana (2016): "Fast ML estimation of dynamic bifactor models: an application to European inflation", in S.J. Koopman and E.T. Hillebrand (eds.) Dynamic Factor Models, Advances in Econometrics 35, 215-282, Emerald.

Fiorentini, G. and Planas, C. (2003): Busy Programme User Manual: Tools and Practices for Business Cycle Analysis in the European Union. Joint Research Centre of European Commission http://ipsc.jrc.ec.europa.eu/fileadmin/repository/sfa/finepro/software/BUSY-manual0603.pdf

Fiorentini, G. and Sentana, E. (2016a): "Neglected serial correlation tests in UCARImA models", SERIES 7, 121-178.

Fiorentini, G. and Sentana, E. (2016b): "Dynamic specification tests for dynamic factor models", mimeo, CEMFI.

Fiorentini, G., Sentana, E. and Shephard, N. (2004): "Likelihood estimation of latent generalised ARCH structures", Econometrica 72, 1481-1517.

Forni, M., Hallin, M., Lippi, M. and Reichlin, L. (2000): "The generalized dynamic factor model: identification and estimation", Review of Economics and Statistics 82, 540-554. 
Forni, M., Hallin, M., Lippi, M. and Zaffaroni, P. (2015): "Dynamic factor models with infinite-dimensional factor spaces: one-sided representations", Journal of Econometrics 185, 359-371.

Forni, M. and Lippi, M. (2001): "The generalized dynamic factor model: representation theory", Econometric Theory 17, 1113-1141.

Forni, M. and Lippi, M. (2011): "The general dynamic factor model: one-sided representation results", Journal of Econometrics 163, 23-28.

Fruhwirth-Schnatter, S. (2007): Finite mixture and Markov switching models, Springer.

Geweke, J.F. (1977): "The dynamic factor analysis of economic time series models", in D. Aigner and A. Goldberger (eds.), Latent variables in socioeconomic models, 365-383, NorthHolland.

Geweke, J.F. and Singleton, K.J (1981): "Maximum likelihood "confirmatory" factor analysis of economic time series", International Economic Review 22, 37-54.

Ghysels, E., Khalaf, L. and Vodounou, C. (2003): "Simulation based inference in moving average models", Annales d'Economie et de Statistique 69, 85-99.

Gómez, V. (1999): "Three equivalent methods for filtering finite nonstationary time series", Journal of Business and Economic Statistics 17, 109-116.

Gouriéroux, C., Monfort, A. and Renault, E. (1991): "A general framework for factor models", mimeo, INSEE.

Gouriéroux, C., Monfort, A. and Renault, E. (1993): "Indirect inference", Journal of Applied Econometrics 8, S85-S118.

Gregory, A.W., Head, A.C. and Raynauld, J. (1997): "Measuring world business cycles", International Economic Review 38, 677-701.

Hamilton, J. (1990): "Analysis of time series subject to changes in regime", Journal of Econometrics 45, 39-70

Hannan, E.J. (1965): "The estimation of relationships involving distributed lags", Econometrica 33, 206-224.

Hannan, E.J. (1969): "The estimation of mixed moving average autoregressive systems", Biometrika 56, 579-593.

Hannan, E.J. and Nicholls, D.F. (1972): "The estimation of mixed regression, autoregression, moving average, and distributed lag models", Econometrica 40, 529-547.

Harvey, A.C. (1989): Forecasting, structural models and the Kalman filter, Cambridge University Press.

Heaton, C. and Solo, V. (2004): "Identification of causal factor models of stationary time series", Econometrics Journal, 7, 618-627. 
Jegadeesh, N. and G.G. Pennacchi (1996): "The behavior of interest rates implied by the term structure of Eurodollar futures", Journal of Money, Credit and Banking 28, 426-446.

Jungbacker, B. and S.J. Koopman (2015): "Likelihood-based dynamic factor analysis for measurement and forecasting", Econometrics Journal 17, 1-21.

Lawley, D.N. and Maxwell, A.E. (1971): Factor analysis as a statistical method, 2nd ed., Butterworths.

Lehmann, B.N., and Modest, D.M. (1988): "The empirical foundations of the arbitrage pricing theory ", Journal of Financial Economics 21, 213-254.

Lilien, D. M. (1982): "Sectoral shifts and cyclical unemployment", Journal of Political Economy 90, 777-793.

Litterman, R. and Sargent, T.J. (1979): "Detecting neutral price level changes and the effects of aggregate demand with index models", Research Department Working Paper 125, Federal Reserve Bank of Minneapolis.

Louis, T. A. (1982): "Finding observed information using the EM algorithm", Journal of the Royal Statistical Society B, 44, 98-103.

McLachlan, G. and Krishnan, T. (1996): The EM algorithm and extensions. Wiley.

Magnus, J.R. (1988): Linear structures, Oxford University Press.

Magnus, J.R. and Neudecker, H. (1988): Matrix differential calculus with applications in Statistics and Econometrics, Wiley.

Maravall, A. (1993): "Stochastic linear trends: models and estimators", Journal of Econometrics 56, 5-37.

Mody, A. and Taylor, M.P. (2007): "Regional vulnerability: the case of East Asia", Journal of International Money and Finance 26, 1292-1310.

Peña, D. and Box, G.E.P. (1987): "Identifying a simplifying structure in time series", Journal of the American Statistical Association 82, 836-843.

Quah, D. and Sargent, T. (1993): "A dynamic index model for large cross sections", in Stock, J.H. and Watson, M.W. (eds) Business cycles, indicators and forecasting, 285-310, University of Chicago Press.

Rogerson, R. (1987): "An equilibrium model of sectoral reallocation", Journal of Political Economy 95, 824-834.

Rubin, D. and Thayer, D. (1982): "EM algorithms for ML factor analysis", Psychometrika 47, 69-76.

Ruud, P. (1991): "Extensions of estimation methods using the EM algorithm", Journal of Econometrics 49, 305-341. 
Sargent, T.J., and Sims, C.A. (1977): "Business cycle modeling without pretending to have too much a priori economic theory", New methods in business cycle research 1, 145-168.

Sentana, E. (2000): "The likelihood function of conditionally heteroskedastic factor models", Annales d'Economie et de Statistique 58, 1-19.

Sentana, E. (2004): "Factor representing portfolios in large asset markets", Journal of Econometrics 119, 257-289.

Sentana, E., Calzolari, G. and Fiorentini, G. (2008): "Indirect estimation of large conditionally heteroskedastic factor models, with an application to the Dow 30 stocks", Journal of Econometrics 146, 10-25.

Shumway, R., and D. Stoffer (1982): "An approach to time series smoothing and forecasting using the EM algorithm", Journal of Time Series Analysis 3, 253-264.

Singleton, K.J. (1981): "A latent time series model of the cyclical behavior of interest rates", International Economic Review 21, 559-575.

Stock, J.H. and Watson, M.W. (1989): "New indexes of coincident and leading economic indicators", NBER Macroeconomics Annual 4, 351-394.

Stock, J., and Watson, M. (1991), "A probability model of the coincident economic indicators", in K. Lahiri and G. Moore (eds.) Leading economic indicators: new approaches and forecasting records, Cambridge University Press.

Stock, J.H. and Watson, M.W. (1993): "A procedure for predicting recessions with leading indicators: econometric issues and recent experience", in J.H. Stock and M.W. Watson (eds.) Business cycles, indicators, and forecasting, 95-153, University of Chicago Press.

Tanner, M. (1996): Tools for statistical inference: methods for the exploration of posterior distributions and likelihood functions (3rd ed.), Springer-Verlag.

Watson, M.W. and Engle, R.F. (1983): "Alternative algorithms for estimation of dynamic MIMIC, factor, and time varying coefficient regression models", Journal of Econometrics 23, 385-400.

Watson, M.W. and Kraft, D.F. (1984): "Testing the interpretation of indices in a macroeconomic index model", Journal of Monetary Economics 13, 165-182.

Whittle, P. (1962): "Gaussian estimation in stationary time series", Bulletin of the International Statistical Institute 39, 105-129. 


\section{Appendices}

\section{A The Woodbury formula}

Computations can be considerably speeded up by exploiting the Woodbury formula under the assumption that neither $G_{x x}(\lambda)$ nor $\mathbf{G}_{\mathbf{u u}}(\lambda)$ are singular at any frequency (see Sentana (2000) for a generalisation). Specifically, we obtain

$$
\begin{aligned}
\left|\mathbf{G}_{\mathbf{y y}}(\lambda)\right| & =\left|\mathbf{G}_{\mathbf{u u}}(\lambda)\right| G_{x x}(\lambda) \omega(\lambda) \\
\mathbf{G}_{\mathbf{y y}}^{-1}(\lambda) & =\mathbf{G}_{\mathbf{u u}}^{-1}(\lambda)-\omega(\lambda) \mathbf{G}_{\mathbf{u u}}^{-1}(\lambda) \mathbf{c}\left(e^{-i \lambda}\right) \mathbf{c}^{\prime}\left(e^{i \lambda}\right) \mathbf{G}_{\mathbf{u u}}^{-1}(\lambda), \\
\omega(\lambda) & =\left[G_{x x}^{-1}(\lambda)+\mathbf{c}^{\prime}\left(e^{i \lambda}\right) \mathbf{G}_{\mathbf{u u}}^{-1}(\lambda) \mathbf{c}\left(e^{-i \lambda}\right)\right]^{-1}
\end{aligned}
$$

The advantage of these expressions is that $\mathbf{G}_{\mathbf{u u}}(\lambda)$ is a diagonal matrix and $\omega(\lambda)$ a scalar, which greatly simplifies the computations. On this basis, the transfer function of the WienerKolmogorov common factor smoother becomes

$$
G_{x x}(\lambda) \mathbf{c}^{\prime}\left(e^{i \lambda}\right) \mathbf{G}_{\mathbf{y y}}^{-1}(\lambda)=\omega(\lambda) \mathbf{c}^{\prime}\left(e^{i \lambda}\right) \mathbf{G}_{\mathbf{u u}}^{-1}(\lambda),
$$

so

$$
G_{x^{K} x^{K}}(\lambda)=\omega(\lambda) G_{x x}(\lambda) \mathbf{c}^{\prime}\left(e^{i \lambda}\right) \mathbf{G}_{\mathbf{u u}}^{-1}(\lambda) \mathbf{c}\left(e^{-i \lambda}\right)=G_{x x}(\lambda)-\omega(\lambda),
$$

where we have used the fact that

$$
\omega(\lambda) \mathbf{c}^{\prime}\left(e^{i \lambda}\right) \mathbf{G}_{\mathbf{u u}}^{-1}(\lambda) \mathbf{c}\left(e^{-i \lambda}\right)=1-\omega(\lambda) G_{x x}^{-1}(\lambda),
$$

which can be easily proved by dividing both sides by $\omega(\lambda)$.

Similarly, the transfer function of the Wiener-Kolmogorov specific factors smoother will be

$$
\mathbf{G}_{\mathbf{u u}}(\lambda) \mathbf{G}_{\mathbf{y y}}^{-1}(\lambda)=\mathbf{I}_{N}-\omega(\lambda) \mathbf{c}\left(e^{-i \lambda}\right) \mathbf{c}^{\prime}\left(e^{i \lambda}\right) \mathbf{G}_{\mathbf{u u}}^{-1}(\lambda)
$$

so

$$
\mathbf{G}_{\mathbf{u}^{K} \mathbf{u}^{K}}(\lambda)=\mathbf{G}_{\mathbf{u u}}(\lambda)-\omega(\lambda) \mathbf{c}\left(e^{-i \lambda}\right) \mathbf{c}^{\prime}\left(e^{i \lambda}\right) .
$$

Finally,

$$
\mathbf{G}_{x^{K} \mathbf{u}^{K}}(\lambda)=\omega(\lambda) \mathbf{c}^{\prime}\left(e^{i \lambda}\right)
$$

We can also exploit the same formula to compute the quadratic form $\mathbf{z}_{j}^{\mathbf{y} *} \mathbf{G}_{\mathbf{y y}}^{-1}\left(\lambda_{j}\right) \mathbf{z}_{j}^{\mathbf{y}}$ as

$$
\begin{gathered}
\mathbf{z}_{j}^{\mathbf{y} *} \mathbf{G}_{\mathbf{u u}}^{-1}\left(\lambda_{j}\right) \mathbf{z}_{j}^{\mathbf{y}}-\mathbf{z}_{j}^{\mathbf{y} *} \mathbf{G}_{\mathbf{u u}}^{-1}(\lambda) \omega\left(\lambda_{j}\right) \mathbf{c}\left(e^{-i \lambda}\right) \mathbf{c}^{\prime}\left(e^{i \lambda}\right) \mathbf{G}_{\mathbf{u u}}^{-1}(\lambda) \mathbf{z}_{j}^{\mathbf{y}} \\
=\mathbf{z}_{j}^{\mathbf{y} *} \mathbf{G}_{\mathbf{u u}}^{-1}\left(\lambda_{j}\right) \mathbf{z}_{j}^{\mathbf{y}}-\omega\left(\lambda_{j}\right) z_{j}^{x^{K} *}(\boldsymbol{\theta}) z_{j}^{x^{K}}(\boldsymbol{\theta})
\end{gathered}
$$

where

$$
z_{j}^{x^{K}}(\boldsymbol{\theta})=E\left[z_{j}^{x} \mid \mathbf{Z}^{\mathbf{y}}, \boldsymbol{\theta}\right]=G_{x x}\left(\lambda_{j}\right) \mathbf{c}^{\prime}\left(e^{i \lambda_{j}}\right) \mathbf{G}_{\mathbf{y y}}^{-1}\left(\lambda_{j}\right) \mathbf{z}_{j}^{\mathbf{y}}=\omega(\lambda) \mathbf{c}^{\prime}\left(e^{i \lambda_{j}}\right) \mathbf{G}_{\mathbf{u u}}^{-1}\left(\lambda_{j}\right) \mathbf{z}_{j}^{\mathbf{y}}
$$

denotes the filtered value of $z_{j}^{x}$ given the observed series and the current $\boldsymbol{\theta}$ from (3). 


\section{B The minimal sufficient statistics for $\left\{x_{t}\right\}$}

In any given realisation of the vector process $\left\{\mathbf{y}_{t}\right\}$, the values of $\left\{x_{t}\right\}$ could be regarded as a set of $T$ parameters. With this interpretation in mind, we can define $x_{t \mid \infty}^{G}$ as the spectral GLS estimator of $x_{t}$ through the transformation

$$
\left.d Z^{x^{G}}(\lambda)=\left[\mathbf{c}^{\prime}\left(e^{i \lambda}\right) \mathbf{G}_{\mathbf{u u}}^{-1}(\lambda) \mathbf{c}\left(e^{-i \lambda}\right)\right]^{-1} \mathbf{c}^{\prime i \lambda}\right) \mathbf{G}_{\mathbf{u u}}^{-1}(\lambda) d \mathbf{Z}^{\mathbf{y}}(\lambda) .
$$

Similarly, we can define $\mathbf{u}_{t \mid \infty}^{G}$ through

$$
d \mathbf{Z}^{\mathbf{u}^{G}}(\lambda)=\left\{\mathbf{I}_{N}-\left[\mathbf{c}^{\prime}\left(e^{i \lambda}\right) \mathbf{G}_{\mathbf{u u}}^{-1}(\lambda) \mathbf{c}\left(e^{-i \lambda}\right)\right]^{-1} \mathbf{c}^{\prime}\left(e^{i \lambda}\right) \mathbf{G}_{\mathbf{u u}}^{-1}(\lambda)\right\} d \mathbf{Z}^{\mathbf{y}}(\lambda) .
$$

It is then easy to see that the joint spectral density of $x_{t \mid \infty}^{G}$ and $\mathbf{u}_{t \mid \infty}^{G}$ will be block-diagonal, with the $(1,1)$ element being

$$
G_{x x}(\lambda)+\left[\mathbf{c}^{\prime}\left(e^{i \lambda}\right) \mathbf{G}_{\mathbf{u u}}^{-1}(\lambda) \mathbf{c}\left(e^{-i \lambda}\right)\right]^{-1}
$$

and the $(2,2)$ block

$$
\mathbf{G}_{\mathbf{y y}}(\lambda)-\mathbf{c}\left(e^{-i \lambda}\right)\left[\mathbf{c}^{\prime}\left(e^{i \lambda}\right) \mathbf{G}_{\mathbf{u u}}^{-1}(\lambda) \mathbf{c}\left(e^{-i \lambda}\right)\right]^{-1} \mathbf{c}^{\prime}\left(e^{i \lambda}\right),
$$

whose rank is $N-1$. This orthogonalisation allows us to factorise the spectral log-likelihood function of $\mathbf{y}_{t}$ as the sum of the log-likelihood function of $x_{t \mid \infty}^{G}$, which is univariate, and the log-likelihood function of $\mathbf{u}_{t \mid \infty}^{G}$. The Jacobian of the transformation is 1 , as we can write

$$
\begin{aligned}
& \left(\begin{array}{c}
{\left[\mathbf{c}^{\prime}\left(e^{i \lambda}\right) \mathbf{G}_{\mathbf{u u}}^{-1}(\lambda) \mathbf{c}\left(e^{-i \lambda}\right)\right]^{-1} \mathbf{c}^{\prime}\left(e^{i \lambda}\right) \mathbf{G}_{\mathbf{u u}}^{-1}(\lambda)} \\
\left\{\mathbf{I}_{N}-\mathbf{c}\left(e^{-i \lambda}\right)\left[\mathbf{c}^{\prime}\left(e^{i \lambda}\right) \mathbf{G}_{\mathbf{u u}}^{-1}(\lambda) \mathbf{c}\left(e^{-i \lambda}\right)\right]^{-1} \mathbf{c}^{\prime}\left(e^{i \lambda}\right) \mathbf{G}_{\mathbf{u u}}^{-1}(\lambda)\right\}
\end{array}\right)=\left(\begin{array}{cc}
1 & 0 \\
0 & \mathbf{G}_{\mathbf{u u}}^{1 / 2}(\lambda)
\end{array}\right) \\
& \times\left(\begin{array}{c}
{\left[\mathbf{c}^{\prime}\left(e^{i \lambda}\right) \mathbf{G}_{\mathbf{u u}}^{-1}(\lambda) \mathbf{c}\left(e^{-i \lambda}\right)\right]^{-1} \mathbf{c}^{\prime}\left(e^{i \lambda}\right) \mathbf{G}_{\mathbf{u u}}^{-1 / 2}(\lambda)} \\
\left\{\mathbf{I}_{N}-\mathbf{G}_{\mathbf{u u}}^{-1 / 2}(\lambda) \mathbf{c}\left(e^{-i \lambda}\right)\left[\mathbf{c}^{\prime}\left(e^{i \lambda}\right) \mathbf{G}_{\mathbf{u u}}^{-1}(\lambda) \mathbf{c}\left(e^{-i \lambda}\right)\right]^{-1} \mathbf{c}^{\prime}\left(e^{i \lambda}\right) \mathbf{G}_{\mathbf{u u}}^{-1 / 2}(\lambda)\right\}
\end{array}\right) \mathbf{G}_{\mathbf{u u}}^{-1 / 2}(\lambda),
\end{aligned}
$$

where the matrix in the centre is orthogonal. Importantly, the parameters characterising $G_{x x}(\lambda)$ only enter through the first component. In contrast, the remaining parameters affect both components. Moreover, we can easily show that

1. $x_{t \mid T}^{G}=x_{t}+\zeta_{t \mid T}^{G}$, with $x_{t}$ and $\zeta_{t \mid T}^{G}$ orthogonal at all leads and lags.

2. The smoothed estimator of $x_{t}$ obtained by applying the Wiener- Kolmogorov filter to $x_{t \mid \infty}^{G}$ coincides with $x_{t \mid \infty}^{K}$.

This confirms that $x_{t \mid \infty}^{G}$ constitute minimal sufficient statistics for $x_{t}$, thereby generalising earlier results by Jungbacker and Koopman (2015), who considered models in which $\mathbf{c}\left(e^{-i \lambda}\right)=\mathbf{c}$ for all $\lambda$, and Fiorentini, Sentana and Shephard (2004), who looked at the related class of factor models with time-varying volatility (see also Gouriéroux, Monfort and Renault (1991)). In addition, the degree of unobservability of $x_{t}$ depends exclusively on the size of $\left[\mathbf{c}^{\prime}\left(e^{i \lambda}\right) \mathbf{G}_{\mathbf{u u}}^{-1}(\lambda) \mathbf{c}\left(e^{-i \lambda}\right)\right]^{-1}$ relative to $G_{x x}(\lambda)$ (see Sentana (2004) for a closely related discussion). 


\section{Spectral scores}

The score function for all the parameters other than the mean is given by (6). Since

$$
d \mathbf{G}_{\mathbf{y y}}(\lambda)=d \mathbf{c}\left(e^{-i \lambda}\right) G_{x x}(\lambda) \mathbf{c}^{\prime}\left(e^{i \lambda}\right)+\mathbf{c}\left(e^{-i \lambda}\right) d G_{x x}(\lambda) \mathbf{c}^{\prime}\left(e^{i \lambda}\right)+\mathbf{c}\left(e^{-i \lambda}\right) G_{x x}(\lambda) d \mathbf{c}^{\prime}\left(e^{i \lambda}\right)+d \mathbf{G}_{\mathbf{u u}}(\lambda)
$$

(see Magnus and Neudecker (1988)), it immediately follows that

$$
\begin{aligned}
& \operatorname{dvec}\left[\mathbf{G}_{\mathbf{y y}}(\lambda)\right]=\left[\mathbf{c}\left(e^{i \lambda}\right) G_{x x}(\lambda) \otimes \mathbf{I}_{N}\right] d \mathbf{c}\left(e^{-i \lambda}\right)+\left[\mathbf{I}_{N} \otimes \mathbf{c}\left(e^{-i \lambda}\right) G_{x x}(\lambda)\right] d \mathbf{c}\left(e^{i \lambda}\right) \\
&+\left[\mathbf{c}\left(e^{i \lambda}\right) \otimes \mathbf{c}\left(e^{-i \lambda}\right)\right] d G_{x x}(\lambda)+\mathbf{E}_{N} d v e c d\left[\mathbf{G}_{\mathbf{u u}}(\lambda)\right]
\end{aligned}
$$

where $\mathbf{E}_{N}^{\prime}=\left(\mathbf{e}_{1} \mathbf{e}_{1}^{\prime}|\ldots| \mathbf{e}_{N} \mathbf{e}_{N}^{\prime}\right)$, with $\left(\mathbf{e}_{1}|\ldots| \mathbf{e}_{N}\right)=\mathbf{I}_{N}$, is the unique $N^{2} \times N$ "diagonalisation" matrix that transforms $\operatorname{vec}(\mathbf{A})$ into $\operatorname{vecd}(\mathbf{A})$ as $\operatorname{vecd}(\mathbf{A})=\mathbf{E}_{N}^{\prime} \operatorname{vec}(\mathbf{A})$, and $\mathbf{K}_{m n}$ is the commutation matrix of orders $m$ and $n$ (see Magnus (1988)). But

$$
\mathbf{c}\left(e^{-i \lambda}\right)=\sum_{l=-m}^{n} \mathbf{c}_{l}(\boldsymbol{\theta}) e^{-i l \lambda}
$$

so

$$
d \mathbf{c}\left(e^{-i \lambda}\right)=\sum_{l=-m}^{n} d \mathbf{c}_{l}(\boldsymbol{\theta}) e^{-i l \lambda}
$$

Consequently, we can write

$$
\begin{aligned}
\operatorname{dvec}\left[\mathbf{G}_{\mathbf{y y}}(\lambda)\right]= & \sum_{l=-m}^{n}\left\{\left[e^{-i l \lambda} \mathbf{c}\left(e^{i \lambda}\right) G_{x x}(\lambda) \otimes \mathbf{I}_{N}\right]+\left[\mathbf{I}_{N} \otimes e^{i l \lambda} \mathbf{c}\left(e^{-i \lambda}\right) G_{x x}(\lambda)\right]\right\} d \mathbf{c}_{l}(\boldsymbol{\theta}) \\
& +\left[\mathbf{c}\left(e^{i \lambda}\right) \otimes \mathbf{c}\left(e^{-i \lambda}\right)\right] d G_{x x}(\lambda)+\mathbf{E}_{N} d v e c d\left[\mathbf{G}_{\mathbf{u u}}(\lambda)\right] .
\end{aligned}
$$

Hence, the Jacobian of $\operatorname{vec}\left[\mathbf{G}_{\mathbf{y y}}(\lambda)\right]$ will be

$$
\begin{aligned}
\frac{\partial v e c\left[\mathbf{G}_{\mathbf{y y}}(\lambda)\right]}{\partial \boldsymbol{\theta}^{\prime}}= & \sum_{l=-m}^{n}\left\{\begin{array}{c}
{\left[e^{-i l \lambda} \mathbf{c}\left(e^{i \lambda}\right) G_{x x}(\lambda) \otimes \mathbf{I}_{N}\right]} \\
+\left[\mathbf{I}_{N} \otimes e^{i l \lambda} \mathbf{c}\left(e^{-i \lambda}\right) G_{x x}(\lambda)\right]
\end{array}\right\} \frac{\partial \mathbf{c}_{l}}{\partial \boldsymbol{\theta}^{\prime}} \\
& +\left[\mathbf{c}\left(e^{i \lambda}\right) \otimes \mathbf{c}\left(e^{-i \lambda}\right)\right] \frac{\partial G_{x x}(\lambda)}{\partial \boldsymbol{\theta}^{\prime}}+\mathbf{E}_{N} \frac{\partial v e c d\left[\mathbf{G}_{\mathbf{u u}}(\lambda)\right]}{\partial \boldsymbol{\theta}^{\prime}} .
\end{aligned}
$$

If we combine this expression with the fact that

$$
\begin{aligned}
& {\left[\mathbf{G}_{\mathbf{y y}}^{-1}\left(\lambda_{j}\right) \otimes \mathbf{G}_{\mathbf{y y}}^{\prime-1}\left(\lambda_{j}\right)\right] \operatorname{vec}\left[\mathbf{z}_{j}^{\mathbf{y} c} \mathbf{z}_{j}^{\mathbf{y} \prime}-\mathbf{G}_{\mathbf{y y}}^{\prime}\left(\lambda_{j}\right)\right] } \\
= & \operatorname{vec}\left[2 \pi \mathbf{G}_{\mathbf{y y}}^{\prime-1}\left(\lambda_{j}\right) \mathbf{z}_{j}^{\mathbf{y} c} \mathbf{z}_{j}^{\mathbf{y} \prime} \mathbf{G}_{\mathbf{y y}}^{\prime-1}\left(\lambda_{j}\right)-\mathbf{G}_{\mathbf{y y}}^{\prime-1}\left(\lambda_{j}\right)\right]
\end{aligned}
$$


and $\mathbf{I}_{\mathbf{y y}}^{\prime}\left(\lambda_{j}\right)=\mathbf{z}_{j}^{\mathbf{y c}} \mathbf{z}_{j}^{\mathbf{y} \prime}$ we obtain:

$$
\begin{gathered}
\begin{array}{c}
\mathbf{d}(\lambda ; \boldsymbol{\theta})=\sum_{l=-m}^{n} \frac{\partial \mathbf{c}_{l}^{\prime}}{\partial \boldsymbol{\theta}}\left\{\begin{array}{c}
{\left[e^{-i l \lambda} G_{x x}(\lambda) \mathbf{c}^{\prime}\left(e^{i \lambda}\right) \otimes \mathbf{I}_{N}\right]} \\
+\left[\mathbf{I}_{N} \otimes e^{i l \lambda} G_{x x}(\lambda) \mathbf{c}^{\prime}\left(e^{-i \lambda}\right)\right]
\end{array}\right\} v e c\left[2 \pi \mathbf{G}_{\mathbf{y y}}^{\prime-1}(\lambda) \mathbf{I}_{\mathbf{y y}}^{\prime}(\lambda) \mathbf{G}_{\mathbf{y y}}^{\prime-1}(\lambda)-\mathbf{G}_{\mathbf{y y}}^{\prime-1}(\lambda)\right] \\
+\frac{\partial G_{x x}(\lambda)}{\partial \boldsymbol{\theta}}\left[\mathbf{c}^{\prime}\left(e^{i \lambda}\right) \otimes \mathbf{c}^{\prime}\left(e^{-i \lambda}\right)\right] v e c\left[2 \pi \mathbf{G}_{\mathbf{y y}}^{\prime-1}(\lambda) \mathbf{I}_{\mathbf{y y}}^{\prime}(\lambda) \mathbf{G}_{\mathbf{y y}}^{\prime-1}(\lambda)-\mathbf{G}_{\mathbf{y y}}^{\prime-1}(\lambda)\right] \\
+\frac{\partial v e c d^{\prime}\left[\mathbf{G}_{\mathbf{u u}}(\lambda)\right]}{\partial \boldsymbol{\theta}} \mathbf{E}_{N} v e c\left[2 \pi \mathbf{G}_{\mathbf{y y}}^{\prime-1}(\lambda) \mathbf{I}_{\mathbf{y y}}^{\prime}(\lambda) \mathbf{G}_{\mathbf{y y}}^{\prime-1}(\lambda)-\mathbf{G}_{\mathbf{y y}}^{\prime-1}(\lambda)\right] \\
=\sum_{l=-m}^{n} \frac{\partial \mathbf{c}_{l}^{\prime}}{\partial \boldsymbol{\theta}} v e c\left[\begin{array}{c}
2 \pi \mathbf{G}_{\mathbf{y y}}^{\prime-1}(\lambda) \mathbf{I}_{\mathbf{y y}}^{\prime}(\lambda) \mathbf{G}_{\mathbf{y y}}^{\prime-1}(\lambda) \mathbf{c}\left(e^{i \lambda}\right) G_{x x}(\lambda) e^{-i l \lambda}-\mathbf{G}_{\mathbf{y y}}^{\prime-1}(\lambda) \mathbf{c}\left(e^{i \lambda}\right) G_{x x}(\lambda) e^{-i l \lambda} \\
+2 \pi e^{i l \lambda} G_{x x}(\lambda) \mathbf{c}^{\prime}\left(e^{-i \lambda}\right) \mathbf{G}_{\mathbf{y y}}^{\prime-1}(\lambda) \mathbf{I}_{\mathbf{y y}}^{\prime}(\lambda) \mathbf{G}_{\mathbf{y y}}^{\prime-1}(\lambda)-e^{i l \lambda} G_{x x}(\lambda) \mathbf{c}^{\prime}\left(e^{-i \lambda}\right) \mathbf{G}_{\mathbf{y y}}^{\prime-1}(\lambda)
\end{array}\right] \\
+\frac{\partial G_{x x}(\lambda)}{\partial \boldsymbol{\theta}} \operatorname{vec}\left[2 \pi \mathbf{c}^{\prime}\left(e^{-i \lambda}\right) \mathbf{G}_{\mathbf{y y}}^{\prime-1}(\lambda) \mathbf{I}_{\mathbf{y y}}^{\prime}(\lambda) \mathbf{G}_{\mathbf{y y}}^{\prime-1}(\lambda) \mathbf{c}\left(e^{i \lambda}\right)-\mathbf{c}^{\prime}\left(e^{-i \lambda}\right) \mathbf{G}_{\mathbf{y y}}^{\prime-1}(\lambda) \mathbf{c}\left(e^{i \lambda}\right)\right] \\
+\frac{\partial v e c d^{\prime}\left[\mathbf{G}_{\mathbf{u u}}(\lambda)\right]}{\partial \boldsymbol{\theta}} \mathbf{E}_{N} v e c\left[2 \pi \mathbf{G}_{\mathbf{y y}}^{\prime-1}(\lambda) \mathbf{I}_{\mathbf{y y}}^{\prime}(\lambda) \mathbf{G}_{\mathbf{y y}}^{\prime-1}(\lambda)-\mathbf{G}_{\mathbf{y y}}^{\prime-1}(\lambda)\right]
\end{array}
\end{gathered}
$$

Let us now try to interpret the different components of this expression. The first thing to note is that

$$
e^{-i l \lambda} v e c\left[2 \pi \mathbf{G}_{\mathbf{y y}}^{\prime-1}(\lambda) \mathbf{I}_{\mathbf{y y}}^{\prime}(\lambda) \mathbf{G}_{\mathbf{y y}}^{\prime-1}(\lambda) \mathbf{c}\left(e^{i \lambda}\right) G_{x x}(\lambda)-\mathbf{G}_{\mathbf{y y}}^{\prime-1}(\lambda) \mathbf{c}\left(e^{i \lambda}\right) G_{x x}(\lambda)\right]
$$

and

$$
e^{i l \lambda} v e c\left[2 \pi G_{x x}(\lambda) \mathbf{c}^{\prime}\left(e^{-i \lambda}\right) \mathbf{G}_{\mathbf{y y}}^{\prime-1}(\lambda) \mathbf{I}_{\mathbf{y y}}^{\prime}(\lambda) \mathbf{G}_{\mathbf{y y}}^{\prime-1}(\lambda)-G_{x x}(\lambda) \mathbf{c}^{\prime}\left(e^{-i \lambda}\right) \mathbf{G}_{\mathbf{y y}}^{\prime-1}(\lambda)\right]
$$

are complex conjugates because $\mathbf{G}_{\mathbf{y y}}(\lambda)$ is Hermitian and the conjugate of a product is the product of the conjugates, so it suffices to analyse one of them.

If we further assume that $G_{x x}(\lambda)>0$ and $\mathbf{G}_{\mathbf{u u}}(\lambda)>\mathbf{0}$ we can write

$$
\begin{gathered}
2 \pi \mathbf{G}_{\mathbf{y y}}^{\prime-1}(\lambda) \mathbf{I}_{\mathbf{y y}}^{\prime}(\lambda) \mathbf{G}_{\mathbf{y y}}^{\prime-1}(\lambda) \mathbf{c}\left(e^{i \lambda}\right) G_{x x}(\lambda)-\mathbf{G}_{\mathbf{y y}}^{\prime-1}(\lambda) \mathbf{c}\left(e^{i \lambda}\right) G_{x x}(\lambda) \\
=\mathbf{G}_{\mathbf{u u}}^{\prime-1}(\lambda)\left[2 \pi \mathbf{I}_{x^{K} \mathbf{u}^{K}}^{\prime}(\lambda)-\mathbf{G}_{x^{K} \mathbf{u}^{K}}^{\prime}(\lambda)\right] \\
2 \pi \mathbf{c}^{\prime}\left(e^{-i \lambda}\right) \mathbf{G}_{\mathbf{y y}}^{\prime-1}(\lambda) \mathbf{I}_{\mathbf{y y}}^{\prime}(\lambda) \mathbf{G}_{\mathbf{y y}}^{\prime-1}(\lambda) \mathbf{c}\left(e^{i \lambda}\right)-\mathbf{c}^{\prime}\left(e^{-i \lambda}\right) \mathbf{G}_{\mathbf{y y}}^{\prime-1}(\lambda) \mathbf{c}\left(e^{i \lambda}\right) \\
=G_{x x}^{-2}(\lambda)\left[2 \pi I_{x^{K} x^{K}}(\lambda)-G_{x^{K} x^{K}}(\lambda)\right]
\end{gathered}
$$

and

$$
2 \pi \mathbf{G}_{\mathbf{y y}}^{\prime-1}(\lambda) \mathbf{I}_{\mathbf{y y}}^{\prime}(\lambda) \mathbf{G}_{\mathbf{y y}}^{\prime-1}(\lambda)-\mathbf{G}_{\mathbf{y y}}^{\prime-1}(\lambda)=\mathbf{G}_{\mathbf{u u}}^{\prime-1}(\lambda)\left[2 \pi \mathbf{I}_{\mathbf{u}^{K} \mathbf{u}^{K}}^{\prime}(\lambda)-\mathbf{G}_{\mathbf{u}^{K} \mathbf{u}^{K}}^{\prime}(\lambda)\right] \mathbf{G}_{\mathbf{u u}}^{\prime-1}(\lambda)
$$

Therefore, the component of the score associated to $\mathbf{c}_{l}$ will be the sum across frequencies of terms of the form

$$
e^{-i l \lambda} \mathbf{G}_{\mathbf{u u}}^{\prime-1}(\lambda)\left[2 \pi \mathbf{I}_{x^{K} \mathbf{u}^{K}}^{\prime}(\lambda)-\mathbf{G}_{x^{K} \mathbf{u}^{K}}^{\prime}(\lambda)\right]
$$

(and their conjugate transposes), which capture the difference between the cross-periodogram and cross-spectrum of $x_{t-l}^{K}$ and $u_{i t}^{K}$ inversely weighted by the spectral density of $u_{i t}$. As a result, 
we can understand this term as arising from the normal equation in the spectral regression of $y_{i t}$ onto $x_{t-l}$ but taking into account the unobservability of the regressor.

Similarly, the component of the score associated to the parameters that determine $G_{x x}(\lambda)$ will be the cross-product across frequencies of the product of the derivatives of the spectral density of $x_{t}$ with the difference between the periodogram and spectrum of $x_{t}^{K}$ inversely weighted by the squared spectral density of $x_{t}$. In this case, we can interpret this term as arising from a marginal log-likelihood function for $x_{t}$ that takes into account the unobservability of $x_{t}$.

Finally, the component of the score associated to the parameters in $G_{u_{i} u_{i}}(\lambda)$ will be the crossproduct across frequencies of the product of the derivatives of the spectral density of $u_{i t}$ with the difference between the periodogram and spectrum of $u_{i t}^{K}$ inversely weighted by the squared spectral density of $u_{i t}$. Once again, we can interpret this term as arising from the conditional log-likelihood function of $u_{i t}$ given $x_{t}$ that takes into account the unobservability of $u_{t_{i}}$.

As usual, we can then exploit the Woodbury formula, as in expressions (12), (D6) and (D7), to greatly speed up the computations. In particular, we will get

$$
\begin{gathered}
G_{x x}(\lambda) \mathbf{c}^{\prime}\left(e^{i \lambda}\right) \mathbf{G}_{\mathbf{y y}}^{-1}(\lambda) \mathbf{I}_{\mathbf{y y}}(\lambda) \mathbf{G}_{\mathbf{y y}}^{-1}(\lambda)-G_{x x}(\lambda) \mathbf{c}^{\prime}\left(e^{i \lambda}\right) \mathbf{G}_{\mathbf{y y}}^{-1}(\lambda) \\
=G_{x x}(\lambda) \mathbf{c}^{\prime}\left(e^{i \lambda}\right) \mathbf{G}_{\mathbf{y y}}^{-1}(\lambda) \mathbf{I}_{\mathbf{y y}}(\lambda)\left[\mathbf{G}_{\mathbf{u u}}^{-1}(\lambda)-\omega(\lambda) \mathbf{G}_{\mathbf{u u}}^{-1}(\lambda) \mathbf{c}\left(e^{-i \lambda}\right) \mathbf{c}^{\prime}\left(e^{i \lambda}\right) \mathbf{G}_{\mathbf{u u}}^{-1}(\lambda)\right]-\omega(\lambda) \mathbf{c}^{\prime}\left(e^{i \lambda}\right) \mathbf{G}_{\mathbf{u u}}^{-1}(\lambda) \\
=G_{x x}(\lambda) \mathbf{c}^{\prime}\left(e^{i \lambda}\right) \mathbf{G}_{\mathbf{y y}}^{-1}(\lambda) \mathbf{I}_{\mathbf{y y}}(\lambda)\left[\mathbf{I}_{N}-\omega(\lambda) \mathbf{G}_{\mathbf{u u}}^{-1}(\lambda) \mathbf{c}\left(e^{-i \lambda}\right) \mathbf{c}^{\prime}\left(e^{i \lambda}\right)\right] \mathbf{G}_{\mathbf{u u}}^{-1}(\lambda)-\omega(\lambda) \mathbf{c}^{\prime}\left(e^{i \lambda}\right) \mathbf{G}_{\mathbf{u u}}^{-1}(\lambda) \\
=\left[\mathbf{I}_{x^{K} \mathbf{u}^{K}}(\lambda)-\omega(\lambda) \mathbf{c}^{\prime}\left(e^{i \lambda}\right)\right] \mathbf{G}_{\mathbf{u u}}^{-1}(\lambda), \\
=\left[\mathbf{G}_{\mathbf{u u}}^{-1}(\lambda)-\omega(\lambda) \mathbf{G}_{\mathbf{u u}}^{-1}(\lambda) \mathbf{c}\left(e^{-i \lambda}\right) \mathbf{c}^{\prime}\left(e^{i \lambda}\right) \mathbf{G}_{\mathbf{u u}}^{-1}(\lambda)\right] \mathbf{I}_{\mathbf{y y}}(\lambda)\left[\mathbf{G}_{\mathbf{u u}}^{-1}(\lambda)-\omega(\lambda) \mathbf{G}_{\mathbf{u u}}^{-1}(\lambda) \mathbf{c}\left(e^{-i \lambda}\right) \mathbf{c}^{\prime}\left(e^{i \lambda}\right) \mathbf{G}_{\mathbf{u u}}^{-1}(\lambda)\right] \\
-\left[\mathbf{G}_{\mathbf{u u}}^{-1}(\lambda)-\omega(\lambda) \mathbf{G}_{\mathbf{u u}}^{-1}(\lambda) \mathbf{c}\left(e^{-i \lambda}\right) \mathbf{c}^{\prime}\left(e^{i \lambda}\right) \mathbf{G}_{\mathbf{u u}}^{-1}(\lambda)\right] \\
\mathbf{G}_{\mathbf{u u}}^{-1}(\lambda)\left[\mathbf{I}_{N}-\omega(\lambda) \mathbf{c}\left(e^{-i \lambda}\right) \mathbf{c}^{\prime}\left(e^{i \lambda}\right) \mathbf{G}_{\mathbf{u u}}^{-1}(\lambda)\right] \mathbf{I}_{\mathbf{y y}}(\lambda)\left[\mathbf{I}_{N}-\omega(\lambda) \mathbf{G}_{\mathbf{u u}}^{-1}(\lambda) \mathbf{c}\left(e^{-i \lambda}\right) \mathbf{c}^{\prime}\left(e^{i \lambda}\right)\right] \mathbf{G}_{\mathbf{u u}}^{-1}(\lambda) \\
-\mathbf{G}_{\mathbf{u u}}^{-1}(\lambda)\left[\mathbf{G}_{\mathbf{u u}}(\lambda)-\omega(\lambda) \mathbf{c}\left(e^{-i \lambda}\right) \mathbf{c}^{\prime}\left(e^{i \lambda}\right)\right] \mathbf{G}_{\mathbf{u u}}^{-1}(\lambda) \\
=\mathbf{G}_{\mathbf{u u}}^{-1}(\lambda)\left[\mathbf{I}_{\mathbf{u}^{K} \mathbf{u}^{K}}(\lambda)-\mathbf{G}_{\mathbf{u}^{K} \mathbf{u}^{K}}(\lambda)\right] \mathbf{G}_{\mathbf{u u}}^{-1}(\lambda),
\end{gathered}
$$

and

$\mathbf{c}^{\prime}\left(e^{i \lambda}\right) \mathbf{G}_{\mathbf{y y}}^{-1}(\lambda) \mathbf{I}_{\mathbf{y y}}(\lambda) \mathbf{G}_{\mathbf{y y}}^{-1}(\lambda) \mathbf{c}\left(e^{-i \lambda}\right)-\mathbf{c}^{\prime}\left(e^{i \lambda}\right) \mathbf{G}_{\mathbf{y y}}^{-1}(\lambda) \mathbf{c}\left(e^{-i \lambda}\right)=G_{x x}^{-1}(\lambda)\left[I_{x^{K} x^{K}}(\lambda)-G_{x^{K} x^{K}}(\lambda)\right] G_{x x}^{-1}(\lambda)$.

\section{Alternative marginal scores}

As is well known, the EM algorithm slows down considerably near the optimum (see e.g. Tanner (1996)). At that point, the best practical strategy would be to switch to a first derivativebased method. In this regard, the EM principle can also be exploited to simplify the computation 
of the score This result was first noted by Louis (1982); see also Ruud (1991) and Tanner (1996). Since the Kullback inequality implies that $E[l(\mathbf{x} \mid \mathbf{Y} ; \boldsymbol{\theta}) \mid \mathbf{Y} ; \boldsymbol{\theta}]=0$, it is clear that $\partial l(\mathbf{Y} ; \boldsymbol{\theta}) / \partial \boldsymbol{\theta}$ can be obtained as the expected value (given $\mathbf{Y}$ and $\boldsymbol{\theta}$ ) of the sum of the unobservable scores corresponding to $l\left(\mathbf{y}_{1}, \ldots, \mathbf{y}_{N} \mid \mathbf{x}\right)$ and $l(\mathbf{x})$. This yields

$$
\begin{aligned}
\frac{\partial l(\mathbf{Y})}{\partial \boldsymbol{\theta}_{x}} & =\frac{1}{2} \sum_{j=0}^{T-1} \frac{\partial G_{x x}\left(\lambda_{j}\right)}{\partial \boldsymbol{\theta}_{x}} G_{x x}^{-2}\left(\lambda_{j}\right)\left[2 \pi E\left[z_{j}^{x} z_{j}^{x *} \mid \mathbf{Z}_{y}, \boldsymbol{\theta}\right]-G_{x x}\left(\lambda_{j}\right)\right] \\
\frac{\partial l(\mathbf{Y})}{\partial \boldsymbol{\theta}_{u_{i}}} & =\frac{1}{2} \sum_{j=0}^{T-1} \frac{\partial G_{u_{i} u_{i}}\left(\lambda_{j}\right)}{\partial \boldsymbol{\theta}_{u_{i}}} G_{u_{i} u_{i}}^{-2}\left(\lambda_{j}\right)\left[2 \pi E\left[z_{j}^{u_{i}} z_{j}^{u_{i} *} \mid \mathbf{Z}_{y}, \boldsymbol{\theta}\right]-G_{u_{i} u_{i}}\left(\lambda_{j}\right)\right] \\
\frac{\partial l(\mathbf{Y})}{\partial c_{i, k}} & =\frac{2 \pi}{2} \sum_{j=0}^{T-1} G_{u_{i} u_{i}}^{-1}\left(\lambda_{j}\right)\left[e^{i k \lambda_{j}} E\left[z_{j}^{u_{i}} z_{j}^{x *} \mid \mathbf{Z}_{y}, \boldsymbol{\theta}\right]+e^{-i k \lambda_{j}} E\left[z_{j}^{x} z_{j}^{u_{i} *} \mid \mathbf{Z}_{y}, \boldsymbol{\theta}\right]\right] .
\end{aligned}
$$

But since the scores are now evaluated at the values of the parameters at which the expectations are computed, we will have that

$$
\begin{aligned}
E\left[z_{j}^{x} z_{j}^{x *} \mid \mathbf{Z}^{\mathbf{y}}, \boldsymbol{\theta}\right] & =I_{x^{K} x^{K}}\left(\lambda_{j}\right)+\omega\left(\lambda_{j}\right), \\
E\left[\mathbf{z}_{j}^{\mathbf{u}} \mathbf{z}_{j}^{\mathbf{u}} \mid \mathbf{Z}^{\mathbf{y}}, \boldsymbol{\theta}\right] & =E\left[\mathbf{z}_{j}^{\mathbf{u}} \mid \mathbf{Z}^{\mathbf{y}}, \boldsymbol{\theta}\right] E\left[\mathbf{z}_{j}^{\mathbf{u} *} \mid \mathbf{Z}^{\mathbf{y}}, \boldsymbol{\theta}\right]+E\left[\left\{\mathbf{z}_{j}^{\mathbf{u}}-E\left[\mathbf{z}_{j}^{\mathbf{u}} \mid \mathbf{Z}^{\mathbf{y}}, \boldsymbol{\theta}\right]\right\}\left\{\mathbf{z}_{j}^{\mathbf{u} *}-E\left[\mathbf{z}_{j}^{\mathbf{u} *} \mid \mathbf{Z}^{\mathbf{y}}, \boldsymbol{\theta}\right]\right\} \mid \mathbf{Z}^{\mathbf{y}}, \boldsymbol{\theta}\right] \\
& =I_{\mathbf{u}^{K} \mathbf{u}^{K}}\left(\lambda_{j}\right)+\mathbf{c}\left(e^{-i \lambda_{j}}\right) \omega\left(\lambda_{j}\right) \mathbf{c}^{\prime}\left(e^{i \lambda_{j}}\right) . \\
E\left[\mathbf{z}_{j}^{\mathbf{u}} z_{j}^{x *} \mid \mathbf{Z}^{\mathbf{y}}, \boldsymbol{\theta}\right] & =E\left[\mathbf{z}_{j}^{\mathbf{u}} \mid \mathbf{Z}^{\mathbf{y}}, \boldsymbol{\theta}\right] E\left[z_{j}^{x *} \mid \mathbf{Z}^{\mathbf{y}}, \boldsymbol{\theta}\right]+E\left[\left\{\mathbf{z}_{j}^{\mathbf{u}}-E\left[\mathbf{z}_{j}^{\mathbf{u}} \mid \mathbf{Z}^{\mathbf{y}}, \boldsymbol{\theta}\right]\right\}\left\{z_{j}^{x *}-E\left[z_{j}^{x *} \mid \mathbf{Z}^{\mathbf{y}}, \boldsymbol{\theta}\right]\right\} \mid \mathbf{Z}^{\mathbf{y}}, \boldsymbol{\theta}\right] \\
& =I_{\mathbf{u}^{K} x^{K}}\left(\lambda_{j}\right)-\mathbf{c}\left(e^{-i \lambda_{j}}\right) \omega\left(\lambda_{j}\right)
\end{aligned}
$$

where

$$
\begin{aligned}
& z_{j}^{\mathbf{u}^{K}}=E\left[z_{j}^{\mathbf{u}} \mid \mathbf{Z}^{\mathbf{y}}, \boldsymbol{\theta}\right]=\mathbf{G}_{\mathbf{u u}}\left(\lambda_{j}\right) \mathbf{G}_{\mathbf{y y}}^{-1}\left(\lambda_{j}\right) \mathbf{z}_{j}^{\mathbf{y}}=\mathbf{z}_{j}^{\mathbf{y}}-\mathbf{c}\left(e^{-i \lambda_{j}}\right) z_{j}^{x^{K}}, \\
& E\left[\left(\mathbf{z}_{j}^{\mathbf{u}}-\mathbf{z}_{j}^{\mathbf{u}^{K}}\right)\left(\mathbf{z}_{j}^{\mathbf{u} *}-\mathbf{z}_{j}^{\mathbf{u}^{K} *}\right) \mid \mathbf{Z}^{\mathbf{y}}, \boldsymbol{\theta}\right]=\mathbf{c}\left(e^{-i \lambda_{j}}\right) \omega\left(\lambda_{j}\right) \mathbf{c}^{\prime}\left(e^{i \lambda_{j}}\right), \\
& E\left[\left(\mathbf{z}_{j}^{\mathbf{u}}-\mathbf{z}_{j}^{\mathbf{u}^{K}}\right)\left(z_{j}^{x *}-z_{j}^{x^{K} *}\right) \mid \mathbf{Z}^{\mathbf{y}}, \boldsymbol{\theta}\right]=\mathbf{c}\left(e^{-i \lambda_{j}}\right) \omega\left(\lambda_{j}\right), \\
&=2 \pi\left[\mathbf{I}_{N}-\omega\left(\lambda_{j}\right) \mathbf{c}\left(e^{-i \lambda_{j}}\right) \mathbf{c}^{\prime}\left(e^{i \lambda_{j}}\right) \mathbf{G}_{\mathbf{u u}}^{-1}\left(\lambda_{j}\right)\right] \mathbf{I}_{\mathbf{y y}}\left(\lambda_{j}\right)\left[\mathbf{I}_{N}-\omega\left(\lambda_{j}\right) \mathbf{c}\left(e^{i \lambda_{j}}\right) \mathbf{c}^{\prime}\left(e^{-i \lambda_{j}}\right) \mathbf{G}_{\mathbf{u u}}^{-1}\left(\lambda_{j}\right)\right]
\end{aligned}
$$

is the periodogram of the smoothed values of the specific factors, and

$$
\begin{aligned}
\mathbf{I}_{x^{K}} \mathbf{u}^{K}\left(\lambda_{j}\right) & =2 \pi G_{x x}\left(\lambda_{j}\right) \mathbf{c}^{\prime}\left(e^{i \lambda_{j}}\right) \mathbf{G}_{\mathbf{y y}}^{-1}\left(\lambda_{j}\right) \mathbf{I}_{\mathbf{y y}}\left(\lambda_{j}\right) \mathbf{G}_{\mathbf{y y}}^{-1}\left(\lambda_{j}\right) \mathbf{G}_{\mathbf{u u}}\left(\lambda_{j}\right) \\
& =2 \pi \omega\left(\lambda_{j}\right) \mathbf{c}^{\prime}\left(e^{i \lambda_{j}}\right) \mathbf{G}_{\mathbf{u u}}^{-1}\left(\lambda_{j}\right) \mathbf{I}_{\mathbf{y y}}\left(\lambda_{j}\right)\left[\mathbf{I}_{N}-\omega\left(\lambda_{j}\right) \mathbf{G}_{\mathbf{u u}}^{-1}\left(\lambda_{j}\right) \mathbf{c}\left(e^{-i \lambda_{j}}\right) \mathbf{c}^{\prime}\left(e^{i \lambda_{j}}\right)\right]
\end{aligned}
$$

is the co-periodogram between $x_{t \mid \infty}^{K}$ and $\mathbf{u}_{t \mid \infty}^{K}$.

Tedious algebra shows that these scores coincide with the expressions in appendix C. They also closely related to the scores of the expected log-likelihoods in section 4.2 , but the difference is that the expectations were taken there with respect to the conditional distribution of $\mathbf{x}$ given $\mathbf{Y}$ evaluated at $\boldsymbol{\theta}^{(n)}$, not $\boldsymbol{\theta}$. 


\section{E Spectral information matrix}

Given the expression for the Jacobian matrix (C5), we will have that

$$
\begin{aligned}
\frac{\partial v e c^{\prime}\left[\mathbf{G}_{\mathbf{y y}}(\lambda)\right]}{\partial \boldsymbol{\theta}}= & \sum_{l=-m}^{n} \frac{\partial \mathbf{c}_{l}^{\prime}}{\partial \boldsymbol{\theta}}\left\{\begin{array}{c}
{\left[e^{-i l \lambda} G_{x x}(\lambda) \mathbf{c}^{\prime}\left(e^{i \lambda}\right) \otimes \mathbf{I}_{N}\right]} \\
+\left[\mathbf{I}_{N} \otimes e^{i l \lambda} \mathbf{c}^{\prime}\left(e^{-i \lambda}\right) G_{x x}(\lambda)\right]
\end{array}\right\} \\
& +\frac{\partial G_{x x}(\lambda)}{\partial \boldsymbol{\theta}}\left[\mathbf{c}^{\prime}\left(e^{i \lambda}\right) \otimes \mathbf{c}^{\prime}\left(e^{-i \lambda}\right)\right]+\frac{\partial v e c d\left[\mathbf{G}_{\mathbf{u u}}(\lambda)\right]}{\partial \boldsymbol{\theta}} \mathbf{E}_{N}^{\prime}
\end{aligned}
$$

and

$$
\begin{aligned}
\left\{\frac{\partial v e c^{\prime}\left[\mathbf{G}_{\mathbf{y y}}(\lambda)\right]}{\partial \boldsymbol{\theta}}\right\}^{*}= & \sum_{l=-m}^{n}\left\{\begin{array}{c}
{\left[e^{i l \lambda} \mathbf{c}\left(e^{-i \lambda}\right) G_{x x}(\lambda) \otimes \mathbf{I}_{N}\right]} \\
+\left[\mathbf{I}_{N} \otimes e^{-i l \lambda} \mathbf{c}\left(e^{i \lambda}\right) G_{x x}(\lambda)\right]
\end{array}\right\} \frac{\partial \mathbf{c}_{l}}{\partial \boldsymbol{\theta}^{\prime}} \\
& +\left[\mathbf{c}\left(e^{-i \lambda}\right) \otimes \mathbf{c}\left(e^{i \lambda}\right)\right] \frac{\partial G_{x x}(\lambda)}{\partial \boldsymbol{\theta}^{\prime}}+\mathbf{E}_{N} \frac{\partial v e c d\left[\mathbf{G}_{\mathbf{u u}}(\lambda)\right]}{\partial \boldsymbol{\theta}^{\prime}}
\end{aligned}
$$

Hence, it is straightforward to see that the elements of the block of the information matrix

(7) corresponding to the dynamic factor loadings will be

$$
\begin{aligned}
& \frac{\partial v e c^{\prime}\left[\mathbf{G}_{\mathbf{y y}}(\lambda)\right]}{\partial \mathbf{c}_{l}}\left[\mathbf{G}_{\mathbf{y y}}^{-1}(\lambda) \otimes \mathbf{G}_{\mathbf{y y}}^{\prime-1}(\lambda)\right]\left\{\frac{\partial v e c^{\prime}\left[\mathbf{G}_{\mathbf{y y}}(\lambda)\right]}{\partial \mathbf{c}_{k}}\right\}^{*} \\
= & \left\{\begin{array}{c}
{\left[e^{-i l \lambda} G_{x x}(\lambda) \mathbf{c}^{\prime}\left(e^{i \lambda}\right) \otimes \mathbf{I}_{N}\right]} \\
+\left[\mathbf{I}_{N} \otimes e^{i l \lambda} G_{x x}(\lambda) \mathbf{c}^{\prime}\left(e^{-i \lambda}\right)\right]
\end{array}\right\}\left[\mathbf{G}_{\mathbf{y y}}^{-1}(\lambda) \otimes \mathbf{G}_{\mathbf{y y}}^{\prime-1}(\lambda)\right]\left\{\begin{array}{c}
{\left[e^{i k \lambda} \mathbf{c}\left(e^{-i \lambda}\right) G_{x x}(\lambda) \otimes \mathbf{I}_{N}\right]} \\
+\left[\mathbf{I}_{N} \otimes e^{-i k \lambda} \mathbf{c}\left(e^{i \lambda}\right) G_{x x}(\lambda)\right]
\end{array}\right\} \\
= & G_{x x}^{2}(\lambda)\left\{\begin{array}{c}
e^{-i(l+k) \lambda} \mathbf{G}_{\mathbf{y y}}^{\prime-1}(\lambda) \mathbf{c}\left(e^{i \lambda}\right) \mathbf{c}^{\prime}\left(e^{i \lambda}\right) \mathbf{G}_{\mathbf{y y}}^{-1}(\lambda) \\
+e^{i(l+k) \lambda}\left[\mathbf{G}_{\mathbf{y y}}^{-1}(\lambda) \mathbf{c}\left(e^{-i \lambda}\right) \mathbf{c}^{\prime}\left(e^{-i \lambda}\right) \mathbf{G}_{\mathbf{y y}}^{\prime-1}(\lambda)\right] \\
\left.e^{-i(l-k) \lambda} \mathbf{c}^{\prime}\left(e^{i \lambda}\right) \mathbf{G}_{\mathbf{y y}}^{-1}(\lambda) \mathbf{c}\left(e^{-i \lambda}\right)\right] \mathbf{G}_{\mathbf{y y}}^{\prime-1}(\lambda) \\
e^{i(l-k) \lambda}\left[\mathbf{c}^{\prime}\left(e^{-i \lambda}\right) \mathbf{G}_{\mathbf{y y}}^{\prime-1}(\lambda) \mathbf{c}\left(e^{i \lambda}\right)\right] \mathbf{G}_{\mathbf{y y}}^{-1}(\lambda)
\end{array}\right\}
\end{aligned}
$$

Notice that since the information matrix is real, there will be cancellation between the complex parts of the above matrices.

Similarly,

$$
\begin{aligned}
& \frac{\partial v e c^{\prime}\left[\mathbf{G}_{\mathbf{y y}}(\lambda)\right]}{\partial \mathbf{c}_{l}}\left[\mathbf{G}_{\mathbf{y y}}^{-1}(\lambda) \otimes \mathbf{G}_{\mathbf{y y}}^{\prime-1}(\lambda)\right] \frac{\partial v e c\left[\mathbf{G}_{\mathbf{y y}}(\lambda)\right]}{\partial \boldsymbol{\theta}_{x}^{\prime}} \\
= & \left\{\begin{array}{c}
{\left[e^{-i l \lambda} G_{x x}(\lambda) \mathbf{c}^{\prime}\left(e^{i \lambda}\right) \otimes \mathbf{I}_{N}\right]} \\
+\left[\mathbf{I}_{N} \otimes e^{i l \lambda} G_{x x}(\lambda) \mathbf{c}^{\prime}\left(e^{-i \lambda}\right)\right]
\end{array}\right\}\left[\mathbf{G}_{\mathbf{y y}}^{-1}(\lambda) \otimes \mathbf{G}_{\mathbf{y y}}^{\prime-1}(\lambda)\right]\left[\mathbf{c}\left(e^{-i \lambda}\right) \otimes \mathbf{c}\left(e^{i \lambda}\right)\right] \frac{\partial G_{x x}(\lambda)}{\partial \boldsymbol{\theta}_{x}^{\prime}} \\
= & G_{x x}(\lambda)\left\{\begin{array}{c}
{\left[e^{-i l \lambda} \mathbf{c}^{\prime}\left(e^{i \lambda}\right) \mathbf{G}_{\mathbf{y y}}^{-1}(\lambda) \mathbf{c}\left(e^{-i \lambda}\right)\right] \mathbf{G}_{\mathbf{y y}}^{\prime-1}(\lambda) \mathbf{c}\left(e^{i \lambda}\right)} \\
+\left[e^{i k \lambda} \mathbf{c}^{\prime}\left(e^{-i \lambda}\right) \mathbf{G}_{\mathbf{y y}}^{\prime-1}(\lambda) \mathbf{c}\left(e^{i \lambda}\right)\right] \mathbf{G}_{\mathbf{y y}}^{-1}(\lambda) \mathbf{c}\left(e^{-i \lambda}\right)
\end{array}\right\} \frac{\partial G_{x x}(\lambda)}{\partial \boldsymbol{\theta}_{x}^{\prime}},
\end{aligned}
$$

which again will be real.

In addition

$$
\begin{aligned}
& \frac{\partial v e c^{\prime}\left[\mathbf{G}_{\mathbf{y y}}(\lambda)\right]}{\partial \mathbf{c}_{l}}\left[\mathbf{G}_{\mathbf{y y}}^{-1}(\lambda) \otimes \mathbf{G}_{\mathbf{y y}}^{\prime-1}(\lambda)\right] \frac{\partial v e c\left[\mathbf{G}_{\mathbf{y y}}(\lambda)\right]}{\partial \boldsymbol{\theta}_{u_{j}}^{\prime}} \\
= & \left\{\begin{array}{c}
{\left[e^{-i l \lambda} G_{x x}(\lambda) \mathbf{c}^{\prime}\left(e^{i \lambda}\right) \otimes \mathbf{I}_{N}\right]} \\
+\left[\mathbf{I}_{N} \otimes e^{i l \lambda} G_{x x}(\lambda) \mathbf{c}^{\prime}\left(e^{-i \lambda}\right)\right]
\end{array}\right\}\left[\mathbf{G}_{\mathbf{y y}}^{-1}(\lambda) \otimes \mathbf{G}_{\mathbf{y y}}^{\prime-1}(\lambda)\right] \mathbf{E}_{N}\left(\mathbf{e}_{j} \frac{\partial G_{u_{j} u_{j}}(\lambda)}{\partial \boldsymbol{\theta}_{u_{j}}^{\prime}}\right) \\
= & G_{x x}(\lambda)\left\{\begin{array}{c}
{\left[e^{-i l \lambda} \mathbf{c}^{\prime}\left(e^{i \lambda}\right) \mathbf{G}_{\mathbf{y y}}^{-1}(\lambda) \mathbf{e}_{j}\right] \mathbf{G}_{\mathbf{y y}}^{\prime-1}(\lambda) \mathbf{e}_{j}} \\
+\left[e^{i l \lambda} \mathbf{c}^{\prime}\left(e^{-i \lambda}\right) \mathbf{G}_{\mathbf{y y}}^{\prime-1}(\lambda) \mathbf{e}_{j}\right] \mathbf{G}_{\mathbf{y y}}^{-1}(\lambda) \mathbf{e}_{j}
\end{array}\right\} \frac{\partial G_{u_{j} u_{j}}(\lambda)}{\partial \boldsymbol{\theta}_{u_{j}}^{\prime}}
\end{aligned}
$$


since

$$
\mathbf{e}_{j}^{\prime} \mathbf{E}_{N}^{\prime}=\mathbf{e}_{j}^{\prime}\left(\mathbf{e}_{1} \mathbf{e}_{1}^{\prime}|\ldots| \mathbf{e}_{N} \mathbf{e}_{N}^{\prime}\right)=\mathbf{e}_{j}^{\prime} \otimes \mathbf{e}_{j}^{\prime}
$$

In turn,

$$
\begin{aligned}
& \frac{\partial v e c^{\prime}\left[\mathbf{G}_{\mathbf{y y}}(\lambda)\right]}{\partial \boldsymbol{\theta}_{x}}\left[\mathbf{G}_{\mathbf{y y}}^{-1}(\lambda) \otimes \mathbf{G}_{\mathbf{y y}}^{\prime-1}(\lambda)\right] \frac{\partial v e c\left[\mathbf{G}_{\mathbf{y y}}(\lambda)\right]}{\partial \boldsymbol{\theta}_{x}^{\prime}} \\
= & \frac{\partial G_{x x}(\lambda)}{\partial \boldsymbol{\theta}_{x}}\left[\mathbf{c}^{\prime}\left(e^{i \lambda}\right) \otimes \mathbf{c}^{\prime}\left(e^{-i \lambda}\right)\right]\left[\mathbf{G}_{\mathbf{y y}}^{-1}(\lambda) \otimes \mathbf{G}_{\mathbf{y y}}^{\prime-1}(\lambda)\right]\left[\mathbf{c}\left(e^{-i \lambda}\right) \otimes \mathbf{c}\left(e^{i \lambda}\right)\right] \frac{\partial G_{x x}(\lambda)}{\partial \boldsymbol{\theta}_{x}^{\prime}} \\
= & {\left[\mathbf{c}^{\prime}\left(e^{i \lambda}\right) \mathbf{G}_{\mathbf{y y}}^{-1}(\lambda) \mathbf{c}\left(e^{-i \lambda}\right)\right]\left[\mathbf{c}^{\prime}\left(e^{-i \lambda}\right) \mathbf{G}_{\mathbf{y y}}^{-1}(\lambda) \mathbf{c}\left(e^{i \lambda}\right)\right] \frac{\partial G_{x x}(\lambda)}{\partial \boldsymbol{\theta}_{x}} \frac{\partial G_{x x}(\lambda)}{\partial \boldsymbol{\theta}_{x}^{\prime}} . }
\end{aligned}
$$

Further

$$
\begin{aligned}
& \frac{\partial v e c^{\prime}\left[\mathbf{G}_{\mathbf{y y}}(\lambda)\right]}{\partial \boldsymbol{\theta}_{x}}\left[\mathbf{G}_{\mathbf{y y}}^{-1}(\lambda) \otimes \mathbf{G}_{\mathbf{y y}}^{\prime-1}(\lambda)\right] \frac{\partial v e c\left[\mathbf{G}_{\mathbf{y y}}(\lambda)\right]}{\partial \boldsymbol{\theta}_{u_{i}}^{\prime}} \\
= & \frac{\partial G_{x x}(\lambda)}{\partial \boldsymbol{\theta}_{x}}\left[\mathbf{c}^{\prime}\left(e^{i \lambda}\right) \otimes \mathbf{c}^{\prime}\left(e^{-i \lambda}\right)\right]\left[\mathbf{G}_{\mathbf{y y}}^{-1}(\lambda) \otimes \mathbf{G}_{\mathbf{y y}}^{\prime-1}(\lambda)\right] \mathbf{E}_{N} \mathbf{e}_{j} \frac{\partial G_{u_{j} u_{j}}(\lambda)}{\partial \boldsymbol{\theta}_{u_{j}}^{\prime}} \\
= & {\left[\mathbf{c}^{\prime}\left(e^{i \lambda}\right) \mathbf{G}_{\mathbf{y y}}^{-1}(\lambda) \mathbf{e}_{j}\right]\left[\mathbf{c}^{\prime}\left(e^{-i \lambda}\right) \mathbf{G}_{\mathbf{y y}}^{\prime-1}(\lambda) \mathbf{e}_{j}\right] \frac{\partial G_{x x}(\lambda)}{\partial \boldsymbol{\theta}_{x}} \frac{\partial G_{u_{j} u_{j}}(\lambda)}{\partial \boldsymbol{\theta}_{u_{j}}^{\prime}} . }
\end{aligned}
$$

Finally,

$$
\begin{aligned}
& \frac{\partial v e c^{\prime}\left[\mathbf{G}_{\mathbf{y y}}(\lambda)\right]}{\partial \boldsymbol{\theta}_{u_{i}}}\left[\mathbf{G}_{\mathbf{y y}}^{-1}(\lambda) \otimes \mathbf{G}_{\mathbf{y y}}^{\prime-1}(\lambda)\right] \frac{\partial v e c\left[\mathbf{G}_{\mathbf{y y}}(\lambda)\right]}{\partial \boldsymbol{\theta}_{u_{j}}^{\prime}} \\
= & \frac{\partial G_{u_{i} u_{i}}(\lambda)}{\partial \boldsymbol{\theta}_{u_{i}}} \mathbf{e}_{i}^{\prime} \mathbf{E}_{N}^{\prime}\left[\mathbf{G}_{\mathbf{y y}}^{-1}(\lambda) \otimes \mathbf{G}_{\mathbf{y y}}^{\prime-1}(\lambda)\right] \mathbf{E}_{N} \mathbf{e}_{j} \frac{\partial G_{u_{j} u_{j}}(\lambda)}{\partial \boldsymbol{\theta}_{u_{j}}^{\prime}} \\
= & \mathbf{e}_{i}^{\prime}\left[\mathbf{G}_{\mathbf{y y}}^{-1}(\lambda) \odot \mathbf{G}_{\mathbf{y y}}^{\prime-1}(\lambda)\right] \mathbf{e}_{j} \frac{\partial G_{u_{i} u_{i}}(\lambda)}{\partial \boldsymbol{\theta}_{u_{i}}} \frac{\partial G_{u_{j} u_{j}}(\lambda)}{\partial \boldsymbol{\theta}_{u_{j}}^{\prime}},
\end{aligned}
$$

where $\odot$ denotes the Hadamard (or element by element) product of two matrices of equal size.

If we assume that both $G_{x x}(\lambda)$ and $\mathbf{G}_{\mathbf{u u}}(\lambda)$ are strictly positive, we can use again the Woodbury formula to considerably simplify the previous expressions. In particular,

$$
\begin{aligned}
\mathbf{G}_{\mathbf{y y}}^{-1}(\lambda) \mathbf{c}\left(e^{-i \lambda}\right) & =\mathbf{G}_{\mathbf{u u}}^{-1}(\lambda) \mathbf{c}\left(e^{-i \lambda}\right)-\omega(\lambda) \mathbf{G}_{\mathbf{u u}}^{-1}(\lambda) \mathbf{c}\left(e^{-i \lambda}\right) \mathbf{c}^{\prime}\left(e^{i \lambda}\right) \mathbf{G}_{\mathbf{u u}}^{-1}(\lambda) \mathbf{c}\left(e^{-i \lambda}\right), \\
\mathbf{G}_{\mathbf{y y}}^{\prime-1}(\lambda) \mathbf{c}\left(e^{i \lambda}\right) & =\mathbf{G}_{\mathbf{u u}}^{-1}(\lambda) \mathbf{c}\left(e^{i \lambda}\right)-\omega(\lambda) \mathbf{G}_{\mathbf{u u}}^{-1}(\lambda) \mathbf{c}\left(e^{i \lambda}\right) \mathbf{c}^{\prime}\left(e^{-i \lambda}\right) \mathbf{G}_{\mathbf{u u}}^{-1}(\lambda) \mathbf{c}\left(e^{i \lambda}\right),
\end{aligned}
$$

so that

$$
\mathbf{c}^{\prime}\left(e^{i \lambda}\right) \mathbf{G}_{\mathbf{y y}}^{-1}(\lambda) \mathbf{c}\left(e^{-i \lambda}\right)=\left[\mathbf{c}^{\prime}\left(e^{-i \lambda}\right) \mathbf{G}_{\mathbf{y y}}^{-1}(\lambda) \mathbf{c}\left(e^{i \lambda}\right)\right]=\mathbf{c}^{\prime}\left(e^{i \lambda}\right) \mathbf{G}_{\mathbf{u u}}^{-1}(\lambda) \mathbf{c}\left(e^{-i \lambda}\right) G_{x x}^{-1}(\lambda) \omega(\lambda)
$$

in view of (A2). Finally, further speed gains can be achieved by noticing that

$$
\mathbf{c}^{\prime}\left(e^{i \lambda}\right) \mathbf{G}_{\mathbf{u u}}^{-1}(\lambda) \mathbf{c}\left(e^{-i \lambda}\right)=\sum_{j=1}^{N} \frac{\left\|c_{j}\left(e^{i \lambda}\right)\right\|^{2}}{G_{u_{j} u_{j}}(\lambda)} .
$$




\section{F A simple example with pure AR processes}

\section{F.1 The model and spectral density}

We illustrate our spectral EM algorithm with the following model

$$
\begin{aligned}
\left(\begin{array}{c}
y_{1, t} \\
\vdots \\
y_{N, t}
\end{array}\right) & =\left(\begin{array}{c}
\mu_{1} \\
\vdots \\
\mu_{N}
\end{array}\right)+\left(\begin{array}{c}
c_{1,0} \\
\vdots \\
c_{N, 0}
\end{array}\right) x_{t}+\left(\begin{array}{c}
c_{1,1} \\
\vdots \\
c_{N, 1}
\end{array}\right) x_{t-1}+\left(\begin{array}{c}
u_{1, t} \\
\vdots \\
u_{N, t}
\end{array}\right), \\
x_{t} & =\alpha_{x 1} x_{t-1}+f_{t} \\
u_{i t} & =\alpha_{u_{i} 1} u_{i t-1}+v_{i t}, \quad i=1, \ldots, N
\end{aligned}
$$

In this case:

$$
\begin{aligned}
G_{x x}(\lambda) & =\frac{1}{1+\alpha_{x 1}^{2}-2 \alpha_{x 1} \cos \lambda} \psi_{f}, \\
G_{u_{i} u_{i}}(\lambda) & =\frac{1}{1+\alpha_{u_{i} 1}^{2}-2 \alpha_{u_{i} 1} \cos \lambda} \psi_{i},
\end{aligned}
$$

and

$$
\mathbf{c}\left(e^{-i \lambda t}\right)=\mathbf{c}_{0}+\mathbf{c}_{1} e^{-i \lambda}=\left(\begin{array}{c}
c_{1,0}+c_{1,1} e^{-i \lambda} \\
\vdots \\
c_{N, 0}+c_{N, 1} e^{-i \lambda}
\end{array}\right)=\left(\begin{array}{c}
c_{1}\left(e^{-i \lambda t}\right) \\
\vdots \\
c_{N}\left(e^{-i \lambda t}\right)
\end{array}\right)
$$

\section{F.2 Complete likelihood}

Using the results in section 4 , the derivative of $G_{x x}(\lambda)$ with respect to $\alpha_{x 1}$ would be

$$
\frac{\partial G_{x x}(\lambda)}{\partial \alpha_{x 1}}=\frac{2\left(\cos \lambda-\alpha_{x 1}\right)}{\left(1+\alpha_{x 1}^{2}-2 \alpha_{x 1} \cos \lambda\right)^{2}} .
$$

Hence, the log-likelihood score would become

$$
\begin{aligned}
\frac{\partial l(\mathbf{x})}{\partial \alpha_{x 1}} & =\frac{1}{2} \sum_{j=0}^{T-1} \frac{2\left(\cos \lambda_{j}-\alpha_{x 1}\right)}{\left(1+\alpha_{x 1}^{2}-2 \alpha_{x 1} \cos \lambda_{j}\right)^{2}}\left(1+\alpha_{x 1}^{2}-2 \alpha_{x 1} \cos \lambda_{j}\right)^{2} \times \\
& \times\left[2 \pi z_{j}^{x} z_{j}^{x *}-\frac{1}{\left(1+\alpha_{x 1}^{2}-2 \alpha_{x 1} \cos \lambda_{j}\right)}\right]=2 \pi \sum_{j=0}^{T-1}\left(\cos \lambda_{j}-\alpha_{x 1}\right) z_{j}^{x} z_{j}^{x *}
\end{aligned}
$$

where we have exploited the fact that

$$
\sum_{j=0}^{T-1} \frac{\left(\cos \lambda_{j}-\alpha_{x 1}\right)}{\left(1+\alpha_{x 1}^{2}-2 \alpha_{x 1} \cos \lambda_{j}\right)}=\gamma_{x x}(1)-\alpha_{x 1} \gamma_{x x}(0)=0
$$

As a result, if we set the score to 0 and solve for $\alpha_{x 1}$ then we would obtain

$$
\hat{\alpha}_{x 1}=\frac{\sum_{j=0}^{T-1} \cos \lambda_{j} z_{j}^{x} z_{j}^{x *}}{\sum_{j=0}^{T-1} z_{j}^{x} z_{j}^{x *}}=\frac{\sum_{j=0}^{T-1} \cos \lambda_{j} I_{x x}\left(\lambda_{j}\right)}{\sum_{j=0}^{T-1} I_{x x}\left(\lambda_{j}\right)} .
$$

But since

$$
I_{x x}\left(\lambda_{j}\right)=\hat{\gamma}_{x x}(0)+2 \sum_{k=1}^{T-1} \hat{\gamma}_{x x}(k) \cos \left(k \lambda_{j}\right)
$$


we would have that

$$
\sum_{j=0}^{T-1} 2 \pi I_{x x}\left(\lambda_{j}\right)=T \hat{\gamma}_{x x}(0)
$$

and

$$
\sum_{j=0}^{T-1} \cos \lambda_{j}\left[2 \pi I_{x x}\left(\lambda_{j}\right)\right]=T\left[\hat{\gamma}_{x x}(1)+\hat{\gamma}_{x x}(T-1)\right],
$$

which is the first sample (circulant) autocovariance of $x_{t}$. Therefore, the expression for $\hat{\alpha}_{x 1}$ is (almost) identical to the one we would obtain in the time domain, which will be given by $\hat{\gamma}_{x x}(1) / \hat{\gamma}_{x x}(0)$, because $\hat{\gamma}_{x x}(T-1)=T^{-1} x_{T} x_{1}=o_{p}(1)$.

Similar expressions would apply to the dynamic parameters that appear in $\boldsymbol{\theta}_{u_{i}}$ for a given value of $\mathbf{c}_{i}$. in view of (11b), since in this case it would be possible to estimate the variances of the innovations $\psi_{i}$ in closed form.

Specifically, the partial derivatives of $G_{u_{i} u_{i}}(\lambda)$ with respect to $\psi_{i}$ and $\alpha_{u_{i} 1}$ would be

$$
\begin{aligned}
\frac{\partial G_{u_{i} u_{i}}(\lambda)}{\partial \psi_{i}} & =\frac{1}{1+\alpha_{u_{i} 1}^{2}-2 \alpha_{u_{i} 1} \cos \lambda}, \\
\frac{\partial G_{u_{i} u_{i}}(\lambda)}{\partial \alpha_{u_{i} 1}} & =\frac{2\left(\cos \lambda-\alpha_{u_{i} 1}\right) \psi_{i}}{\left(1+\alpha_{u_{i} 1}^{2}-2 \alpha_{u_{i} 1} \cos \lambda\right)^{2}}
\end{aligned}
$$

so the corresponding log-likelihood scores would be

$$
\begin{aligned}
\frac{\partial l\left(\mathbf{y}_{i} \mid \mathbf{x}\right)}{\partial \psi_{i}} & =\frac{1}{2} \sum_{j=0}^{T-1} \frac{\left(1+\alpha_{u_{i} 1}^{2}-2 \alpha_{u_{i} 1} \cos \lambda_{j}\right)^{2}}{\left(1+\alpha_{u_{i} 1}^{2}-2 \alpha_{u_{i} 1} \cos \lambda_{j}\right) \psi_{i}^{2}}\left[2 \pi z_{j}^{u_{i}} z_{j}^{u_{i} *}-\frac{\psi_{i}}{1+\alpha_{u_{i} 1}^{2}-2 \alpha_{u_{i} 1} \cos \lambda_{j}}\right] \\
& =\frac{1}{2 \psi_{i}^{2}} \sum_{j=0}^{T-1}\left[\left(1+\alpha_{u_{i} 1}^{2}-2 \alpha_{u_{i} 1} \cos \lambda_{j}\right) 2 \pi z_{j}^{u_{i}} z_{j}^{u_{i} *}-\psi_{i}\right] \\
\frac{\partial l\left(\mathbf{y}_{i} \mid \mathbf{x}\right)}{\partial \alpha_{u_{i} 1}} & =\frac{1}{2} \sum_{j=0}^{T-1} \frac{2\left(\cos \lambda_{j}-\alpha_{u_{i} 1}\right) \psi_{i}\left(1+\alpha_{u_{i} 1}^{2}-2 \alpha_{u_{i} 1} \cos \lambda_{j}\right)^{2}}{\left(1+\alpha_{u_{i} 1}^{2}-2 \alpha_{u_{i} 1} \cos \lambda_{j}\right)^{2} \psi_{i}^{2}} \\
& \times\left[2 \pi z_{j}^{u_{i}} z_{j}^{u_{i} *}-\frac{\psi_{i}}{\left(1+\alpha_{x 1}^{2}-2 \alpha_{x 1} \cos \lambda_{j}\right)}\right]=\frac{2 \pi}{\psi_{i}} \sum_{j=0}^{T-1}\left(\cos \lambda_{j}-\alpha_{u_{i} 1}\right) z_{j}^{u_{i}} z_{j}^{u_{i} *} .
\end{aligned}
$$

Hence, the spectral ML estimators of $\psi_{i}$ and $\alpha_{u_{i} 1}$ for fixed values of $\mathbf{c}_{i}$. would satisfy

$$
\begin{aligned}
\tilde{\psi}_{i} & =\frac{2 \pi}{T} \sum_{j=0}^{T-1}\left(1+\tilde{\alpha}_{u_{i} 1}^{2}-2 \tilde{\alpha}_{u_{i} 1} \cos \lambda_{j}\right) z_{j}^{u_{i}} z_{j}^{u_{i} *}, \\
\tilde{\alpha}_{u_{i} 1} & =\frac{\sum_{j=0}^{T-1} \cos \lambda_{j} z_{j}^{u_{i}} z_{j}^{u_{i} *}}{\sum_{j=0}^{T-1} z_{j}^{u_{i}} z_{j}^{u_{i} *}} .
\end{aligned}
$$

Intuitively, these parameter estimates are, respectively, the sample analogues to the variance of $v_{i t}$, which is the residual variance in the regression of $u_{i t}$ on $u_{i t-1}$, and the slope coefficient in the same regression. 
Finally, as far as the dynamic loading parameters are concerned, the matrix on the left hand of the normal equations becomes

$$
\begin{aligned}
& \sum_{j=0}^{T-1} G_{u_{i} u_{i}}^{-1}\left(\lambda_{j}\right)\left(\begin{array}{cc}
2 z_{j}^{x} z_{j}^{x *} & \left(e^{-i \lambda_{j}}+e^{i \lambda_{j}}\right) z_{j}^{x} z_{j}^{x *} \\
\left(e^{i \lambda_{j}}+e^{-i \lambda_{j}}\right) z_{j}^{x} z_{j}^{x *} & 2 z_{j}^{x} z_{j}^{x *}
\end{array}\right) \\
= & \sum_{j=0}^{T-1} G_{u_{i} u_{i}}^{-1}\left(\lambda_{j}\right) 2 z_{j}^{x} z_{j}^{x *}\left(\begin{array}{cc}
1 & \cos \lambda_{j} \\
\cos \lambda_{j} & 1
\end{array}\right),
\end{aligned}
$$

while the vector on the right hand side will be

$$
\sum_{j=0}^{T-1} G_{u_{i} u_{i}}^{-1}\left(\lambda_{j}\right)\left(\begin{array}{c}
z_{j}^{y_{i}} z_{j}^{x *}+z_{j}^{y_{i} *} z_{j}^{x} \\
e^{i \lambda_{j}} z_{j}^{y_{j}} z_{j}^{x *}+e^{-i \lambda_{j}} z_{j}^{y_{i} *} z_{j}^{x}
\end{array}\right) .
$$

\section{F.3 Expected log-likelihood}

The expected log-likelihood score for the autoregressive parameter of the common factor becomes

$$
\frac{\partial E\left[l(\mathbf{x}) \mid \mathbf{Y}, \boldsymbol{\theta}^{(n)}\right]}{\partial \alpha_{x 1}}=2 \pi \sum_{j=0}^{T-1}\left(\cos \lambda_{j}-\alpha_{x 1}\right)\left[I_{x^{K} x^{K}}^{(n)}\left(\lambda_{j}\right)+\omega^{(n)}\left(\lambda_{j}\right)\right] .
$$

Hence,

$$
\hat{\alpha}_{x 1}^{(n+1)}=\frac{\sum_{j=0}^{T-1} \cos \lambda_{j}\left[I_{x^{K} x^{K}}^{(n)}\left(\lambda_{j}\right)+\omega^{(n)}\left(\lambda_{j}\right)\right]}{\sum_{j=0}^{T-1}\left[I_{x^{K} x^{K}}^{(n)}\left(\lambda_{j}\right)+\omega^{(n)}\left(\lambda_{j}\right)\right]} .
$$

Similarly,

$$
\begin{aligned}
\frac{\partial E\left[l\left(\mathbf{y}_{i} \mid \mathbf{x}\right) \mid \mathbf{Y}, \boldsymbol{\theta}^{(n)}\right]}{\partial \psi_{i}} & =\frac{1}{2 \psi_{i}^{2}} \sum_{j=0}^{T-1}\left(1+\alpha_{u_{i} 1}^{2}-2 \alpha_{u_{i} 1} \cos \lambda\right)\left[2 \pi I_{u_{i} u_{i}}^{(n)}\left(\lambda_{j}\right)-\psi_{i}\right] \\
\frac{E\left[l\left(\mathbf{y}_{i} \mid \mathbf{x}\right) \mid \mathbf{Y}, \boldsymbol{\theta}^{(n)}\right]}{\partial \alpha_{u_{i} 1}} & =\frac{2 \pi}{\psi_{i}} \sum_{j=0}^{T-1}\left(\cos \lambda_{j}-\alpha_{u_{i} 1}\right) I_{u_{i} u_{i}}^{(n)}\left(\lambda_{j}\right) .
\end{aligned}
$$

As a result, the spectral ML estimators of $\psi_{i}$ and $\alpha_{u_{i} 1}$ given $\mathbf{c}_{i}$. will satisfy

$$
\begin{aligned}
\hat{\psi}_{i}^{(n+1)} & =\frac{2 \pi}{T} \sum_{j=0}^{T-1}\left[1+\left(\hat{\alpha}_{u_{i} 1}^{(n+1)}\right)^{2}-2 \hat{\alpha}_{u_{i} 1}^{(n+1)} \cos \lambda_{j}\right] I_{u_{i} u_{i}}^{(n)}\left(\lambda_{j}\right), \\
\hat{\alpha}_{u_{i} 1}^{(n+1)} & =\frac{\sum_{j=0}^{T-1} \cos \lambda_{j} I_{u_{i} u_{i}}^{(n)}\left(\lambda_{j}\right)}{\sum_{j=0}^{T-1} I_{u_{i} u_{i}}^{(n)}\left(\lambda_{j}\right)} .
\end{aligned}
$$

Finally, the matrix on the left hand of the normal equations for the example in (F9) becomes

$$
\sum_{j=0}^{T-1} G_{u_{i} u_{i}}^{-1}\left(\lambda_{j}\right) 2\left[I_{x^{K} x^{K}}^{(n)}\left(\lambda_{j}\right)+\omega^{(n)}\left(\lambda_{j}\right)\right]\left(\begin{array}{cc}
1 & \cos \lambda_{j} \\
\cos \lambda_{j} & 1
\end{array}\right),
$$

while the vector on the right hand side will be

$$
\sum_{j=0}^{T-1} G_{u_{i} u_{i}}^{-1}\left(\lambda_{j}\right)\left(\begin{array}{c}
I_{y_{i} x^{K}}^{(n)}\left(\lambda_{j}\right)+I_{x^{K} y_{i}}^{(n)}\left(\lambda_{j}\right) \\
e^{i \lambda_{j}} I_{y_{i} x^{K}}^{(n)}\left(\lambda_{j}\right)+e^{-i \lambda_{j}} I_{x^{K} y_{i}}^{(n)}\left(\lambda_{j}\right)
\end{array}\right) .
$$




\section{G State space representation in the time domain}

There are several ways of casting the dynamic factor model in (F8) into state-space format, but the most straightforward one is to consider a huge state vector of dimension $2 N+3$ in which the $\operatorname{Arma}(1,1)$ process for the common factor is written as a trivariate $\operatorname{VAR}(1)$ in $\left(x_{t}, x_{t-1}, f_{t}\right)$ and the $N$ ARMA $(1,1)$ processes for the specific factors are written as first order bivariate VARs in $\left(u_{i t}, v_{i t}\right)$. As a result, we can write the measurement equation without an error term as

$$
\begin{gathered}
\mathbf{y}_{t}=\mathbf{\Gamma} \mathbf{x}_{t} \\
\mathbf{x}_{t}=\left(x_{t}, x_{t-1}, f_{t} ; u_{1 t}, v_{1 t} ; \ldots ; u_{i t}, v_{i t} ; \ldots ; u_{N t}, v_{N t}\right)^{\prime}
\end{gathered}
$$

and $\boldsymbol{\Gamma}$ is an $N \times(2 N+3)$ matrix with typical row equal to

$$
\left[c_{i 0}, c_{i 1}, 0 ; 0,0 ; \ldots ; 1,0 ; \ldots ; 0,0\right]
$$

In turn, the transition equation will be

$$
\begin{aligned}
{\left[\begin{array}{c}
x_{t} \\
x_{t-1} \\
f_{t}
\end{array}\right] } & =\left[\begin{array}{ccc}
\alpha & 0 & -\beta \\
1 & 0 & 0 \\
0 & 0 & 0
\end{array}\right]\left[\begin{array}{l}
x_{t-1} \\
x_{t-2} \\
f_{t-1}
\end{array}\right]+\left[\begin{array}{c}
f_{t} \\
0 \\
f_{t}
\end{array}\right], \\
{\left[\begin{array}{c}
u_{i t} \\
v_{i t}
\end{array}\right] } & =\left[\begin{array}{cc}
\alpha & -\beta \\
0 & 0
\end{array}\right]\left[\begin{array}{l}
u_{i t-1} \\
v_{i t-1}
\end{array}\right]+\left[\begin{array}{l}
v_{i t} \\
v_{i t}
\end{array}\right] \quad(i=1, \ldots, N),
\end{aligned}
$$

with a block diagonal covariance matrix for its innovations.

Given our stationary assumption, the initial conditions for the state will trivially be $\mathbf{x}_{1 \mid 0}=$ $\mathbf{0}_{(2 N+3) x 1}$ and

$$
\mathbf{P}_{1 \mid 0}=\left[\begin{array}{cccccc}
\mathbf{Q}_{x} & \mathbf{0} & \cdots & \cdots & \cdots & \mathbf{0} \\
\mathbf{0} & \mathbf{Q}_{1} & \cdots & \cdots & \cdots & \mathbf{0} \\
\vdots & \vdots & \ddots & \ddots & \ddots & \vdots \\
\vdots & \vdots & \ddots & \mathbf{Q}_{i} & \ddots & \vdots \\
\vdots & \vdots & \ddots & \ddots & \ddots & \mathbf{0} \\
\mathbf{0} & \mathbf{0} & \cdots & \cdots & \mathbf{0} & \mathbf{Q}_{N}
\end{array}\right]
$$

in which the first $3 \times 3$ block is

$$
\mathbf{Q}_{x}=\left[\begin{array}{ccc}
\gamma_{x 0} & \gamma_{x 1} & 1 \\
\gamma_{x 1} & \gamma_{x 0} & 0 \\
1 & 0 & 1
\end{array}\right], \quad \gamma_{x 0}=\frac{1+\beta^{2}-2 \alpha \beta}{1-\alpha^{2}}, \gamma_{x 1}=\frac{(1-\alpha \beta)(\alpha-\beta)}{1-\alpha^{2}}
$$

and the other $N 2 \times 2$ blocks are

$$
\mathbf{Q}_{i}=\left[\begin{array}{cc}
\gamma_{i 0} & \psi_{i} \\
\psi_{i} & \psi_{i}
\end{array}\right], \quad \gamma_{i 0}=\frac{1+\beta_{i}^{2}-2 \alpha_{i} \beta_{i}}{1-\alpha_{i}^{2}} \psi_{i} .
$$



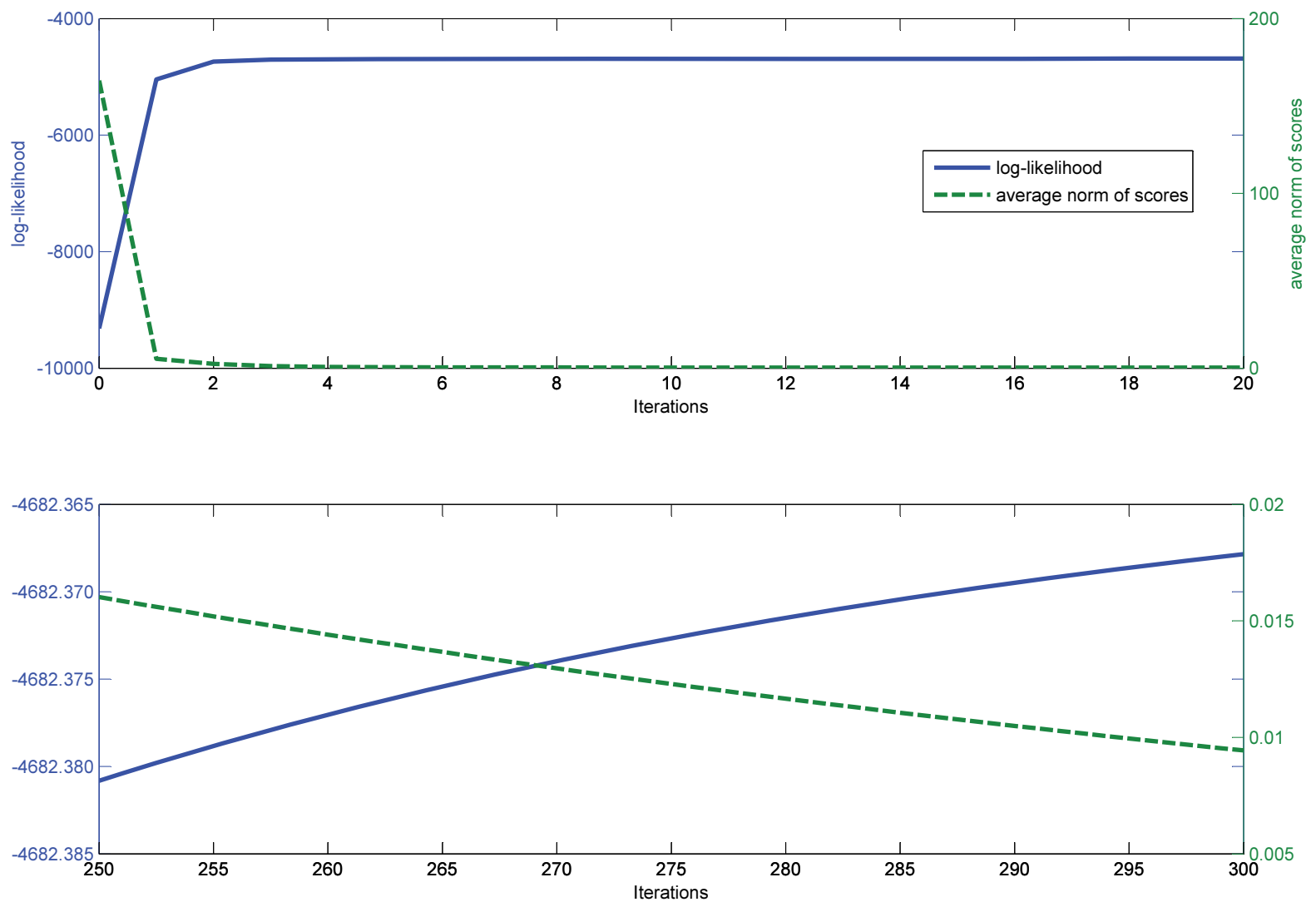

Figure 1: A model with $N=10$ series 

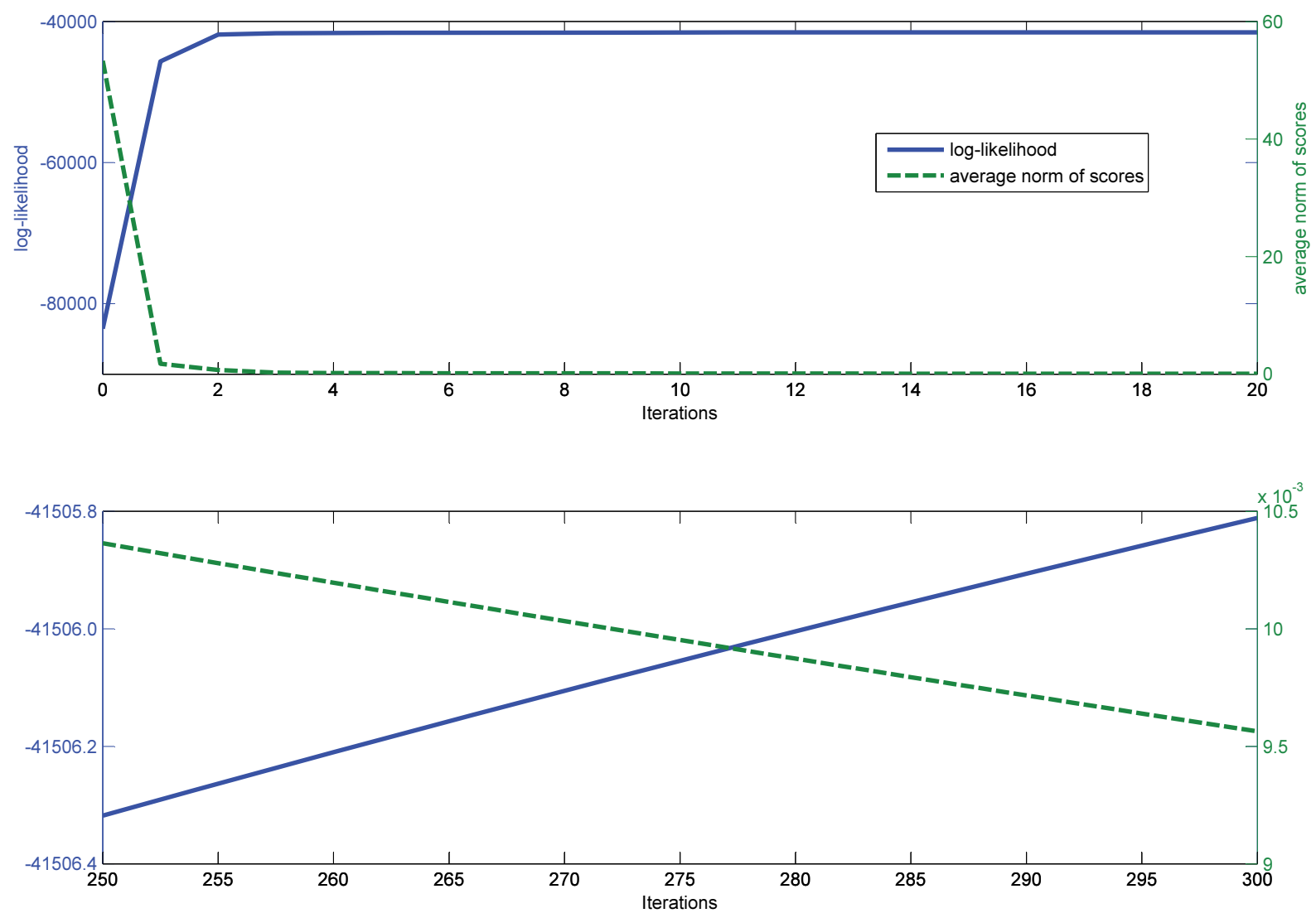

Figure 2: A model with $N=100$ series 


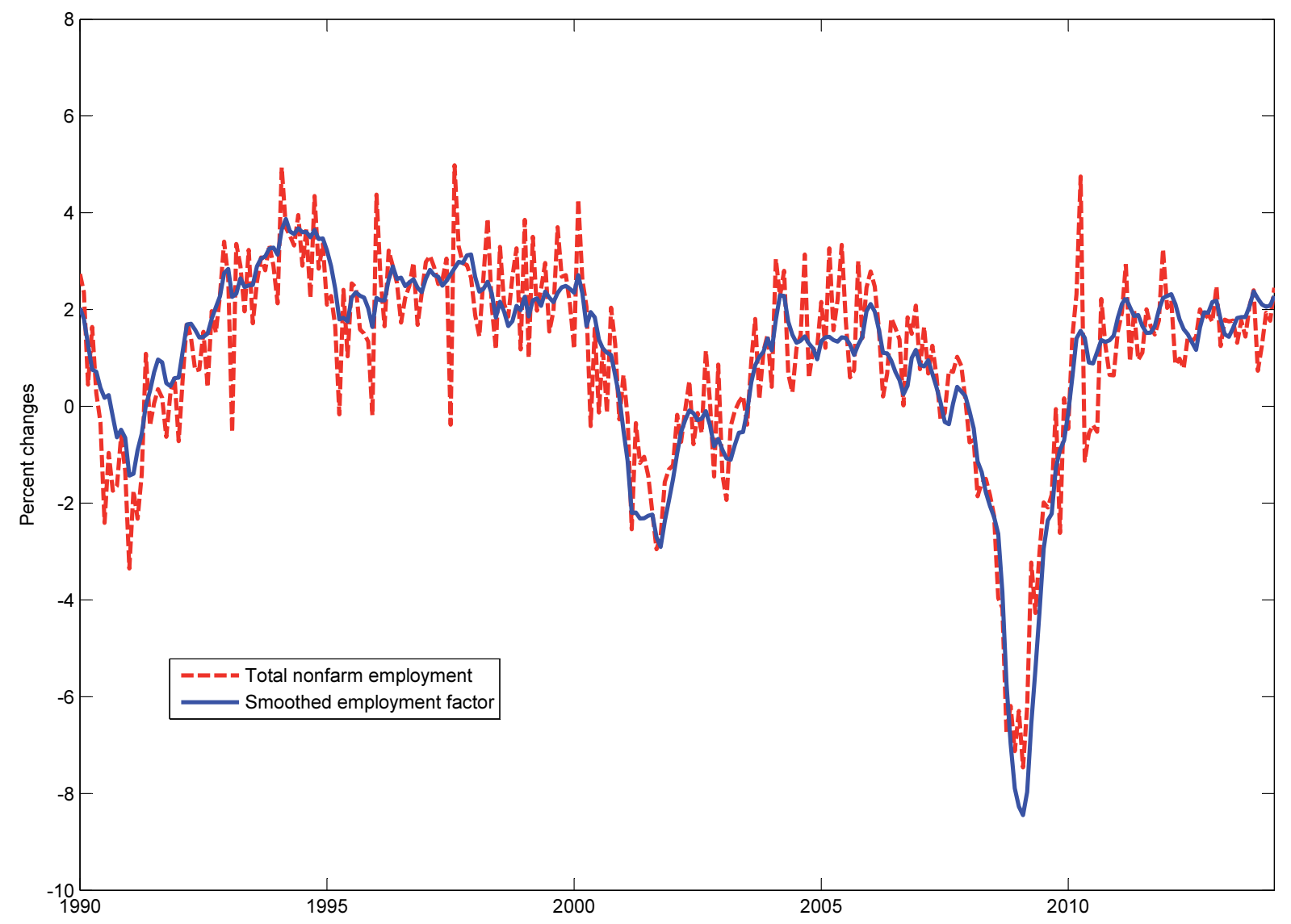

Figure 3: Total nonfarm employment and smoothed employment factor (annualised monthly frequency) 


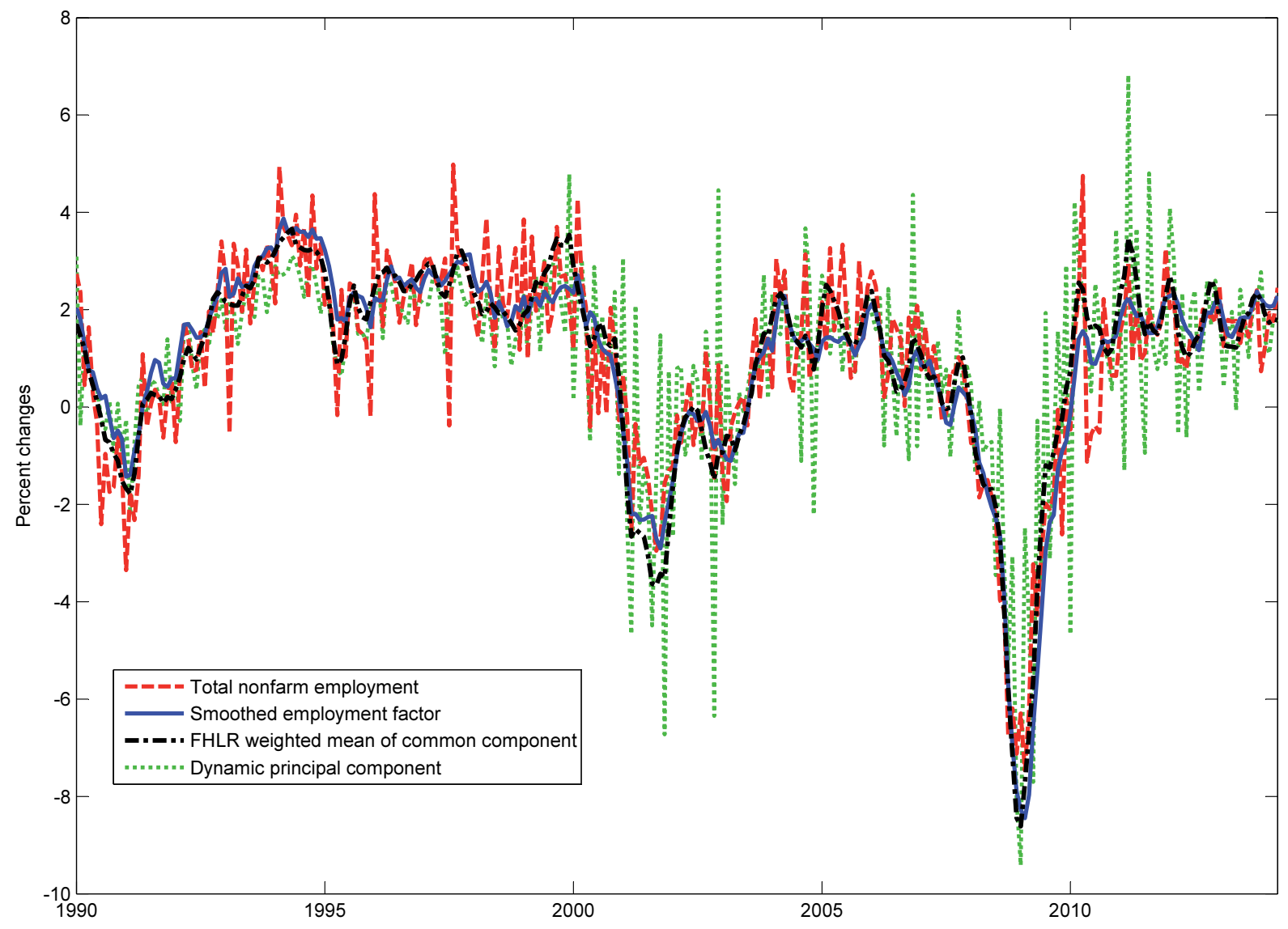

Figure 4: Total nonfarm employment and non-parametric factor estimators (annualised monthly frequency) 
Table 1: Performance of the Indirect Inference procedure for univariate $\operatorname{ArmA}(1,1)$

\begin{tabular}{llllll} 
& \multicolumn{1}{c}{$\alpha$} & $\beta$ & & \multicolumn{1}{c}{$\sigma_{f}^{2}$} & Relative time \\
\hline \hline True values & 0.95 & -0.50 & 1.00 & \\
GMM & $0.9428(0.0291)$ & -0.4457 & $(0.1461)$ & $1.1200(0.2496)$ & 0.0029 \\
Ind. Inf. (1 iteration) & $0.9431(0.0287)$ & -0.4453 & $(0.1169)$ & $1.1087(0.2509)$ & 0.0080 \\
Ind. Inf. (3 iterations) & $0.9429(0.0287)$ & $-0.4473(0.1117)$ & $1.1076(0.2477)$ & 0.0208 \\
Ind. Inf. (5 iterations) & $0.9432(0.0286)$ & -0.4472 & $(0.1116)$ & $1.1063(0.2463)$ & 0.0338 \\
Iterated Ind. Inf. & $0.9432(0.0286)$ & -0.4472 & $(0.1115)$ & $1.1063(0.2463)$ & 0.0375 \\
Direct ML (Quasi-Newton) & $0.9432(0.0286)$ & -0.4472 & $(0.1115)$ & $1.1063(0.2463)$ & 1 \\
& & & & & 1.00 \\
True values & 0.95 & 0.50 & & & \\
GMM & $0.9428(0.0430)$ & 0.4805 & $(0.1532)$ & $1.0198(0.1201)$ & 0.0028 \\
Ind. Inf. (1 iteration) & $0.9420(0.0344)$ & 0.4800 & $(0.0856)$ & $1.0093(0.1133)$ & 0.0073 \\
Ind. Inf. (3 iterations) & $0.9420(0.0335)$ & 0.4814 & $(0.0816)$ & $1.0079(0.1137)$ & 0.0190 \\
Ind. Inf. (5 iterations) & $0.9420(0.0334)$ & 0.4816 & $(0.0818)$ & $1.0079(0.1139)$ & 0.0307 \\
Iterated Ind. Inf. & $0.9420(0.0334)$ & 0.4817 & $(0.0818)$ & $1.0074(0.1135)$ & 0.0384 \\
Direct ML (Quasi-Newton) & $0.9420(0.0334)$ & 0.4817 & $(0.0818)$ & $1.0074(0.1135)$ & 1 \\
& & & & & \\
\hline True values & 0.95 & 0.70 & & & \\
GMM & $0.9079(0.0936)$ & 0.6633 & $(0.2522)$ & $1.0488(0.1928)$ & 0.0023 \\
Ind. Inf. (1 iteration) & $0.9475(0.0484)$ & 0.6941 & $(0.1230)$ & $1.0217(0.1501)$ & 0.0068 \\
Ind. Inf. (3 iterations) & $0.9464(0.0456)$ & 0.6924 & $(0.1027)$ & $1.0126(0.1294)$ & 0.0172 \\
Ind. Inf. (5 iterations) & $0.9445(0.0421)$ & 0.6892 & $(0.0906)$ & $1.0036(0.1156)$ & 0.0288 \\
Iterated Ind. Inf. & $0.9432(0.0402)$ & 0.6853 & $(0.0805)$ & $0.9993(0.1099)$ & 0.0972 \\
Direct ML (Quasi-Newton) & $0.9424(0.0396)$ & 0.6837 & $(0.0786)$ & $0.9970(0.1075)$ & 1 \\
\hline
\end{tabular}

Monte Carlo medians and (interquartile ranges) of estimated parameters and computation time relative to direct maximization of the log-likelihood. Model: $(1-\alpha L) x_{t}=(1-\beta L) f_{t}$. Sample length=300. Replications $=2,000$. 
Table 2: Convergence properties of the EM algorithm

$\%$ maximum distance covered Log-likelihood. Relative time.

\begin{tabular}{lccc}
\hline \hline Initial values & 0 & -11519.2457 & 0 \\
5 EM iterations & 70.36 & -4695.3231 & 0.0158 \\
10 EM iterations & 76.63 & -4687.7697 & 0.0317 \\
300 EM iterations & 98.83 & -4669.6352 & 0.9503 \\
5 EM iterations + QN & 100 & -4669.5494 & 0.5341 \\
10 EM iterations + QN & 100 & -4669.5494 & 0.5174 \\
QN & 100 & -4669.5494 & 1 \\
\hline \hline
\end{tabular}

Monte Carlo averages of the percentage of the maximum distance covered, of the log-likelihood values and of the computation time relative to Quasi-Newton. The maximum distance is assumed to be the $L^{2}$ norm of the difference between the parameter initial values and the QN estimates. Sample length $=300$. Replications $=100$. 
Table 3: Sample of NAICS 3-digits sectors for estimating the employment index

Oil and gas extraction (211)

Mining, except oil and gas (212)

Support activities for mining (213)

Construction of buildings (236)

Heavy and civil engineering construction (237)

Specialty trade contractors (238)

Wood products (321)

Nonmetallic mineral products (327)

Primary metals (331)

Fabricated metal products (332)

Machinery (333)

Computer and electronic products (334)

Electrical equipment and appliances (335)

Transportation equipment (336)

Furniture and related products (337)

Miscellaneous durable goods manufacturing (339)

Food manufacturing (311)

Textile mills (313)

Textile product mills (314)

Apparel (315)

Paper and paper products (322)

Printing and related support activities (323)

Petroleum and coal products (324)

Chemicals (325)

Plastics and rubber products (326)

Miscellaneous nondurable goods manufacturing $(312,6)$

Wholesale trade, durable goods (423)

Wholesale trade, nondurable goods (424)

Electronic markets and agents and brokers (425)

Motor vehicle and parts dealers (441)

Furniture and home furnishings stores (442)

Electronics and appliance stores (443)

Building material and garden supply stores (444)

Food and beverage stores (445)

Health and personal care stores (446)

Gasoline stations (447)

Clothing and clothing accessories stores (448)

Sporting goods, hobby, book, and music stores (451)

General merchandise stores (452)

Miscellaneous store retailers (453)

Nonstore retailers (454)

Air transportation (481)

Rail transportation (482)

Water transportation (483)

Truck transportation (484)

Transit and ground passenger transportation (485)

Pipeline transportation (486)

Scenic and sightseeing transportation (487)

Support activities for transportation (488)

Couriers and messengers (492)
Warehousing and storage (493)

Publishing industries, except Internet (511)

Motion picture and sound recording industries (512)

Broadcasting, except Internet (515)

Telecommunications (517)

Data processing, hosting and related services (518)

Other information services (519)

Monetary authorities - central bank (521)

Credit intermediation and related activities (522)

Securities, commodity contracts, investments, etc. $(523,5)$

Insurance carriers and related activities (524)

Real estate (531)

Rental and leasing services (532)

Lessors of nonfinancial intangible assets (533)

Administrative and support services (561)

Waste management and remediation services (562)

Ambulatory health care services (621)

Hospitals (622)

Nursing and residential care facilities (623)

Social assistance (624)

Performing arts and spectator sports (711)

Museums, historical sites, and similar institutions (712)

Amusements, gambling, and recreation (713)

Accommodation (721)

Food services and drinking places (722)

Repair and maintenance (811)

Personal and laundry services (812)

Membership associations and organizations (813)

Federal, except U.S. Postal Service

State government, excluding education

Local government, excluding education

Notes: NAICS 3-digit codes in parentheses. 
Table 4: Dynamic loadings estimates

\begin{tabular}{|c|c|c|c|c|c|c|c|c|c|}
\hline Series & $c_{i, 0}$ & std.err. & $c_{i, 1}$ & std.err. & Series & $c_{i, 0}$ & std.err. & $c_{i, 1}$ & std.err. \\
\hline 1 & 0.510 & $(0.458)$ & -0.183 & $(0.458)$ & 42 & 0.956 & $(0.401)$ & -0.469 & $(0.400)$ \\
\hline 2 & 0.606 & $(0.542)$ & -0.201 & $(0.542)$ & 43 & 0.026 & $(0.394)$ & 0.275 & $(0.395)$ \\
\hline 3 & 0.233 & $(0.657)$ & 1.180 & $(0.663)$ & 44 & 0.049 & $(0.748)$ & 0.178 & $(0.748)$ \\
\hline 4 & 1.757 & $(0.335)$ & -0.957 & $(0.331)$ & 45 & 1.080 & $(0.235)$ & -0.522 & $(0.232)$ \\
\hline 5 & 2.004 & $(0.499)$ & -1.343 & $(0.494)$ & 46 & 0.279 & $(0.608)$ & -0.136 & $(0.608)$ \\
\hline 6 & 2.195 & $(0.316)$ & -1.351 & $(0.308)$ & 47 & -0.520 & $(0.461)$ & 0.444 & $(0.461)$ \\
\hline 7 & 2.457 & $(0.385)$ & -1.445 & $(0.377)$ & 48 & -0.341 & $(1.523)$ & 0.836 & $(1.524)$ \\
\hline 8 & 2.031 & $(0.297)$ & -1.226 & $(0.288)$ & 49 & 0.572 & $(0.299)$ & 0.011 & $(0.300)$ \\
\hline 9 & 1.582 & $(0.295)$ & -0.135 & $(0.300)$ & 50 & 0.312 & $(0.663)$ & 0.241 & $(0.665)$ \\
\hline 10 & 1.060 & $(0.141)$ & 0.038 & $(0.149)$ & 51 & 0.877 & $(0.266)$ & -0.486 & $(0.264)$ \\
\hline 11 & 0.720 & $(0.183)$ & 0.479 & $(0.195)$ & 52 & 0.290 & $(0.135)$ & 0.247 & $(0.140)$ \\
\hline 12 & 0.447 & $(0.169)$ & 0.236 & $(0.172)$ & 53 & 1.518 & $(0.908)$ & -1.227 & $(0.906)$ \\
\hline 13 & 0.741 & $(0.226)$ & 0.166 & $(0.232)$ & 54 & -0.245 & $(0.216)$ & 0.654 & $(0.221)$ \\
\hline 14 & 1.839 & $(0.483)$ & -1.042 & $(0.478)$ & 55 & 0.136 & $(0.232)$ & 0.163 & $(0.233)$ \\
\hline 15 & 2.068 & $(0.243)$ & -1.060 & $(0.234)$ & 56 & 0.822 & $(0.339)$ & -0.617 & $(0.338)$ \\
\hline 16 & 0.625 & $(0.168)$ & -0.224 & $(0.167)$ & 57 & 0.622 & $(0.430)$ & 0.067 & $(0.430)$ \\
\hline 17 & 0.159 & $(0.200)$ & -0.069 & $(0.200)$ & 58 & -0.018 & $(0.480)$ & 0.106 & $(0.480)$ \\
\hline 18 & 2.786 & $(0.448)$ & -1.992 & $(0.437)$ & 59 & 0.030 & $(0.139)$ & 0.010 & $(0.139)$ \\
\hline 19 & 2.037 & $(0.412)$ & -1.298 & $(0.406)$ & 60 & 0.212 & $(0.220)$ & 0.337 & $(0.223)$ \\
\hline 20 & 1.760 & $(0.462)$ & -1.068 & $(0.458)$ & 61 & 0.029 & $(0.109)$ & 0.040 & $(0.109)$ \\
\hline 21 & 0.677 & $(0.154)$ & -0.289 & $(0.153)$ & 62 & 0.416 & $(0.193)$ & -0.174 & $(0.192)$ \\
\hline 22 & 0.391 & $(0.180)$ & 0.212 & $(0.185)$ & 63 & 0.456 & $(0.268)$ & 0.212 & $(0.272)$ \\
\hline 23 & 0.329 & $(0.451)$ & -0.316 & $(0.451)$ & 64 & -0.610 & $(0.745)$ & 0.847 & $(0.746)$ \\
\hline 24 & 0.168 & $(0.132)$ & 0.067 & $(0.133)$ & 65 & 2.734 & $(0.272)$ & -1.932 & $(0.255)$ \\
\hline 25 & 1.542 & $(0.236)$ & -0.694 & $(0.232)$ & 66 & 0.261 & $(0.323)$ & -0.031 & $(0.323)$ \\
\hline 26 & 0.568 & $(0.358)$ & -0.319 & $(0.357)$ & 67 & 0.260 & $(0.085)$ & -0.267 & $(0.085)$ \\
\hline 27 & 0.614 & $(0.098)$ & -0.169 & $(0.098)$ & 68 & 0.073 & $(0.063)$ & -0.051 & $(0.063)$ \\
\hline 28 & 0.593 & $(0.125)$ & -0.367 & $(0.123)$ & 69 & -0.092 & $(0.090)$ & 0.038 & $(0.090)$ \\
\hline 29 & 0.778 & $(0.156)$ & -0.502 & $(0.154)$ & 70 & -0.195 & $(0.301)$ & 0.273 & $(0.301)$ \\
\hline 30 & 1.187 & $(0.136)$ & -0.819 & $(0.132)$ & 71 & -1.157 & $(0.970)$ & 1.437 & $(0.971)$ \\
\hline 31 & 2.035 & $(0.261)$ & -1.352 & $(0.252)$ & 72 & 0.093 & $(0.429)$ & 0.174 & $(0.429)$ \\
\hline 32 & 1.547 & $(0.437)$ & -0.925 & $(0.434)$ & 73 & 0.736 & $(0.496)$ & -0.374 & $(0.495)$ \\
\hline 33 & 1.668 & $(0.275)$ & -1.202 & $(0.269)$ & 74 & 0.953 & $(0.262)$ & -0.544 & $(0.261)$ \\
\hline 34 & 0.119 & $(0.136)$ & 0.031 & $(0.137)$ & 75 & 0.683 & $(0.162)$ & -0.479 & $(0.160)$ \\
\hline 35 & 0.171 & $(0.194)$ & -0.006 & $(0.194)$ & 76 & 0.967 & $(0.204)$ & -0.587 & $(0.202)$ \\
\hline 36 & 0.205 & $(0.187)$ & -0.016 & $(0.187)$ & 77 & 0.520 & $(0.161)$ & -0.310 & $(0.160)$ \\
\hline 37 & 1.621 & $(0.365)$ & -1.326 & $(0.362)$ & 78 & 0.182 & $(0.149)$ & -0.134 & $(0.149)$ \\
\hline 38 & 1.238 & $(0.565)$ & -0.821 & $(0.563)$ & 79 & -0.717 & $(1.330)$ & 0.442 & $(1.329)$ \\
\hline 39 & 0.512 & $(0.318)$ & -0.326 & $(0.318)$ & 80 & -0.156 & $(0.113)$ & 0.203 & $(0.113)$ \\
\hline 40 & 0.828 & $(0.277)$ & -0.303 & $(0.276)$ & 81 & -0.155 & $(0.137)$ & 0.195 & $(0.137)$ \\
\hline 41 & 0.581 & $(0.420)$ & -0.128 & $(0.420)$ & & & & & \\
\hline
\end{tabular}


Table 5: ARMA parameter estimates

\begin{tabular}{|c|c|c|c|c|c|c|c|c|}
\hline ries & std.err. & $\beta$ std.err. & $\psi$ st & ries & $\alpha$ & $\beta$ & $\psi$ & std.err. \\
\hline$x$ & $0.969(0.015)$ & $-0.448(0.0$ & 1.000 & & & & & \\
\hline 1 & $0.974(0.017)$ & $828(0.0$ & $60.096(4.986)$ & 42 & $0.695(0.092)$ & $0.347(0.120)$ & ) 40.336 & $(3.358)$ \\
\hline 2 & 0.722 & 0.528 & 78.369 & 43 & 0.000 & 0.000 & 46.122 & \\
\hline 3 & $0.903(0.034)$ & $0.470(0.069)$ & $112.757(9.371)$ & 44 & $-0.299(0.276)$ & $-0.473(0.255)$ & ) 144.746 & (12.001) \\
\hline 4 & $0.941(0.031)$ & $0.754(0.060)$ & $29.177(2.465)$ & 45 & 0.000 & 0.000 & 15.325 & $(1.2$ \\
\hline 5 & 0.000 & 0.000 & $70.792(5.926)$ & 46 & 0.468 & 0.830 & ) 211.881 & $(17.568)$ \\
\hline 6 & $0.961(0.024)$ & $0.811(0.052)$ & $24.725(2.125)$ & 47 & 0.000 & 0.000 & 63.006 & $(5.227)$ \\
\hline 7 & $0.898(0.062)$ & $0.767(0.091)$ & $37.122(3.168)$ & 48 & 0.000 & 0.000 & 689.215 & $(57.149)$ \\
\hline 8 & 0.000 & .000 & 22.6 & 49 & $-0.424(0$ & $-0.203(0.239)$ & ) 32.114 & $(2.673$ \\
\hline 9 & 0.980 & 0.819 & 22.489 & 50 & -0.043 & 0.273 & 183.986 & $(15.264)$ \\
\hline 10 & $0.983(0.012)$ & 0.797 & $4.482(0.394)$ & 51 & & 0.000 & 20.391 & \\
\hline 11 & $0.978(0.014)$ & 0.761 & $8.523(0.725)$ & 52 & 0.9 & $0.811(0.0$ & 5.039 & $(0.422)$ \\
\hline 12 & 0.913 & 0.432 & 7.197 & 53 & 0.0 & $0.266(0.309)$ & 291.773 & $(24.221)$ \\
\hline 13 & 0.98 & 0.886 & 14.1 & 54 & 0.000 & 0.000 & 13.672 & 140) \\
\hline 14 & 0.025 & 0.220 & 80.586 & 55 & 0.968 & 0.715 & & \\
\hline 15 & $0.931(0.047)$ & 0.819 & 13.359 & 56 & & 0.811 & & \\
\hline 16 & $0.921(0.100)$ & 0.877 & $7.852(0.657)$ & 57 & 0. & 0.509 & 48. & $(4.027)$ \\
\hline 17 & 0.6 & 0.7 & 13. & 5 & & & & \\
\hline 18 & 0.000 & .000 & 53. & 59 & 0. & 0.518 & 91 & \\
\hline 19 & 0.000 & 0000 & 47.0 & 60 & 0. & $0.740(0$. & & \\
\hline 20 & 0.97 & 0.883 & $59.655(4.991)$ & 61 & 0. & 0.558 & 3.167 & $(0.263)$ \\
\hline 21 & $0.969(0$ & $0.898(0.0$ & $6.569(0.551)$ & 62 & & 0.000 & 10.871 & $(0.904)$ \\
\hline 22 & 0.00 & 0.000 & 3) & 63 & & 0.000 & 20. & $(1.74$ \\
\hline 23 & 0.000 & 0.000 & 60. & 64 & 57) & 0.832 & 158.608 & $(13.160)$ \\
\hline 24 & $0.937(0.046)$ & $0.841(0.071)$ & $4.965(\mathrm{c}$ & 65 & & 0.000 & 15. & $(1.431)$ \\
\hline 25 & $0.903(0.057)$ & $0.763(0.086)$ & $13.737(1.174)$ & 66 & 0. & 0.000 & 30.885 & $(2.562)$ \\
\hline 26 & 0.000 & 0.000 & $4(3.135)$ & 67 & 0. & 0.791 & & $(0.166)$ \\
\hline 27 & 0.93 & 0.6 & $0)$ & 68 & 0. & 0.619 & 1.084 & $(0.09$ \\
\hline 28 & 0.000 & 0.000 & 3) & 69 & 1 & $0.735(0.052)$ & 2.248 & $(0.186)$ \\
\hline 29 & $0.966(0.0$ & $0.758(0$. & $6.406(0.541)$ & 70 & & 0.000 & 26.847 & $(2.227)$ \\
\hline 30 & $0.908(0.033)$ & $0.485(0.070)$ & $3.934(0.356)$ & 71 & & 0.000 & 278.380 & $(23.109$ \\
\hline 31 & $0.970(0$ & 0.869 & $16.520(1$. & 72 & 0.0 & $0.183(0$ & 63.386 & $(5.257)$ \\
\hline 32 & 0.000 & 0.000 & $5(4.571)$ & 73 & 0.000 & 0.000 & 72.657 & $(6.031)$ \\
\hline 33 & $0.870(0.081)$ & $0.743(0.111)$ & $19.082(1.626)$ & 74 & $0.055(0.368)$ & $-0.107(0.366)$ & 17.384 & $(1.454)$ \\
\hline 34 & 0.000 & 0.000 & $5.503(0.457)$ & 75 & 0.000 & 0.000 & 7.423 & $(0.622)$ \\
\hline 35 & 0.0 & & & 76 & 8) & 0.665 & 10.737 & $(0.9$ \\
\hline 36 & 0.000 & 0.000 & $10.322(0.857)$ & 77 & 0.000 & 0.000 & 7.504 & $(0.62$ \\
\hline 37 & 0.000 & 0.000 & $37.501(3.148)$ & 78 & $0.879(0.048)$ & $0.604(0.080)$ & 6.007 & $(0.499)$ \\
\hline 38 & 0.000 & 0.000 & $93.621(7.783)$ & 79 & 0.000 & 0.000 & 525.057 & $(43.536)$ \\
\hline 39 & 0.000 & 0.000 & $29.886(2.481)$ & 80 & $0.942(0.035)$ & $0.803(0.061)$ & 3.619 & $(0.301)$ \\
\hline 40 & 0.000 & 0.000 & $22.070(1.841)$ & 81 & 0.000 & 0.000 & 5.515 & $(0.45$ \\
\hline 41 & 0.000 & 0.000 & $52.014(4.318)$ & & & & & \\
\hline
\end{tabular}




\title{
BANCO DE ESPAÑA PUBLICATIONS
}

\author{
WORKING PAPERS
}

1501 LAURA HOSPIDO and EVA MORENO-GALBIS: The Spanish productivity puzzle in the Great Recession.

1502 LAURA HOSPIDO, ERNESTO VILLANUEVA and GEMA ZAMARRO: Finance for all: the impact of financial literacy training in compulsory secondary education in Spain.

1503 MARIO IZQUIERDO, JUAN F. JIMENO and AITOR LACUESTA: Spain: from immigration to emigration?

1504 PAULINO FONT, MARIO IZQUIERDO and SERGIO PUENTE: Real wage responsiveness to unemployment in Spain: asymmetries along the business cycle.

1505 JUAN S. MORA-SANGUINETTI and NUNO GAROUPA: Litigation in Spain 2001-2010: Exploring the market for legal services.

1506 ANDRES ALMAZAN, ALFREDO MARTÍN-OLIVER and JESÚS SAURINA: Securitization and banks' capital structure.

1507 JUAN F. JIMENO, MARTA MARTÍNEZ-MATUTE and JUAN S. MORA-SANGUINETTI: Employment protection legislation and labor court activity in Spain.

1508 JOAN PAREDES, JAVIER J. PÉREZ and GABRIEL PEREZ-QUIRÓS: Fiscal targets. A guide to forecasters?

1509 MAXIMO CAMACHO and JAIME MARTINEZ-MARTIN: Monitoring the world business cycle.

1510 JAVIER MENCÍA and ENRIQUE SENTANA: Volatility-related exchange traded assets: an econometric investigation.

1511 PATRICIA GÓMEZ-GONZÁLEZ: Financial innovation in sovereign borrowing and public provision of liquidity.

1512 MIGUEL GARCÍA-POSADA and MARCOS MARCHETTI: The bank lending channel of unconventional monetary policy: the impact of the VLTROs on credit supply in Spain.

1513 JUAN DE LUCIO, RAÚL MÍNGUEZ, ASIER MINONDO and FRANCISCO REQUENA: Networks and the dynamics of firms' export portfolio.

1514 ALFREDO IBÁÑEZ: Default near-the-default-point: the value of and the distance to default.

1515 IVÁN KATARYNIUK and JAVIER VALLÉS: Fiscal consolidation after the Great Recession: the role of composition.

1516 PABLO HERNÁNDEZ DE COS and ENRIQUE MORAL-BENITO: On the predictability of narrative fiscal adjustments.

1517 GALO NUÑO and CARLOS THOMAS: Monetary policy and sovereign debt vulnerability.

1518 CRISTIANA BELU MANESCU and GALO NUÑO: Quantitative effects of the shale oil revolution.

1519 YAEL V. HOCHBERG, CARLOS J. SERRANO and ROSEMARIE H. ZIEDONIS: Patent collateral, investor commitment and the market for venture lending.

1520 TRINO-MANUEL ÑíGUEZ, IVAN PAYA, DAVID PEEL and JAVIER PEROTE: Higher-order risk preferences, constant relative risk aversion and the optimal portfolio allocation.

1521 LILIANA ROJAS-SUÁREZ and JOSÉ MARÍA SERENA: Changes in funding patterns by Latin American banking systems: how large? how risky?

1522 JUAN F. JIMENO: Long-lasting consequences of the European crisis.

1523 MAXIMO CAMACHO, DANILO LEIVA-LEON and GABRIEL PEREZ-QUIROS: Country shocks, monetary policy expectations and ECB decisions. A dynamic non-linear approach.

1524 JOSÉ MARÍA SERENA GARRALDA and GARIMA VASISHTHA: What drives bank-intermediated trade finance? Evidence from cross-country analysis.

1525 GABRIELE FIORENTINI, ALESSANDRO GALESI and ENRIQUE SENTANA: Fast ML estimation of dynamic bifactor models: an application to European inflation.

1526 YUNUS AKSOY and HENRIQUE S. BASSO: Securitization and asset prices.

1527 MARÍA DOLORES GADEA, ANA GÓMEZ-LOSCOS and GABRIEL PEREZ-QUIROS: The Great Moderation in historical perspective. Is it that great?

1528 YUNUS AKSOY, HENRIQUE S. BASSO, RON P. SMITH and TOBIAS GRASL: Demographic structure and macroeconomic trends.

1529 JOSÉ MARÍA CASADO, CRISTINA FERNÁNDEZ and JUAN F. JIMENO: Worker flows in the European Union during the Great Recession.

1530 CRISTINA FERNÁNDEZ and PILAR GARCÍA PEREA: The impact of the euro on euro area GDP per capita.

1531 IRMA ALONSO ÁLVAREZ: Institutional drivers of capital flows.

1532 PAUL EHLING, MICHAEL GALLMEYER, CHRISTIAN HEYERDAHL-LARSEN and PHILIPP ILLEDITSCH: Disagreement about inflation and the yield curve.

1533 GALO NUÑO and BENJAMIN MOLL: Controlling a distribution of heterogeneous agents. 
1534 TITO BOERI and JUAN F. JIMENO: The unbearable divergence of unemployment in Europe.

1535 OLYMPIA BOVER: Measuring expectations from household surveys: new results on subjective probabilities of future house prices.

1536 CRISTINA FERNÁNDEZ, AITOR LACUESTA, JOSÉ MANUEL MONTERO and ALBERTO URTASUN: Heterogeneity of markups at the firm level and changes during the great recession: the case of Spain.

1537 MIGUEL SARMIENTO and JORGE E. GALÁN: The influence of risk-taking on bank efficiency: evidence from Colombia.

1538 ISABEL ARGIMÓN, MICHEL DIETSCH and ÁNGEL ESTRADA: Prudential filters, portfolio composition and capital ratios in European banks.

1539 MARIA M. CAMPOS, DOMENICO DEPALO, EVANGELIA PAPAPETROU, JAVIER J. PÉREZ and ROBERTO RAMOS: Understanding the public sector pay gap.

1540 ÓSCAR ARCE, SAMUEL HURTADO and CARLOS THOMAS: Policy spillovers and synergies in a monetary union.

1601 CHRISTIAN CASTRO, ÁNGEL ESTRADA and JORGE MARTÍNEZ: The countercyclical capital buffer in Spain: an analysis of key guiding indicators.

1602 TRINO-MANUEL ÑÍGUEZ and JAVIER PEROTE: Multivariate moments expansion density: application of the dynamic equicorrelation model.

1603 ALBERTO FUERTES and JOSÉ MARÍA SERENA: How firms borrow in international bond markets: securities regulation and market segmentation.

1604 ENRIQUE ALBEROLA, IVÁN KATARYNIUK, ÁNGEL MELGUIZO and RENÉ OROZCO: Fiscal policy and the cycle in Latin America: the role of financing conditions and fiscal rules.

1605 ANA LAMO, ENRIQUE MORAL-BENITO and JAVIER J. PÉREZ: Does slack influence public and private labour market interactions?

1606 FRUCTUOSO BORRALLO, IGNACIO HERNANDO and JAVIER VALLÉS: The effects of US unconventional monetary policies in Latin America.

1607 VINCENZO MERELLA and DANIEL SANTABÁRBARA: Do the rich (really) consume higher-quality goods? Evidence from international trade data.

1608 CARMEN BROTO and MATÍAS LAMAS: Measuring market liquidity in US fixed income markets: a new synthetic indicator.

1609 MANUEL GARCÍA-SANTANA, ENRIQUE MORAL-BENITO, JOSEP PIJOAN-MAS and ROBERTO RAMOS: Growing like Spain: 1995-2007

1610 MIGUEL GARCÍA-POSADA and RAQUEL VEGAS: Las reformas de la Ley Concursal durante la Gran Recesión.

1611 LUNA AZAHARA ROMO GONZÁLEZ: The drivers of European banks' US dollar debt issuance: opportunistic funding in times of crisis?

1612 CELESTINO GIRÓN, MARTA MORANO, ENRIQUE M. QUILIS, DANIEL SANTABÁRBARA and CARLOS TORREGROSA: Modelling interest payments for macroeconomic assessment.

1613 ENRIQUE MORAL-BENITO: Growing by learning: firm-level evidence on the size-productivity nexus.

1614 JAIME MARTÍNEZ-MARTíN: Breaking down world trade elasticities: a panel ECM approach.

1615 ALESSANDRO GALESI and OMAR RACHEDI: Structural transformation, services deepening, and the transmission of monetary policy.

1616 BING XU, ADRIAN VAN RIXTEL and HONGLIN WANG: Do banks extract informational rents through collateral?

1617 MIHÁLY TAMÁS BORSI: Credit contractions and unemployment.

1618 MIHÁLY TAMÁS BORSI: Fiscal multipliers across the credit cycle.

1619 GABRIELE FIORENTINI, ALESSANDRO GALESI and ENRIQUE SENTANA: A spectral EM algorithm for dynamic factor models.

BANCODEESPAÑA

$$
\text { Eurosistema }
$$

Unidad de Servicios Auxiliares

Alcalá, 48 - 28014 Madrid

E-mail: publicaciones@bde.es www.bde.es 